TRANSACTIONS OF THE

AMERICAN MATHEMATICAL SOCIETY

Volume 365, Number 3, March 2013, Pages 1305-1345

S 0002-9947(2012)05600-5

Article electronically published on July 18, 2012

\title{
THE NONLOW COMPUTABLY ENUMERABLE DEGREES ARE NOT INVARIANT IN $\mathcal{E}$
}

\author{
RACHEL EPSTEIN
}

\begin{abstract}
We study the structure of the computably enumerable (c.e.) sets, which form a lattice $\mathcal{E}$ under set inclusion. The upward closed jump classes $\overline{\mathbf{L}}_{n}$ and $\mathbf{H}_{n}$ have all been shown to be definable by a lattice-theoretic formula, except for $\overline{\mathbf{L}}_{1}$, the nonlow degrees. We say a class of c.e. degrees is invariant if it is the set of degrees of a class of c.e. sets that is invariant under automorphisms of $\mathcal{E}$. All definable classes of degrees are invariant. We show that $\overline{\mathbf{L}}_{1}$ is not invariant, thus proving a 1996 conjecture of Harrington and Soare that the nonlow degrees are not definable, and completing the problem of determining the definability of each jump class. We prove this by constructing a nonlow c.e. set $D$ such that for all c.e. $A \leq_{\mathrm{T}} D$, there is a low set $B$ such that $A$ can be taken by an automorphism of $\mathcal{E}$ to $B$.
\end{abstract}

\section{INTRODUCTION}

Definability is one of the fundamental themes in computability theory. The problem of determining which classes of Turing degrees are definable in the languages of Turing reduction and set inclusion has long been a major topic of study among computability theorists. In particular, the question of which jump classes of computably enumerable (c.e.) degrees are definable in the language of set inclusion has been studied for over 40 years. We complete the answer to this question by showing that the class of nonlow c.e. degrees is not definable.

1.1. The main result. The computably enumerable (c.e.) degrees are a particularly important class of Turing degrees. They have been studied extensively since Post first asked in 1944 [10] whether there is a c.e. degree strictly between $\mathbf{0}$ and $\mathbf{0}^{\prime}$. Such degrees were found independently by Friedberg and Muchnik in the 1950s using the priority method, which we will use here. The c.e. sets can be equivalently defined as the domains of partial computable functions and as $\Sigma_{1}^{0}$ sets. The definition we use here is that a set $C$ is computably enumerable (c.e.) if there is a uniformly computable sequence of computable sets $\left\{C_{s}\right\}_{s \in \omega}$ such that $C=\bigcup_{s} C_{s}$. A degree is said to be c.e. if it contains a c.e. set.

Another important class of degrees below $\mathbf{0}^{\prime}$ are the low degrees. A degree $\mathbf{d}<\mathbf{0}^{\prime}$ is low (or $\mathbf{L}_{1}$ ) if $\mathbf{d}^{\prime}=\mathbf{0}^{\prime}$. The concept of lowness has been generalized to reflect the behavior of the $n^{\text {th }}$ jump of $\mathbf{d}$. In particular, a degree $\mathbf{d}$ is $\operatorname{low}_{\mathrm{n}}$ (or $\mathbf{L}_{n}$ ) if $\mathbf{d}^{(\mathbf{n})}=\mathbf{0}^{(\mathbf{n})}$. Similarly, a degree $\mathbf{d}$ is high $\left(\right.$ or $\left.\mathbf{H}_{1}\right)$ if $\mathbf{d}^{\prime}=\mathbf{0}^{\prime \prime}$ and is high ${ }_{\mathrm{n}}\left(\right.$ or $\left.\mathbf{H}_{n}\right)$ if

Received by the editors January 28, 2011 and, in revised form, April 8, 2011.

2010 Mathematics Subject Classification. Primary 03D25.

Key words and phrases. Computably enumerable, recursively enumerable, definability, automorphisms, invariance.

The author would like to thank Bob Soare for many helpful comments and conversations. 
$\mathbf{d}^{(\mathbf{n})}=\mathbf{0}^{(\mathbf{n}+\mathbf{1})}$. We call these classes and their complements jump classes of degrees. We will also sometimes call a set $\operatorname{low}_{n}$ or high $_{n}$ if it is of low or high $_{n}$ degree.

Among the jump classes, the low degrees are perhaps the most studied and most significant class. For instance, Gödel's incompleteness theorem tells us that there is no computable completion of Peano arithmetic. However, Peano arithmetic has a low completion. As we will see in \$1.3, all upward-closed jump classes except for the nonlow degrees have been shown to be definable in the lattice of c.e. sets $\mathcal{E}$ under set inclusion. We will show that the nonlow degrees are in fact not definable, setting them apart from all other jump classes.

Theorem 1.1 (Main theorem). The nonlow degrees are not definable in $\mathcal{E}$.

1.2. Methods of the proof. If we were to try to show that the nonlow degrees were definable in $\mathcal{E}$, we would look for some lattice-theoretic property $P$ such that $P(A)$ holds for some $A$ in every nonlow degree, but $P(A)$ does not hold for any low set $A$. We instead will show that the nonlow degrees are not definable, so we must show that there is no such property $P$. We can use automorphisms to achieve this.

Definition 1.2. A class of sets $\mathcal{S} \subseteq \mathcal{E}$ is invariant if it is closed under $\operatorname{Aut}(\mathcal{E})$, the set of all automorphisms of $\mathcal{E}$. A class of degrees $\mathcal{C}$ is invariant if

$$
\mathcal{C}=\{\operatorname{deg}(W) \mid W \in \mathcal{S}\}
$$

where $\mathcal{S}$ is invariant.

All classes of degrees definable in $\mathcal{E}$ are invariant because automorphisms preserve all lattice-theoretic properties. Thus, in order to show that a class is not definable, it suffices to show that it is not invariant.

To prove Theorem 1.1, we will show that the nonlow degrees are not invariant under automorphisms of $\mathcal{E}$. We actually prove something stronger. It suffices to show that all sets in a certain nonlow degree can be taken by automorphisms to low sets. However, we will show that all sets Turing reducible to a particular nonlow degree can be taken by automorphisms to low sets.

Theorem 1.3. There is a nonlow c.e. set $D$ such that for all c.e. $A \leq_{\mathrm{T}} D$, there exists a low c.e. set $B$ and an automorphism of $\mathcal{E}$ taking $A$ to $B$.

Theorem 1.1 follows because Theorem 1.3 shows that the nonlow degrees are not invariant.

One technique used in the proof is the Harrington-Soare automorphism method from [4. However, we will not be able to simply build an automorphism from $A$ to $B$ using only this method, as it does not allow for any restraint to be put on either set $A$ or $B$. Instead, we must break up the proof into two parts, performed simultaneously. The first is to build a partial automorphism on the complements of $A$ and $B$, and then to extend it to a full automorphism. The Extension Theorem from [13] does not work here, as we cannot get an effective automorphism. Instead, we modify the $\Delta_{3}^{0}$ automorphism method from [4] so that it can act as an extension theorem.

As we will see in $\$ 1.3$, our set $D$ must be $\operatorname{low}_{2}$. In $\$ 3$, we construct a nonlow c.e. set that is $\operatorname{low}_{2}$. While this theorem is already known, it illustrates the technique we will use to prove our main theorem. While we will not specifically make our set $D$ low $_{2}$, the fact that the construction succeeds guarantees that $D$ is $\operatorname{low}_{2}$. 
Both the Harrington-Soare $\Delta_{3}^{0}$ automorphism construction and the construction of a low 2 set that is nonlow are performed on trees of strategies. We will combine these trees into one tree that will provide the framework for building an automorphism from $A$ to $B$ while guaranteeing that $D$ is nonlow and $B$ is low. In order to combine these two methods, we must make significant changes to both. In 4 , we will discuss the methods of the proof in more detail before we begin the formal proof.

1.3. Historical background. We can examine the c.e. sets and degrees by looking at the structure $\mathcal{R}$ of the c.e. degrees under Turing reducibility or at the structure $\mathcal{E}$ of the c.e. sets under set inclusion. Notice that $\mathcal{E}$ forms a lattice with least element $\emptyset$ and greatest element $\omega$.

Definition 1.4. A class of sets $\mathcal{S}$ is definable in $\mathcal{E}$ if we can describe $\mathcal{S}$ in the language of set inclusion, and a class of sets $\mathcal{S}$ is definable in $\mathcal{R}$ if we can describe $\mathcal{S}$ in the language of Turing reducibility. A class of degrees $\mathbf{D}$ is definable in $\mathcal{E}$ or $\mathcal{R}$ if there is a class of sets $\mathcal{S}$ definable in $\mathcal{E}$ or $\mathcal{R}$ such that $\mathbf{D}=\{\operatorname{deg}(W) \mid W \in \mathcal{S}\}$.

It is natural to ask which jump classes of degrees are definable in the structures $\mathcal{E}$ and $\mathcal{R}$. We will discuss the problem for $\mathcal{E}$ and refer to $\mathcal{R}$ only for comparison. For the structure $\mathcal{E}$, the first progress on this problem came in the form of Martin's Theorem 1.5. We say a c.e. set $M$ is maximal if for every c.e. set $W_{e}$, either $W_{e} \subseteq^{*} M$ or $W_{e} \cup M=^{*} \omega$. The property of being a maximal set is latticetheoretic, or definable in $\mathcal{E}$.

Theorem 1.5 (Martin [8]). The high degrees $\mathbf{H}_{1}$ are exactly the degrees of the maximal sets.

Thus, the high degrees are definable in $\mathcal{E}$. This theorem marked the beginning of investigations as to which jump classes of degrees are definable in $\mathcal{E}$.

It is not difficult to see that the degree $\mathbf{0}$ is definable in $\mathcal{E}$, as it is the degree of the least element $\emptyset$ of the lattice $\mathcal{E}$. In addition, the set of all nonzero degrees is definable in $\mathcal{E}$ as the degrees of $\{A \in \mathcal{E} \mid \bar{A} \notin \mathcal{E}\}$. Harrington showed in 1986 (see Soare [15]) that the degree $\mathbf{0}^{\prime}$ is definable by finding a definition for the class of creative sets, which is a class of sets known to be complete.

A c.e. set $A$ is called atomless if $A$ is not contained in any maximal set. The atomless sets are clearly a class definable in $\mathcal{E}$.

Theorem 1.6 (Lachlan [6]). All atomless sets are nonlow $_{2}$.

Theorem 1.7 (Shoenfield [1]). Every nonlow 2 c.e. degree contains an atomless c.e. set.

This shows that the degrees of the atomless c.e. sets are the nonlow 2 degrees. Thus, the nonlow 2 c.e. degrees are definable in $\mathcal{E}$.

The first nondefinability results came in 1995, many years after the first definability results.

Theorem 1.8 (Cholak [1]; Harrington-Soare [4]). Every noncomputable c.e. set can be taken by an automorphism of $\mathcal{E}$ to a high set.

This theorem showed that the downward closed jump classes $\mathbf{L}_{n}, n>0$ and $\overline{\mathbf{H}}_{n}$, $n>0$ are not invariant and thus not definable. Harrington and Soare [4] used prompt sets to show that $\overline{\mathbf{H}}_{0}$, the class of incomplete c.e. degrees, is not definable. 
This completed the problem of showing the noninvariance of the noncomputable downward closed jump classes.

Definition 1.9. A c.e. set $A$ is prompt if there is an enumeration $\left\{A_{s}\right\}$ of $A$ and a computable function $p$ such that for all $s, p(s) \geq s$, and for all $e$,

$$
W_{e} \text { infinite } \Longrightarrow(\exists x)(\exists s)\left[x \in W _ { e , \text { at } s } \& A _ { s } \left\lceilx \neq A_{p(s)}\lceil x],\right.\right.
$$

where $C \uparrow x$ denotes the first $x$ bits of $C$.

Promptness is a property of degrees, as every set Turing equivalent to a prompt set is also prompt.

Theorem 1.10 (Harrington-Soare [4]). For all prompt sets $A$, there exists $B \in \mathbf{0}^{\prime}$ such that $A$ is automorphic to $B$.

It is well known that there is a low prompt degree $\mathbf{d}$, so every set $A \in \mathbf{d}$ can be taken by an automorphism of $\mathcal{E}$ to a complete set $B$. This reconfirms that the low degrees are not invariant, and neither is any other class of degrees that contains the low degrees and does not contain $\mathbf{0}^{\prime}$. Thus, every downward closed jump class $\mathbf{L}_{n}$ for $n \geq 1$ and $\overline{\mathbf{H}}_{n}$ for $n \geq 0$ is neither invariant nor definable in $\mathcal{E}$.

The only remaining jump classes in 1996 were the upward closed jump classes $\overline{\mathbf{L}}_{n}$ for $n=1$ and $n \geq 3$ and $\mathbf{H}_{n}$ for $n \geq 2$.

Theorem 1.11 (Cholak-Harrington [2]). For $n \geq 2, \mathbf{H}_{n}$ and $\overline{\mathbf{L}}_{n}$ are definable.

This is analogous to the result of Nies, Shore, and Slaman 9] for the c.e. degrees $\left(\mathcal{R},<_{T}\right)$ showing that $\mathbf{L}_{n}$ and $\mathbf{H}_{n}$ for $n \geq 2$ (and, equivalently, their complements) are definable in $\mathcal{R}$.

The only remaining case for the structure $\mathcal{E}$ is the class of nonlow degrees, $\overline{\mathbf{L}}_{1}$. We will show that this class differs from all the other upward closed jump classes, as it is the only one that is not definable. For the structure $\mathcal{R}$ of c.e. degrees under Turing reducibility, all jump classes are known to be definable except for the class of low degrees, which is still unknown. In both structures $\mathcal{E}$ and $\mathcal{R}$, the single jump is more difficult to define than the double jump.

\section{Definitions And NOtATion}

We give some basic definitions that will be used throughout this paper. Definitions specific to the automorphism construction will appear in $\$ 5$,

Let $C \subset \omega$. Then $C$ can be viewed as an element of $2^{\omega}$ by means of its characteristic function. For any set $C$, node $\alpha$, or path $f$, we write $C \uparrow n$ to denote the initial segment of $C$ of length $n$ and similarly for $\alpha\lceil n$ and $f\lceil n$.

Let $\Phi_{e}^{C}$ be the $e^{\text {th }}$ Turing functional with oracle $C$. We define the use $\varphi_{e}^{C}(n)$ of the computation $\Phi_{e}^{C}(n)$ by

$$
\varphi_{e}^{C}(n)= \begin{cases}m+1, & \text { if } \Phi_{e}^{C}(n) \downarrow, \text { where } m \text { is the largest bit of } C \\ \text { queried in the computation } \Phi_{e}^{C}(n) & \text { otherwise. }\end{cases}
$$

Let $C$ be a c.e. set with computable enumeration $C=\bigcup_{s \in \omega} C_{s}$. We write $\Phi_{e}^{C}(n)[s]$ to mean $\Phi_{e, s}^{C_{s}}(n)$, the result of the Turing functional $\Phi_{e}$ with oracle $C_{s}$ on input $n$, after $s$ steps of computation. Similarly, we write $\varphi_{e}^{C}(n)[s]$ for the use of that computation. 
Let $\langle x, y\rangle$ be the standard pairing function from $\omega^{2}$ to $\omega$. We will also use $\langle a, b, c\rangle=\langle\langle a, b\rangle, c\rangle$, allowing us to view sets as three-dimensional matrices, where each "row" of the matrix is itself a two-dimensional matrix.

A tree $T$ is a subset of $\omega^{<\omega}$ such that if $\alpha \in T$, then every initial segment of $\alpha$ is in $T$. Let $[T]$ be the set of infinite paths through $T$, where $h$ is an infinite path through $T$ if $h\lceil n \in T$ for all $n$. Let $\alpha, \beta, \gamma, \delta, \ldots$ range over $T$. Let $|\alpha|$ denote the length of $\alpha$. Let $\alpha \preceq \beta$ ( $\alpha \prec \beta$ ) denote that string $\beta$ extends (properly extends) $\alpha$. Let $\lambda$ denote the empty string, and $\alpha^{-}$denote the predecessor of $\alpha$ if $\alpha \neq \lambda$. Let $\alpha \widehat{\beta}$ denote the concatenation of string $\alpha$ followed by string $\beta$. In this paper, we work with trees in $\omega^{<\omega}$.

\section{A LOW 2 NONLOW C.E. SET $D$}

The existence of a low nonlow $_{1}$ c.e. set $D$ follows from the Sacks Jump Theorem [12] but that construction involves a two-stage process, one stage computable in $\mathbf{0}^{\prime}$, and is not useful in a computable construction for building automorphisms which we need here. Now we present another construction which will be key for our main theorem. It expands and corrects a sketch by Harrington and Soare [5], §5.2.

Theorem 3.1. There is a low $w_{2}$ nonlow c.e. set $D$.

3.1. Listing all $\Delta_{2}^{0}$ partial functions. Let $\left\{\widehat{\varphi}_{e}\right\}_{e \in \omega}$ be a listing of all $\Delta_{2}^{0}$ functions. That is, define $\widehat{\varphi}_{e}(x)=\lim _{z} \varphi_{e}(x, z)$ if the limit exists and if $\varphi_{e}$ is total, and $\widehat{\varphi}_{e}(x) \uparrow$ otherwise, where $\left\{\varphi_{e}\right\}_{e \in \omega}$ is the usual listing of all partial computable functions. It is useful to have a computable total function $\widetilde{\varphi}_{e, s}(x)$ which approximates the $\Delta_{2}^{0}$ function $\widehat{\varphi}_{e}(x)$ as follows.

Define $\widetilde{\varphi}_{e, s}(x)=\varphi_{e, s}(x, z)$, where $z$ is the maximum element such that $z \leq s$ and $\varphi_{e, s}(x, v) \downarrow$ for all $v \leq z$, with a default output of 0 if no such $z$ exists. Now the function $\widetilde{\varphi}$ is primitive recursive in the variables $e, s$, and $x$, and clearly

$$
\widehat{\varphi}_{e}(x)=y \quad \Longrightarrow \quad \lim _{s} \widetilde{\varphi}_{e, s}(x)=y \text {. }
$$

3.2. Making $D$ nonlow. To make $D$ nonlow we must diagonalize over all $\Delta_{2}^{0}$ functions which could make $D$ low.

We will meet for every $j$ the requirement,

$$
P_{j}: \quad \widehat{\varphi}_{j} \text { is not the characteristic function of }\left\{x: W_{x} \cap \bar{D} \neq \emptyset\right\} .
$$

This actually ensures the stronger property that $\bar{D}$ is not semi-low [15, p. 72], and hence that $D$ is not low.

The strategy for $P_{j}$. We define a set $Z_{j}$, whose index $g(j)$ is known a priori by the Recursion Theorem, so that $Z_{j}=W_{g(j)}$, where $g$ is a computable function. We describe the strategy in terms of a Lachlan game as in Lachlan [7] in which the Blue player (us) and the Red player (the opponent) simultaneously construct c.e. sets.

Step 1a. Initially, $Z_{j}=\emptyset$, and $Z_{j} \cap \bar{D}=\emptyset$. Wait until (if ever) the opponent recognizes this fact by producing a stage $t_{0}$ such that $\widetilde{\varphi}_{j, t_{0}}(g(j)) \downarrow=0$. Then choose a fresh element $\Gamma_{t_{0}}^{j}=x_{1}>x_{0}$ not yet in $D$, and put it in $Z_{j}$ so that (at least temporarily) $\bar{D} \cap Z_{j} \neq \emptyset$.

Step 1b. Wait until (if ever) the opponent produces a stage $t_{1}$ such that $\widetilde{\varphi}_{j, t_{1}}(g(j)) \downarrow$ $=1$. Then put $\Gamma_{t_{1}}^{j}=x_{1}$ into $D$ and repeat the procedure. (That is, go to Step $2 \mathrm{a}$ as above with a fresh element $x_{2}>x_{1}$ not yet in $D$.) 
3.2.1. The $\Sigma_{2}$-outcome for the $P_{j}$ strategy. The $\Sigma_{2}$-outcome is that this process is repeated finitely often. Then $\widetilde{\varphi}_{j, t}(g(j))$ gives the wrong answer for almost every $t$. Therefore, requirement $P_{j}$ is satisfied. Furthermore, $P_{j}$ appoints at most finitely many witnesses $\left\{x_{1}<x_{2}<\ldots<x_{n}\right\}$. This will cause only a finite set of injuries to the lower priority negative requirements described below.

3.2.2. The $\Pi_{2}$-outcome for the $P_{j}$ strategy. The $\Pi_{2}$-outcome is that this process is repeated infinitely often. Then $\lim _{t} \widetilde{\varphi}_{j, t}(g(j))[t]$ diverges. Therefore, requirement $P_{j}$ is satisfied because $\widehat{\varphi}_{j}(g(j))$ fails to give the correct value according to (11). In this case, the $P_{j}$ strategy enumerates an infinite set $\left\{x_{1}<x_{2}<\ldots\right\}$ of elements into $D$. However, this set is not only computable but is enumerated in strictly increasing order. Therefore, its effect on opposing negative requirements is easy to analyze.

Let $\Gamma_{s}^{j}$ or $\Gamma^{j}[s]$ denote the value of the witness $x_{n}$ for $P_{j}$ at the end of stage $s$ if it exists, and the maximum of $s$ and all previous witnesses otherwise. Choose the witnesses in $\omega^{[j]}$ in increasing order and such that $\Gamma_{s}^{j} \leq \Gamma_{s+1}^{j}$. We think of $\Gamma^{j}$ as a movable marker which is monotonically nondecreasing on a strictly increasing sequence of elements $\left\{x_{1}<x_{2}<\ldots\right\}$ and goes to infinity in the $\Pi_{2}$ case. Therefore, $\Gamma^{j}$ provides a lower bound for any future elements which will be contributed to $D$ by $P_{j}$.

3.3. Making $D \operatorname{low}_{2}$. Define $\operatorname{Tot}^{A}:=\left\{e: \Phi_{e}^{A}\right.$ is total $\}$ and Tot $:=\operatorname{Tot}^{\emptyset}$. Recall that $\operatorname{Tot}^{A} \equiv_{\mathrm{T}} A^{\prime \prime}$ and indeed $\operatorname{Tot}^{A}$ is $\Pi_{2}^{A}$-complete. The requirements must guarantee that

$$
\operatorname{Tot}^{X} \leq_{\mathrm{T}} \text { Tot }
$$

which implies that $X$ is low $_{2}$ by [15, Theorem IV.3.2 and Exer. IV.4.5]. Define

$$
\Psi_{e, s}^{X}(y)= \begin{cases}\Phi_{e, s}^{X}(y) & \text { if }(\forall z \leq y)\left[\Phi_{e, s}^{X}(z) \downarrow\right] \\ \text { undefined } & \text { otherwise. }\end{cases}
$$

Define the use function, $\psi_{e, s}(y):=\varphi_{e}^{D}(y)[s]$, also written $\psi_{e}(y)[s]$, if $\Psi_{e}^{D}(y)[s]$ is defined, and let $\psi_{e, s}(y)$ be undefined otherwise. Note that $\Psi_{e}^{X}$ is either total or has finite domain, and $\Psi_{e}^{X}$ is total if and only if $\Phi_{e}^{X}$ is total. The first outcome with $X=D$ is the $\Pi_{2}$-outcome for $\Psi_{e}^{D}$ and the second is the $\Sigma_{2}$-outcome. To achieve condition (3) we define the following pseudo-requirements:

$$
N_{e, y}: \quad\left(\exists^{\infty} s\right)\left[\Psi_{e}^{D}(y)[s] \downarrow\right] \quad \Longleftrightarrow \quad \Psi_{e}^{D}(y) \downarrow .
$$

Let $N_{e}$ be the conjunction, $N_{e}=\&\left\{N_{e, y}: y \in \omega\right\}$. Of course, we cannot literally satisfy these negative pseudo-requirements $N_{e, y}$ for every $e$ and $y$ or else $D$ would be low because they are exactly the lowness requirements of the low simple set construction in [15, p. 111]. Instead these $N_{e, y}$ are like the pseudo-requirements $Q_{e}$ in the proof of the Sacks Jump Theorem in [15. p. 138]. Attempting to satisfy them causes us to put on a restraint to preserve a computation $\psi(y)[s]$ whenever possible. This restraint may be injured infinitely often (but only computably) by a higher priority positive requirement and $\mathbf{0}^{\prime \prime}$ can discover that injury and can calculate when computations in (5) will no longer be injured and whether $\Psi_{e}^{D}(y)$ converges or not. Therefore, we use (5) to achieve the following:

$$
(\forall y)\left(\exists^{\infty} s\right)\left[\Psi_{e}^{D}(y)[s] \downarrow\right] \quad \Longleftrightarrow \quad \Psi_{e}^{D} \text { is total. }
$$


The left hand side is $\Pi_{2}$ and therefore computable in $\mathbf{0}^{\prime \prime}$. The right hand side is $\Pi_{2}^{D}$-complete. Therefore, $D^{\prime \prime} \leq_{\mathrm{T}} \mathbf{0}^{\prime \prime}$ and $D$ is low 2 .

3.4. The interference between requirements. We now consider the interference between opposing positive and negative requirements.

3.4.1. One $P_{j}$ versus one $N_{e}$ with $j<e$. Consider one positive requirement $P_{j}$ and one negative requirement $N_{e}$ where $j<e$ so that $P_{j}$ has higher priority. Our strategy for $N_{e}$ will be split into two parts, one in case $P_{j}$ has the $\Pi_{2}$-outcome and one in case $P_{j}$ has the $\Sigma_{2}$-outcome. We say that $N_{e}$ is injured by $P_{j}$ whenever the strategy for $P_{j}$ enumerates a witness $x_{n}<\psi_{e}(y)[s]$ into $D$.

The $\Sigma_{2}$-outcome for $P_{j}$. If $P_{j}$ has the $\Sigma_{2}$ outcome and $N_{e}$ is guessing correctly at the number of witnesses it will enumerate in $D$, then $N_{e}$ can wait until those witnesses have all been enumerated in $D$ before starting its strategy.

The $\Pi_{2}$-outcome for $P_{j}$. Suppose $P_{j}$ has the $\Pi_{2}$-outcome and $N_{e}$ is guessing this correctly. Then $N_{e}$ knows that the marker $\Gamma_{j}$ for $P_{j}$ is moving monotonically to infinity. $\quad N_{e}$ can replace the raw use function $\psi_{e}(y)$ by a modified use function $\widehat{\psi}_{e}(y)$ which is $\psi_{e}(y)$ whenever $\psi_{e}(y)[s]<\Gamma^{j}[s]$ and undefined otherwise. Any real computation with use $\psi_{e}(y)$ will eventually have this use below $\Gamma_{j}$ and therefore it will also equal $\widehat{\psi}_{e}(y)$. However, $P_{j}$ will never cause a witness $x<\widehat{\psi}_{e}(y)$ to enter $D$, so there will never be any injury to the function $\widehat{\psi}_{e}(y)$.

If there are two positive requirements of higher priority, $P_{j}$ and $P_{k}$ for $j, k<e$, then as the upper boundary for $N_{e}$ we take the minimum of $\Gamma^{j}[s]$ and $\Gamma^{k}[s]$. Again there will be no injury to $\widehat{\psi}_{e}(y)$. This is formalized for the general case in (7) and (8).

\subsubsection{One $N_{e}$ versus one $P_{j}$ with $e<j$.}

The $\Sigma_{2}$-outcome for $N_{e}$. In the $\Sigma_{2}$-outcome for $N_{e}$ there is some least $y$ such that $\Psi^{D}(y)$ is undefined. Therefore, by the definition of $\Psi_{e}$ and (5) there will be at most finitely many stages when a new computation $\psi_{e}(z)$ appears for any $z$ and only finitely many times $N_{e}$ wants to impose a new restraint. Whenever this happens, we restart the $P_{j}$ strategy which is therefore restarted (and injured) at most finitely often.

The $\Pi_{2}$-outcome for $N_{e}$. Even though $N_{e}$ has a higher priority than $P_{j}$ it must guarantee (5) for all $y$ not just for $y=e$. Therefore, $N_{e}$ cannot simply preserve $\psi_{e}(y)$ with priority $N_{e}$ against all followers $x_{n}$ for $P_{j}$ as in the construction of a low simple set.

The solution is to use the natural $\omega$ ranking of all negative pairs $\langle e, y\rangle$ against the $\omega$ ranking $\langle j, n\rangle$ of all positive pairs. $P_{j}$ guesses that $\widehat{\psi}_{e}(y)$ will eventually be defined for all $y$. When $P_{j}$ is ready to define a new $x_{n}$ it can wait until $\widehat{\psi}_{e}(y)[s]$ is defined for all $y$ with $\langle e, y\rangle\left\langle\langle j, n\rangle\right.$ and can then appoint $x_{n}>\psi_{e}(y)[s]$ for such a $y$.

Note that the computation $\psi_{e}(y)[s]$ cannot be destroyed at any stage $t>s$ because the witnesses $x_{k}, k<n$, have already entered $D$ by stage $s$ and $x_{n}$ the first active witness after $s$ exceeds the use. Of course, a computation $\widehat{\psi}_{e}(z)$ may be injured at some stage $t>s$ because it may have become defined after $x_{n}$ was appointed. However, $\widehat{\psi}_{e}(z)$ can be injured by only finitely many witnesses $x_{k}$ and at most once by each. Therefore, the subrequirement $N_{e, y}$ of (5) is met for every $y$. 
But when $P_{j}$ is ready to appoint $x_{n}$ it need only wait for $\widehat{\psi}_{e}(y)$ to be defined for all $\langle e, y\rangle\left\langle\langle j, n\rangle\right.$ which must eventually occur. The fact that $x_{n}$ is ready to be appointed means that all witnesses $x_{k}, k<n$, have already entered $D$. Therefore, these computations cannot later change and $x_{n}$ can carry out its strategy as before.

Notice that this argument succeeds only because when we define $x_{n}>\widehat{\psi}_{e}(y)[s]$ we know that $\widehat{\psi}_{e}(y)[s]$ cannot later change and hence that $x_{n}$ can never injure this computation. If we have two or more positive requirements we have to worry that another positive requirement may contribute a follower $z<\widehat{\psi}_{e}(y)[s]$ to $D$ at some later stage $t>s$ which allows $\widehat{\psi}_{e}(y)$ to be redefined so that $x_{n}<\widehat{\psi}_{e}(y)[t]$. This threatens to allow $x_{n}$ to later enter $D$ and injure the $\widehat{\psi}_{e}(y)$ computation. We address this obstacle in 33.7

3.5. A tree argument. To complete the proof we convert these strategies into a tree construction as described in [15, Chapter XIV]. Put the empty node $\lambda$ on $T$. If $\alpha \in T$ and $|\alpha|=2 e$, then we call $\alpha$ "even" and we associate with $\alpha$ a version of the strategy $N_{e}$, and put the nodes $\alpha^{\wedge} 0$ and $\widehat{\alpha} 1$ on $T$ which represent the $\Pi_{2}$ and $\Sigma_{2}$-outcomes, respectively, of the $\alpha$ strategy. If $\beta \in T$ and $|\beta|=2 i+1$, then we call $\beta$ "odd" and we associate with $\beta$ a version of the strategy for $P_{i}$, and put the outcomes $\beta^{\wedge} 0$ and $\beta^{\wedge} p$ for all $p \geq 1$ on $T$, representing the $\Pi_{2}$ and all possible $\Sigma_{2}$-outcomes of the $\beta$ strategy $\left(\beta^{\wedge} p\right.$ means a guess of exactly $p-1 \beta$-witnesses enumerated into $D$ ). The order of the successor nodes to $\beta$ will be $\beta$ - 0 on the left, and the others on the right in the order of $\omega^{*}$. The true path $f$ on $T$ and its computable approximation $\left\{f_{s}\right\}_{s \in \omega}$ such that $f=\liminf _{s} f_{s}$ will be defined below.

Suppose $\beta \in T$ and $|\beta|=2 j+1$. Then $\beta$ has an associated witness function $\Gamma_{s}^{\beta}$ for carrying out its strategy for $P_{j}$ as above. At each stage $s$ there is at most one such witness $\Gamma_{s}^{\beta}=x_{n}^{\beta}$, the $n^{\text {th }}$ witness appointed by $\beta$, and it can contribute at most that witness to $D$ at stage $s+1$. We arrange that $\Gamma_{s}^{\beta} \leq \Gamma_{s+1}^{\beta}$. (The following is the general case of the explanation in $\$ 3.4 .1$ in the paragraph on the $\Pi_{2}$-outcome for $P_{j}$.)

Suppose $\alpha \in T$ and $|\alpha|=2 e$. Define

(8) $\widehat{\psi}_{\alpha, s}(y)=\left\{\begin{array}{c}\psi_{e, s}(y) \quad \text { if } \psi_{e, s}(y) \downarrow<\Lambda_{s}^{\alpha} \text { and (for all odd } \beta \text { ) } \\ {[\beta \preceq p \preceq \alpha \Longrightarrow \text { all } p-1 \beta \text {-witnesses have entered } D]} \\ \text { undefined otherwise. }\end{array}\right.$

Note that $\lim _{s} \Lambda_{s}^{\alpha}=\infty$ if $\alpha \prec f$. For each $\beta \prec \alpha,|\beta|=2 i+1, \beta$ may put elements into $D$ without respecting the computations $\psi_{\alpha, s}(y)$ which $\alpha$ is trying to protect. Assume $\alpha \prec f$. Then no such $\beta$ will ever contribute to $D$ an element $x \leq \widehat{\psi}_{\alpha, s}(y)$. Choose a stage $s_{0}$ such that for each $\beta \prec \alpha,|\beta|=2 i+1$, and if $\beta \prec p \preceq \alpha$ for $p \neq 0$, or $\beta<_{L} \alpha$, then $\beta$ does not contribute an element to $D$ at any stage $s \geq s_{0}$. Now no $\beta<\alpha$ will contribute an element $x \leq \widehat{\psi}_{\alpha, s}(y)$ at any stage $s>s_{0}$.

But for odd length $\beta$ of lower priority $\beta \succeq \alpha^{\wedge} 0$, the $\beta$ witnesses are arranged as for $N_{e}$ in 33.3 . That is, such a $\beta$ can appoint the $n^{\text {th }}$ witness, $x_{n}^{\beta}$, only after $\widehat{\psi}_{\alpha, s}(y) \downarrow$ for all $y,\langle\alpha, y\rangle \leq\langle\beta, n\rangle$, and must exceed their values. (Here, we identify the nodes of $T$ with their Gödel numbering under some fixed effective numbering of $T$.) As discussed in 3.7, this is not quite as simple as it sounds. However, as we will see, for each $y$ the computation $\widehat{\psi}_{\alpha, s}(y)$ can be injured at most finitely often, 
giving

$$
N_{\alpha}: \quad(\forall y)\left[\left(\exists^{\infty} s\right)\left[\widehat{\psi}_{\alpha}^{D}(y)[s] \downarrow\right] \quad \Longleftrightarrow \quad \widehat{\psi}_{\alpha}^{D}(y) \downarrow\right] .
$$

Finally, $\operatorname{Tot}^{A} \leq_{\mathrm{T}} \mathbf{0}^{\prime \prime}$ because to determine whether $\Phi_{e}^{D}$ is total, we use $\mathbf{0}^{\prime \prime}$ to compute the node on the true path $\alpha=f\left\lceil 2 e\right.$. Now by $N_{\alpha}$ we know that $\Psi_{e}$ is total if and only if $(\forall y)\left(\exists^{\infty} s\right)\left[\widehat{\psi}_{\alpha}^{D}(y)[s] \downarrow\right]$, which is a $\Pi_{2}$ statement.

The ordering of the nodes on the tree is important. Note that the positive requirement nodes follow a different ordering, in that the 0 node is on the left, and the rest of the nodes are in the order of $\omega^{*}$.

Definition 3.2. Let $\alpha, \beta \in T$.

(i) Unless otherwise specified, $\alpha$ is to the left of $\beta\left(\alpha<_{L} \beta\right)$ if

$$
(\exists a, b \in \omega)(\exists \gamma \in T)\left[\gamma^{\wedge} a \preceq \alpha \quad \& \quad \widehat{\gamma} b \preceq \beta \quad \& a<b\right] .
$$

(ii) If $h \in[T]$ we say $\alpha<_{L} h$ if there exists $\beta \prec h$ such that $\alpha<_{L} \beta$ and similarly for $h<_{L} \alpha$.

3.6. The construction. Fix $T=2^{<\omega}$. Simultaneously with the construction of $D$ at each stage we define a function $f_{s} \in T,\left|f_{s}\right|=2 s$, such that $f=\liminf f_{s} f_{s}$. We say that stage $s$ is an $\alpha$-stage if $\alpha \prec f_{s}$. For each even $\alpha \in T$ define the function

$$
l(\alpha, s)=\max \left\{x:(\forall y<x)\left[\widehat{\psi}_{\alpha}^{D}(y)[s] \downarrow\right]\right\} .
$$

Stage $s=0$. Define $f_{0}=\lambda$ to be the empty node on $T$.

Stage $s+1$. Define $f_{s+1}$ by a series of substages $t, 0 \leq t \leq 2 s$, where we define $\delta^{t}$ at substage $t$, as follows, and then define $f_{s+1}=\delta^{2 s}$.

Substage $t, 0 \leq t \leq 2 s$. Define $\delta^{0}=\lambda$. Given $\delta^{t}$, perform the following action.

Case 1. $\left|\delta^{t}\right|$ is even. Let $\alpha=\delta^{t}$. Let $v$ be the last $\alpha$-stage less than $s$. If $l(\alpha, v)<$ $l(\alpha, s)$, then define $\delta^{t+1}=\delta^{t \curlywedge} 0$, and otherwise let $\delta^{t+1}=\delta^{t \frown} 1$.

Case 2. $\left|\delta^{t}\right|=2 j+1$. Let $\beta=\delta^{t}$. Here $\beta$ is associated with the strategy for $P_{j}$ described in 3.2 (In particular, we assume a set $Z_{\beta}$ is being defined whose index $g(\beta)$ is known a priori, such that $W_{g(\beta)}=Z_{\beta}, g$ is the computable function as in 3.2 Step 1a but adjusted with $\beta$ in place of $j$, and that our objective is to arrange that either $\lim _{s} \widetilde{\varphi}_{j, s}(g(\beta))$ diverges, or gives the wrong answer about whether $W_{g(\beta)} \cap \bar{D} \neq \emptyset$.)

Subcase 1. (Like $\oint 3.2$ Step 1a) Suppose $Z_{\beta, s} \cap \bar{D}_{s}=\emptyset, \widetilde{\varphi}_{j, s}(g(\beta)) \downarrow=0$, and there is no $\beta$-witness in $\bar{D}_{s}$. Let the $\beta$-witnesses previously appointed be $x_{1}^{\beta} \ldots x_{n-1}^{\beta}$. Suppose

$$
\begin{aligned}
& (\forall \text { even } \alpha)\left[\alpha \prec 0 \prec \beta \quad \Longrightarrow \quad(\forall p)\left[\langle\alpha, p\rangle \leq\langle\beta, n\rangle \Longrightarrow \widehat{\psi}_{\alpha, s}(p) \downarrow\right]\right] \text {, and } \\
& (\forall \text { odd } \xi)\left[\begin{array}{c}
\xi^{\prec} p \prec \beta \Longrightarrow x_{n^{\prime}}^{\xi} \in D_{s} \text { for all } n^{\prime}<p, \\
\text { and }\left(\xi^{\wedge} 0 \prec \beta \&\left\langle\xi, n^{\prime}\right\rangle<\langle\beta, n\rangle\right) \Longrightarrow x_{n^{\prime}}^{\xi} \in D_{s}
\end{array}\right] \text {. }
\end{aligned}
$$

Define $y$ to be the smallest $z \in \bar{D}_{s} \cap \omega^{[\beta]}$, where $\beta$ is identified with its Gödel number, such that $\Gamma_{s}^{\beta}<z<\Lambda_{s}^{\beta}$, and the following holds:

$$
\begin{gathered}
(\forall \text { even } \alpha)\left[\widehat{\alpha} 0 \prec \beta \Longrightarrow(\forall\langle\alpha, p\rangle<\langle\beta, n\rangle)\left[\widehat{\psi}_{\alpha, s}(p) \downarrow<z\right]\right] \\
\text { and }\left(\forall \text { odd } \gamma<_{L} \beta\right)\left[z>\Gamma_{s}^{\gamma}\right] .
\end{gathered}
$$


The above equation says that we choose a new $\beta$-witness $y$ to be bigger than the last $\beta$-witness and all $\gamma$-witnesses to the left of $\beta$, smaller than the higher priority witnesses, and that preserves higher priority restraint functions for the negative requirements. If $y$ exists, then appoint $y$ as the next $\beta$-witness, call it $x_{n}^{\beta}$, define $\Gamma_{s+1}^{\beta}=x_{n}^{\beta}$, and enumerate $x_{n}^{\beta}$ in $Z_{\beta, s+1}$.

Subcase 2. (Like $\$ 3.2$ Step 1b) Suppose that $\widetilde{\varphi}_{j, s}(g(\beta)) \downarrow=1, \Gamma_{s}^{\beta}$ is defined and equal to some $\beta$-witness $x_{n}^{\beta}$, and that

$$
(\forall \text { odd } \xi)\left[\xi^{\prec} 0 \prec \beta \quad \Longrightarrow \quad \Gamma_{s}^{\xi}=x_{p}^{\xi} \in D_{s+1}\right] .
$$

Then enumerate $x_{n}^{\beta}$ in $D_{s+1}$, and define

$$
\Gamma_{s+1}^{\beta}=\max \left\{\Gamma_{s}^{\beta}, s+1\right\} .
$$

(Equation (14) says that for every odd $\xi$ with $\xi^{\wedge} 0 \prec \beta$, i.e., for which $\beta$ is guessing that $\xi$ has the $\Pi_{2}$ outcome of infinitely many witnesses, $\beta$ must wait until $\xi$ actually puts its witness $\Gamma_{s}=x_{p}^{\xi}$ into $D_{s+1}$ during an earlier substage of stage $s+1$ before $\beta$ is allowed to simultaneously contribute its own witness at a later substage of stage $s+1$.)

Subcase 3. Otherwise, if $\Gamma_{s}^{\beta}=x_{n}^{\beta}$ for some $\beta$-witness $x_{n}^{\beta}$, then define $\Gamma_{s+1}^{\beta}=\Gamma_{s}^{\beta}$, and if not, then define $\Gamma_{s+1}^{\beta}=\max \left\{\Gamma_{s}^{\beta}, s+1\right\}$.

To complete Case 3 define $\delta^{t+1}=\delta^{t \wedge} 0$ if Subcase 2 holds, and otherwise $\delta^{t+1}=$ $\delta^{t \frown} p+1$, where $p$ is the number of elements $\beta$ has enumerated into $D$. Complete the construction by defining $f_{s+1}=\delta^{2 s}$ and by initializing every odd node $\beta$ such that $f_{s+1}<_{L} \beta$, that is, by canceling any $\beta$-witness, and defining $\Gamma_{s+1}^{\beta}=\max \left\{\Gamma_{s}^{\beta}, s+1\right\}$.

3.7. The obstacle. Before we begin the formal verification, we will discuss the primary obstacle in the proof, which was missing from the proof given in [5]. The obstacle appears in proving that along the true path, requirement $N_{\alpha}$ in (9) is met.

The tricky part is showing that for a node $\beta \succ \alpha$ with witness $x_{n}^{\beta}$, where $\langle\beta, n\rangle>\langle\alpha, y\rangle$, that $x_{n}^{\beta}$ cannot injure $\widehat{\psi}_{\alpha}^{D}(y)[t]$ for any $t$. Now, when $x_{n}^{\beta}$ was appointed as a witness at some stage $s$, it must have been chosen to respect the $\langle\alpha, y\rangle$ computation $\widehat{\psi}_{\alpha}^{D}(y)[s]$. The question is whether $x_{n}^{\beta}$ still respects the $\langle\alpha, y\rangle$ computation $\widehat{\psi}_{\alpha}^{D}(y)[t]$ at any stage $t>s$. If it does not, then $x_{n}^{\beta}$ could injure the $\langle\alpha, y\rangle$ computation when $x_{n}^{\beta}$ enters $D$.

This raises a new question: is it possible for $x_{n}^{\beta}$ to be defined at stage $s$, when the $\langle\alpha, y\rangle$ computation was less than $x_{n}^{\beta}$, and then enter $D$ at stage $t>s$, when the $\langle\alpha, y\rangle$ computation is greater than $x_{n}^{\beta}$ ? Note that the only way for this to happen is if something injures the $\langle\alpha, y\rangle$ computation between stages $s$ and $t$. In the verification, we will show by examining each type of node that it is not possible for this injury to occur. Thus, $x_{n}^{\beta}$ cannot injure $\widehat{\psi}_{\alpha}^{D}(y)[t]$ for any $t>s$.

3.8. The verification. We now verify that this construction establishes Theorem 3.1 First define the true path $f$ of the construction by induction on $e$. Let $f \uparrow$ $0=\lambda$, the empty node. Given $\alpha=f\left\lceil 2 e\right.$ define $f(2 e)=0$ if $(\forall y)\left[\left(\exists^{\infty} s\right)\left[\widehat{\psi}_{\alpha}^{D}(y)[s] \downarrow\right]\right.$, and $f(2 e)=1$ otherwise. Given $\beta=f \uparrow(2 e+1)$ define $f(2 e+1)=0$ if $\beta$ appoints infinitely many $\beta$-witnesses and $f(2 e+1)=p+1$ if $\beta$ appoints $p \beta$-witnesses that eventually enter $D$. 
Lemma 3.3. Fix $e \in \omega$. Let $\alpha=f\lceil 2 e$. Then the construction has satisfied the requirement,

$$
N_{\alpha}: \quad(\forall y)\left[\left(\exists^{\infty} s\right)\left[\widehat{\psi}_{\alpha}^{D}(y)[s] \downarrow\right] \quad \Longleftrightarrow \quad \widehat{\psi}_{\alpha}^{D}(y) \downarrow\right],
$$

so that $D$ is low .

Proof. Fix $\alpha=f\lceil 2 e$ and fix $y \in \omega$. The right to left direction is obvious. For the other direction, assume $\left(\exists^{\infty} s\right)\left[\widehat{\psi}_{\alpha}^{D}(y)[s] \downarrow\right]$. Assume by induction that

$$
(\exists t)(\forall s>t)(\forall z<y)\left[\widehat{\psi}_{\alpha}^{D}(z)[s] \downarrow=\widehat{\psi}_{\alpha}^{D}(z)\right] .
$$

Consider all the finitely many pairs $\langle\beta, n\rangle\langle\langle\alpha, y\rangle$. Let $p$ be the maximum of

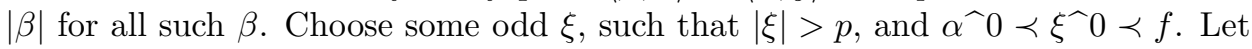
$t$ be as in (17). Choose $s_{0}>t$ such that for all $s \geq s_{0}$ : (i) if $\langle\beta, n\rangle\langle\langle\alpha, y\rangle$, then the $\beta$-witness $x_{n}^{\beta}$ does not become newly defined or enter $D$ at stage $s$; (ii) $f_{s} \nless_{L} \xi$ (so every odd $\beta<_{L} \xi$ has ceased to act by stage $s$ ); and (iii) every $\beta$ with $\beta^{\prec} p \prec \xi$, $p \neq 0$, has ceased to act by stage $s$. Thus, by choice of $s_{0}$ no witness $x_{n}^{\beta}$, for $\beta<_{L} \xi$, $\beta \prec p \prec \xi$, or $\langle\beta, n\rangle<\langle\alpha, y\rangle$ will ever contribute a $\beta$-witness to $D$ after stage $s_{0}$. In other words, these three types of witnesses cannot injure the $\langle\alpha, y\rangle$ computation after stage $s_{0}$.

Choose $s_{1} \geq s_{0}$ such that $\xi$ contributes a $\xi$-witness $x_{m}^{\xi}$ to $D$ at $s_{1}$. This action of $\xi$ causes initialization of all $\beta, \xi<_{L} \beta$, and cancellation of all such $\beta$-witnesses at stage $s_{1}$. By (14), all $\beta$ with $\beta^{\prec} 0 \prec \xi$ must have contributed their witnesses to $D$ by stage $s_{1}$, and so have no $\beta$-witnesses in existence at the end of stage $s_{1}$. Thus, for all $\beta$ with $\xi<_{L} \beta$ or $\beta \prec 0 \prec \xi$, no $\beta$-witnesses currently in existence at stage $s_{1}$ will ever injure the $\langle\alpha, y\rangle$ computation.

The above arguments show that for any $\beta \prec \xi$ or to the right or left of $\xi$, at stage $s_{1}, \beta$ has no witness that could possibly injure the computation $\widehat{\psi}_{\alpha, s_{1}}(y)$. We have also shown that if $\langle\beta, n\rangle<\langle\alpha, y\rangle$, then $x_{n}^{\beta}$ will not injure the $\langle\alpha, y\rangle$ computation. The only remaining case is when $\xi \prec \beta$ and $\langle\beta, n\rangle>\langle\alpha, y\rangle$. This is the case discussed in 93.7 . We describe it here in detail.

Consider $\xi \prec \beta$. Suppose $\langle\beta, n\rangle>\langle\alpha, y\rangle$ and $x_{n}^{\beta}$ is a witness that exists at stage $s_{1}$. If $x_{n}^{\beta}$ was appointed when $\widehat{\psi}_{\alpha, t}(y)$ was the same as at stage $s_{1}$, then $x_{n}^{\beta}$ must be larger than $\widehat{\psi}_{\alpha, s_{1}}(y)$, so it will not injure it. However, if $x_{n}^{\beta}$ was appointed when $\widehat{\psi}_{\alpha, t}(y)$ was smaller than it is at stage $s_{1}$, then at some stage after $s_{1}, x_{n}^{\beta}$ could injure the current computation. Now, the only way for this to happen is for something else to injure the computation before stage $s_{1}$ and after $x_{n}^{\beta}$ has been appointed. The injury could not come from a node $\gamma<_{L} \beta$ because that would reset the $\beta$-witness. It could not come from a node $\gamma \succ \beta$ because by (14), $\gamma$ must wait for $\beta$ to enumerate its witness before enumerating its own. Suppose the injury came from a node $\gamma$ to the right of $\beta$. Then to avoid being reset, $x_{n}^{\beta}$ must be defined before the $\gamma$-witness. Thus, by (13), $\gamma$ must have its witness larger than $x_{n}^{\beta}$, which means that $\gamma$ could not have injured the $\langle\alpha, y\rangle$ computation.

Now, if $\widehat{\gamma^{\gamma} p} \beta$ for any $p \neq 0$, then by (12),$\beta$ must wait for all $\gamma$-witnesses to enter $D$ before defining its own witness. If $\widehat{\gamma} 0 \prec \beta$, then $\beta$ must choose its witness smaller than the $\gamma$-witness, so $\gamma$ cannot injure the $\langle\alpha, y\rangle$ computation. Thus, no $\beta$-witness for $\xi \prec \beta$ appointed before stage $s_{1}$ can possibly injure $\widehat{\psi}_{\alpha, s_{1}}(y)$. 
Hence, at the end of stage $s_{1}$, the only $\beta$-witnesses in existence which have the priority to injure a computation $\widehat{\psi}_{\alpha, t}(y)$ for some $t \geq s_{1}$ are those which are permanently dormant and will never enter $D$.

Consider any new $\beta$-witness $x_{n}^{\beta}$ appointed after stage $s_{1}$. We cannot have $\beta<_{L} \xi$

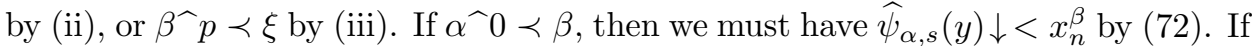
$f<_{L} \beta$, then $x_{n}^{\beta}$ must exceed $\widehat{\psi}_{\alpha, s}(y)$ if the latter converges when $x_{n}^{\beta}$ is appointed, and if not, $x_{n}^{\beta}$ must be canceled whenever it later converges. (Note that any $x_{n}^{\beta}$ newly appointed at $s$ must exceed $\widehat{\psi}_{\alpha}^{D}(y)[s]$ because the former must exceed $s$ by the definition of $\Gamma_{s}^{\beta}$, and the latter must be less than $s$ by the usual convention on the use function.) Choose $k>s_{1}$ such that $\widehat{\psi}_{\alpha, k}(y) \downarrow$. Then by induction on $v$ we have for all $v \geq k$ that $\widehat{\psi}_{\alpha, v}(y) \downarrow=\widehat{\psi}_{\alpha, k}(y) \downarrow=\widehat{\psi}_{\alpha}(y)$, and no new witness is appointed less than this value. Hence, $(\exists k)(\forall s>k)\left[\widehat{\psi}_{\alpha}^{D}(y)[s] \downarrow\right]$. By the discussion in $\$ 3.3 . D$ is $\operatorname{low}_{2}$.

Lemma 3.4. For every $j \in \omega$ the strategy of node $\beta=f \uparrow(2 j+1)$ has satisfied the requirement,

$$
P_{j}: \quad \widehat{\varphi}_{j} \neq \text { characteristic function of }\left\{x: W_{x} \cap \bar{D} \neq \emptyset\right\},
$$

so that $D$ is not low .

Proof. Fix $\beta=f \uparrow(2 j+1)$. Suppose that $\widehat{\varphi}_{j}$ is the $\Delta_{2}^{0}$ characteristic function of $\left\{i: W_{i} \cap \bar{D}\right\}$. Hence, for all $i, \lim _{s} \widetilde{\varphi}_{j, s}(i)$ exists and equals $\widehat{\varphi}_{j}(i)$. (Here $\widehat{\varphi}_{j}$ and $\widetilde{\varphi}_{j, s}(i)$ were defined in 3.1 )

Suppose $\widetilde{\varphi}_{j, s}(g(\beta)) \downarrow=0$ for all $s \geq s_{0}$ for some $s_{0}$. Since $\beta \prec f$ there is some stage $s_{1}>s_{0}$ after which $\beta$ is never initialized. Also for all $\alpha, \alpha \widehat{\alpha} 0 \prec \beta, \widehat{\psi}_{\alpha}$ is total by Lemma 3.3. Hence, $\beta$ can wait for a witness $y=x_{n}^{\beta}$ in (72) to appear at some stage $x>s_{1}$. Then according to Case 3 , Subcase $1, \beta$ will enumerate $x_{n}^{\beta}$ into $Z_{\beta}=W_{g(\beta)}$ causing $Z_{\beta} \cap \bar{D} \neq \emptyset$. Since $\widetilde{\varphi}_{j, t}(g(\beta))=\widetilde{\varphi}_{j, s}(g(\beta))$ for all $t>s$, $\beta$ will never enumerate $x_{n}^{\beta}$ into $D$, so $\lim _{s} \widetilde{\varphi}_{j, s}(g(\beta))=0$, but $W_{g(\beta)} \cap \bar{D} \neq \emptyset$, a contradiction.

Next suppose $\lim _{s} \widetilde{\varphi}_{j, s}(g(\beta)) \downarrow=1$ for all $s \geq s_{0}$ for some $s_{0}$. For every $s$ there can be at most one element in $Z_{s}^{\beta}-D_{s}$ and that must be $\Gamma_{s}=x_{n}^{\beta}$ for some $n$. Since $\beta \prec f$ we know that every $\xi$ with $\xi^{\prec} 0 \prec \beta$ will have the $\Pi_{2}$-outcome. Hence, for each such $x_{n}^{\beta}$, there will come a stage by (73) at which $x_{n}^{\beta}$ is enumerated in $D$ under Subcase 2. But no new $\beta$-witness can be appointed after stage $s_{0}$ under Subcase 1. Hence, $\lim _{s} \widetilde{\varphi}_{j, s}(g(\beta)) \downarrow=1$, but $W_{g(\beta)} \cap \bar{D}=\emptyset$, a contradiction.

While we do not use this theorem itself in our proof of Theorem 1.3, we will have the same positive requirements in our theorem, as well as a nearly identical interaction between the positive and negative requirements. In the proof of Theorem 1.3. notice the similarity between Lemma 3.3 above and Lemma 8.8 . More directly, Lemma 3.4 appears below with some minor changes as Lemma 8.16

\section{Proof Strategy}

As in Theorem 3.1, we will build our c.e. set $D$ on a tree. The positive requirements that make $D$ nonlow are exactly the same as in the theorem above. However, the negative requirements will now be part of the automorphism construction. Our tree will be much more intricate than the tree used in Theorem 3.1 because it will include the entire automorphism construction. 
Recall that we are trying to show the following theorem:

Theorem 1.3. There is a nonlow c.e. set D such that for all c.e. $A \leq_{\mathrm{T}} D$, there exists a low c.e. set $B$ and an automorphism of $\mathcal{E}$ taking $A$ to $B$.

We will consider the case for a single set $A=\Psi^{D}=W_{i}$. The theorem follows as explained in 4.7

4.1. The negative requirements. Let us first examine the special case where $D=A$. The set $B$ is low, which means that it satisfies a stability property saying that if infinitely many elements enter $W_{e}$ before entering $B$, then infinitely many elements remain in $W_{e} \cap \bar{B}$. In order to guarantee an automorphism taking $A$ to $B$, we will need to match the flow of elements into $A$ with a flow of elements into $B$, so we will need some sort of stability on $A$ to meet the stability of $B$. However, if we guarantee this stability property on the whole tree, we would in fact have that $\bar{A}$ was semi-low, which is exactly what the positive requirements are guaranteeing that it is not. What we will actually achieve is stability along the true path only. Because the stability is only along the true path, $A$ will not be semi-low, but will have enough stability to build the automorphism. In Theorem 3.1 this is analogous to the pseudo-requirement of making $D$ low in order to actually achieve that $D$ is just low 2 .

For the general case when $A \neq D$, the pseudo-requirement of stability will be the same. However, we cannot restrain elements from $A$ directly, so we will instead restrain $D$, which will in turn keep the desired elements out of $A$, via the reduction $A=\Psi^{D}$.

4.2. The Harrington-Soare automorphism method. We must build a nonlow set $D$, a low set $B$, and an automorphism from $A=\Psi^{D}$ to $B$. We modify the Harrington-Soare automorphism method as explained below, while simultaneously adding elements to $D$ to ensure that $D$ is nonlow and restraining $D$ to ensure that $A$ is automorphic to $B$.

Harrington and Soare developed their $\Delta_{3}^{0}$ automorphism construction in [4. The construction is performed on a computable tree. Take the lattice $\mathcal{E}$ and quotient out by the ideal of finite sets to get the lattice $\mathcal{E}^{*}$ of c.e. sets up to finite difference. Soare [15, page 343] showed that if there is an automorphism of $\mathcal{E}^{*}$ taking $A$ to $B$, then there is one of $\mathcal{E}$ as well, so we can work in $\mathcal{E}^{*}$. We first make two lists $\left\{U_{i}\right\}_{i \in \omega}$ and $\left\{V_{i}\right\}_{i \in \omega}$ of all the c.e. sets up to finite difference. We let $U_{0}=A$. We imagine the $U_{i}$ sets as being on the $A$-side of the construction, and the $V_{i}$ sets as being on the $B$-side. We build c.e. sets $\left\{\widehat{U}_{i}\right\}_{i \in \omega}$ and $\left\{\widehat{V}_{i}\right\}_{i \in \omega}$ such that there is an automorphism taking $U_{i}$ to $\widehat{U_{i}}$. The inverse of the automorphism will take $V_{i}$ to $\widehat{V}_{i}$. We define $B$ to be $\widehat{U_{0}}$.

The e-state of an element $x$ tells us which c.e. sets $U_{i}$ and $\widehat{V}_{i}$, or $\widehat{U_{i}}$ and $V_{i}$, contain the element $x$, for all $i<e$. In order to build an automorphism taking a set $A$ to a set $B$, it suffices to ensure that the same $e$-states contain infinitely many elements on each side of the construction.

We build two identical trees, $T$ and $\widehat{T}$, according to rules that will be explained later. Elements move through the nodes on the trees, starting from the root. If $x$ is at node $\alpha$ or some node $\delta$ that extends $\alpha$ on $T$, then $x$ can be enumerated into the sets $U_{\alpha}$ and $\widehat{V_{\alpha}}$, where $U_{\alpha}={ }^{*} U_{e_{\alpha}}$ for some $e_{\alpha} \in \omega$ such that the coding is invariant 
over nodes of the same length and similarly for elements on $\widehat{T}$. The automorphism we build takes $U_{\alpha}$ to $\widehat{U_{\alpha}}$ and $V_{\alpha}$ to $\widehat{V_{\alpha}}$ for all $\alpha$ on the true path, which is $\Delta_{3}^{0}$.

We can think of the construction as a game between the Red and Blue players. The Red player (thought of as the opponent) controls the sets $U_{i}$ and $V_{i}$, and the Blue player (thought of as us) controls $\widehat{U}_{i}$ and $\widehat{V}_{i}$. The Blue player wins if the sets define an automorphism. In the Harrington-Soare construction of 4], if Red enumerates an element $x$ at node $\alpha$ into the set $A=U_{0}$, then Blue matches it by enumerating the first available $\widehat{x}$ into $B$ such that $x$ and $\widehat{x}$ are in the same $e_{\alpha}$-state.

However, in our theorem, we want $B$ to be low, so Blue cannot be allowed to choose any element to put into $B$. It is problematic if Red enumerates so many elements into $A$ that Blue must either ignore the lowness restraint on $B$ or fail to put enough elements into $B$ to build an automorphism. We must therefore ensure that Red always leaves infinitely many elements from every $e$-state in $\bar{A}$. In other words, we must satisfy the stability property from 4.1 at least along the true path.

4.3. The modified automorphism method. We use a method similar to the Harrington-Soare $\Delta_{3}^{0}$ automorphism construction. However, we must make $B$ low, which means that we must have a restraint on $B$ to guarantee stability. In the Harrington-Soare construction of [4, elements moved among the nodes on two copies of our tree of strategies, corresponding to the $A / \bar{A}$ and $B / \bar{B}$ sides of the construction. Now, if we let $A$ have free rein, elements may enter $A$ that will not be matched on the $B$ side due to the restraint on $B$. We must therefore keep elements in $\bar{A}$ by somehow restraining $A$. As we don't control $A$ itself, the only way to do this is to put a restraint on the set $D$.

In our new construction, we build four identical trees, corresponding to $\bar{A}, A, \bar{B}$, and $B$. Elements move through the $\bar{A}$ and $\bar{B}$ trees until they are enumerated into $A$ or $B$, when they switch to the corresponding tree. We now must take into account the fact that elements are disappearing from two of the trees and appearing in the others. These mechanics have not appeared before now.

In addition to the nodes on the tree that deal with the automorphism, we must put nodes on the tree corresponding to the positive requirement of making $D$ nonlow. The automorphism nodes themselves act as negative requirement nodes. They tell us which elements we want to restrain to keep out of $A$.

As mentioned previously, the mechanics of the positive and negative requirements are very similar to those involved in constructing a nonlow c.e. set that is $\operatorname{low}_{2}$ as in $\$ 3$.

4.4. A tree argument for automorphisms. We perform our construction on a tree as in $\$ 3.5$, but with the addition of automorphism nodes that will also act as negative requirement nodes. We shall define in $\$ 6.2$ a computable tree $T$ with true path $f$. If $\alpha \in T$ and $\alpha \widehat{\alpha} a \prec f$, then $a$ is the correct guess at the outcome of $\alpha$.

For each $n \in \omega$ there is some $m_{n} \in \omega$ such that for every $\alpha \in T$ of length $m_{n}, \widehat{U}_{\alpha}$ will be a potential candidate for $\widehat{U}_{n}$ and if $\alpha \prec f$, then $U_{\alpha}={ }^{*} U_{n}$ and $\widehat{U}_{\alpha}$ will be the correct candidate for $\widehat{U}_{n}$. Thus, $f$ will specify the sequence $\left\{\widehat{U}_{f} \mid m_{n}\right\}_{n \in \omega}$ which will be the desired sequence $\left\{\widehat{U}_{n}\right\}_{n \in \omega}$ and similarly for $V_{n}$. In a tree construction $f$ is not in general computable but only $\mathbf{0}^{\prime \prime}$-computable, so the sequences $\left\{\widehat{U}_{n}\right\}_{n \in \omega}$ and $\left\{\widehat{V}_{n}\right\}_{n \in \omega}$ will only have a $\mathbf{0}^{\prime \prime}$-computable (i.e., $\Delta_{3}^{0}$ ) presentation.

We say an $e$-state is well-visited if infinitely many elements enter that state during the construction. An $e$-state is well-resided if infinitely many elements remain in 
that state, and emptied if it is well-visited but not well-resided. If $\alpha \in T$ and $|\alpha|=5 e+j$, for $0<j \leq 4$, then associate with $\alpha$ a version of the automorphism strategy. Each $\alpha$ will have associated sets $\mathcal{M}_{\alpha}, \mathcal{B}_{\alpha}$, and $\mathcal{R}_{\alpha}$, where $\mathcal{M}_{\alpha}$ is a set of $e$-states, $\mathcal{R}_{\alpha} \subseteq \mathcal{M}_{\alpha}$, and $\mathcal{B}_{\alpha} \subseteq \mathcal{M}_{\alpha}-\mathcal{R}_{\alpha}$. These represent $\alpha$ 's guess at which states are well-visited, states whose emptying is attributed to the Red player, and states whose emptying is attributed to the Blue player, respectively. In addition each $\alpha$ has an associated $k_{\alpha} \in \omega$, which represents $\alpha$ 's guess at the least element such that no larger element will ever enter a non-well-visited $e$-state. Further restrictions for which nodes appear on the tree are given in Definition 6.2.

Each of the sets $\mathcal{M}_{\alpha}$, etc., will be divided into two subsets $\mathcal{M}_{\alpha}^{A}$ and $\mathcal{M}_{\alpha}^{\bar{A}}$, depending on whether or not $A=U_{0}$ is in each state. We can think of these as being on two different trees, for $A$ and $\bar{A}$. In addition, we will have duals $\widehat{\mathcal{M}}_{\alpha}$, etc., corresponding to $B$ and $\bar{B}$. As mentioned in 4.3 , we will refer to these as four separate, but identical, trees, one corresponding to each of $A, \bar{A}, B$, and $\bar{B}$.

If $\beta \in T$ and $|\beta|=5 e+4$, then associate with $\beta$ a version of the strategy for $P_{e}$, and put the outcomes $\beta^{\wedge} 0$ and $\beta^{\wedge} p$ for all $p \geq 1$ on $T$, representing the $\Pi_{2}$ outcome and all possible $\Sigma_{2}$-outcomes of the $\beta$-strategy. The nodes below $\beta$ have order type $1+\omega^{*}$ with $\beta^{\wedge} 0$ on the left, and the others to the right arranged in the order of $\omega^{*}$. Note that the nodes guessing at the positive strategy are of length $5 e+5$, so these do not conflict with $\beta$ 's own guesses for the automorphism requirements. We also associate $\beta^{\frown} p$ with $\mathcal{M}_{\beta^{\wedge} p}=\mathcal{M}_{\beta}$, etc. The true path $f$ on $T$ and its computable approximation $\left\{f_{s}\right\}_{s \in \omega}$ such that $f=\liminf _{s} f_{s}$ will be defined below.

Suppose $\beta \in T$ and $|\beta|=5 j+4$. Then $\beta$ has an associated witness function $\Gamma_{s}^{\beta}$ for carrying out its strategy for $P_{j}$ as in the construction of a low ${ }_{2}$ set that is not low in $\$ 3.2$

Suppose $\alpha \in T$. Let $A=\Psi^{D}$. The stage $s$ guess at whether $y$ is in $A$ is $\Psi^{D}(y)[s]=\Psi_{s}^{D_{s}}(y)$. The use of this computation is denoted by $\psi_{s}(y)$. The following functions are modifications of those in (7) and (8). Define

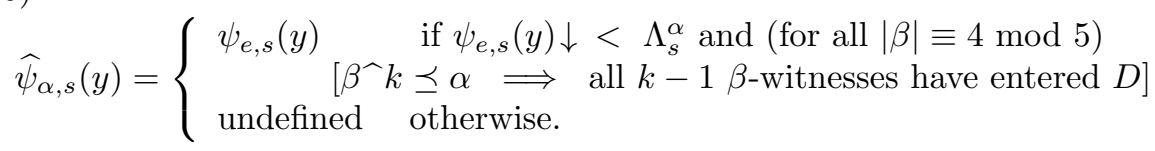

For notational simplicity, we will write $\widehat{\Psi}_{\alpha}(x)[s]=0$ to denote $\Psi^{D}(x)[s]=0$ and $\widehat{\psi}_{\alpha, s}(x) \downarrow$. This means that at stage $s, D_{s}$ believes that $x \notin A$, and all nodes $\beta \prec \alpha$ will no longer injure the computation.

4.5. Making $\mathcal{L}^{*}(A) \cong \mathcal{L}^{*}(B)$. We know by Soare [14 that since $B$ is low, $\mathcal{L}^{*}(B) \cong$ $\mathcal{E}^{*}$, where $\mathcal{L}^{*}(C)$ is the lattice of c.e. sets modulo finite difference intersected with the complement of $C$ (this is isomorphic to the lattice of c.e. sets modulo finite difference containing $C$ ). The negative requirements stem from the automorphism requirements, which must guarantee that $\mathcal{L}^{*}(A) \cong \mathcal{L}^{*}(B)$. We use a modified version of the automorphism construction in Harrington and Soare [4]. Elements flow through the tree as in that paper, but when they enter $A=U_{0}$, they disappear from the tree (as they switch from the $\bar{A}$-tree to the $A$-tree).

We would like to match the well-resided $e$-states that do not contain $A$ with the well-resided $e$-states that do not contain $B$. However, elements may be falling into 
$A$ that we cannot match by putting elements into $B$ because $B$ is low. Thus, we need to restrain elements in $\bar{A}$ so that no well-visited state in $\mathcal{M}_{\alpha}^{\bar{A}}$ is emptied by $A$ alone. In other words, we need stability.

For each $k \in \omega$ and $\nu_{e} \in \mathcal{M}_{\alpha}$, we will choose an element $y_{\langle e, k\rangle} \in \nu_{e}$ and restrain $D$ to keep $y_{\langle e, k\rangle}$ out of $A$. This restraint will be the modified use function $\widehat{\psi}_{\alpha}\left(y_{\langle e, k\rangle}\right)[s]$. In fact, we will not put $x$ into any set $\widehat{V}_{\alpha}$ or $U_{\alpha}$ unless $\widehat{\Psi}_{\alpha}^{D}(x)[s]=0$.

For a given $e$ with $\nu_{e} \in \mathcal{M}_{\alpha}^{\bar{A}}$ and stage $s$, define $y_{\langle e, k\rangle}^{s}$ by recursion on $k$. This is our current guess at $y_{\langle e, k\rangle}$, where $y_{\langle e, k\rangle}$ is an element permanently in $\bar{A}$. Suppose $y_{\langle e, i\rangle}^{s}$ has been defined for all $i<k$. Let $y_{\langle e, k\rangle}^{s}=y_{\langle e, k\rangle}^{s-1}$ if $\widehat{\Psi}_{\alpha}\left(y_{\langle e, k\rangle}^{s-1}\right)[s-1]=0$. Otherwise, check if there is a $y$ that entered $\nu_{e}$ at stage $s$ under Step 1 of the construction in $\$ 7.3$ (or Step 6C if $\alpha=\lambda$ ) such that

$$
y>\max \left\{y_{\langle e, k-1\rangle}^{s}, y_{\langle e, k\rangle}^{t} \mid t<s\right\}, y \in \nu_{e, s}, \text { and } \widehat{\Psi}_{\alpha}(y)[s-1]=0 .
$$

If such a $y$ exists, set it as $y_{\langle e, k\rangle}^{s}$. Otherwise, $y_{\langle e, k\rangle}^{s}$ is undefined. To summarize, $y_{\langle e, k\rangle}^{s}$ is a value that enters $\nu_{e}$ under Step 1 and appears to not be in $A$. It stays as $y_{\langle e, k\rangle}^{s}$ until it no longer appears to be in $\bar{A}$.

For a positive requirement $\beta$ of lower priority, $\beta$ can appoint the $n^{\text {th }}$ witness, $x_{n}^{\beta}$, only after $\widehat{\psi}_{\alpha, s}\left(y_{\langle e, k\rangle}\right) \downarrow$ for all $\langle\alpha, e, k\rangle \leq\langle\beta, n\rangle$, and must exceed their values. (Here, we identify the nodes of $T$ with their Gödel numbering under some fixed effective numbering of $T$.) We will show that for each $e$ and $k$ the computation $\widehat{\psi}_{\alpha, s}\left(y_{\langle e, k\rangle}^{s}\right)$ can be injured at most finitely often. The proof is similar to the proof that the negative requirements are satisfied in the construction of a $\operatorname{low}_{2}$ set that is nonlow.

4.6. Building $B$. We must also build $B$ low such that $\mathcal{M}_{\alpha}^{A}=\widehat{\mathcal{M}}_{\alpha}^{B}$. That is, we want to match the well-visited states that contain $A$ with the well-visited states that contain $B$. So far we have only discussed keeping elements out of $A$, but not putting elements into $B$. We will ensure $\mathcal{G}_{\alpha}=\widehat{\mathcal{G}}_{\alpha}$, where

$$
\mathcal{G}_{\alpha}=\left\{\nu \mid\left(\exists^{\infty} x\right)(\exists s)\left[x \in A_{\text {at } s} \text { and } \nu(\alpha, x, s)=\nu\right]\right\}
$$

and $\widehat{\mathcal{G}}_{\alpha}$ is defined similarly for $B$ in place of $A$. We can then force $\mathcal{M}_{\alpha}^{A}=\widehat{\mathcal{M}}_{\alpha}^{B}$ by performing the automorphism steps on elements that have already entered $A$ or $B$.

To make $B$ low, we will meet the negative requirement:

$$
N_{j}^{B}: \quad\left(\exists^{\infty} s\right) \Phi_{j}^{B}(j)[s] \downarrow \Longrightarrow \Phi_{j}^{B}(j) \downarrow .
$$

In addition, we must ensure that no well-visited state is emptied by $B$. To satisfy this, we will wait for two new elements to come into the state before allowing one of them to enter $B$.

For the positive requirement, we need to ensure that $\mathcal{G}_{\alpha}=\widehat{\mathcal{G}}_{\alpha}$. To do this, we meet:

$$
P_{e, x}^{B}: \quad x \text { enters } A \text { from state } \nu_{e} \Longrightarrow(\exists y)\left[y \text { enters } B \text { from } \widehat{\nu}_{e}\right]
$$

When $x$ enters $A$ from $\alpha$-state $\nu_{e}$, we add $\langle\alpha, x, e\rangle$ to a list $\Lambda$, and later find a witness $y$ to enumerate into $B$ in the state $\widehat{\nu}_{e}$. 
4.7. The hidden master tree. In order to show that $\overline{\mathbf{L}}_{1}$ is noninvariant, we must show that for every c.e. $A \leq_{\mathrm{T}} D$, there is a low $B$ such that $A$ is automorphic to $B$. However, so far we have only discussed this for a single $A=W_{i}=\Psi^{D}=\Phi_{e}^{D}$. To show this for all $A \leq_{\mathrm{T}} D$, we will use a much bigger master tree with infinitely many levels on the tree corresponding to each pair $\langle e, i\rangle$, representing the possibility that $A=W_{i}=\Phi_{e}^{D}$.

Let $n_{\langle e, i\rangle}$ be the least of any of the lengths corresponding to $\langle e, i\rangle$. Then nodes of length $n_{\langle e, i\rangle}$ will be extended by guesses at whether we believe $\Phi_{e}^{D}=W_{i}$. Let $\alpha$ have length $n_{\langle e, i\rangle}$. Then $\widehat{\alpha} 0$ represents the guess that $W_{i}=\Phi_{e}^{D}$, and $\widehat{\alpha^{\wedge} 1 \text { represents }}$ the guess that $W_{i} \neq \Phi_{e}^{D}$. We say $s$ is an $\alpha$-state if the approximation to the true path at stage $s$ contains $\alpha$. If $s$ is an $\alpha$-state and the length of agreement between $W_{i, s}$ and $\Phi_{e}^{D}[s]$ has increased since the previous $\alpha$-state, we let our approximation to the true path at stage $s$ go through $\alpha^{\wedge} 0$, and otherwise, through $\alpha^{\wedge} 1$. For each $\alpha$ of length $n_{\langle e, i\rangle}$, we create a tree $T_{\alpha}$ made up of the nodes affiliated with $\langle e, i\rangle$ that extend $\alpha^{\curlyvee} 0$ as well as all positive requirement nodes extending $\alpha^{\wedge} 0$. Note that $T_{\alpha}$ will already be guessing at all positive requirement nodes that $\alpha$ extends. If these guesses are incorrect, then only finitely much activity will occur on $T_{\alpha}$. The automorphism machinery for $\langle e, i\rangle$ will only act at stages when the approximation to the true path goes through $\alpha \widehat{\Upsilon} 0$. This machinery acts upon the tree $T_{\alpha}$. This is the tree mentioned in 4.4 and throughout the rest of the proof, for a fixed $e, i$, and $\alpha$.

The ordering of the nodes on the master tree is as follows: height 1 nodes guess at whether $W_{0}=\Phi_{0}^{D}$, and the next four levels correspond to the first four automorphism requirement nodes on the tree $T_{0}=T_{\langle 0,0\rangle}$, followed by height 6 nodes, which are guesses at the number of witnesses for $P_{0}$. These are followed by the guess for $\langle e, i\rangle=1$, then the second four automorphism levels of $T_{0}$ and the first four automorphism levels of $T_{1}$, and then $P_{1}$. Thus, the space between the $P_{i}$ 's increases as more and more automorphism nodes are added.

In the end, the trees $T_{\alpha}$ will look and act exactly as the tree $T$ in the other sections of this proof with one small difference. Each positive node will need to respect finitely many restraints put on by the finitely many additional $T_{\alpha^{\prime}}$, for $\alpha^{\prime}<_{L} \alpha$. As the restraint is finite, this is a negligible change.

To summarize, the addition of multiple $A$ 's does not change the theorem except that it adds more nodes to the tree that must obey the same rules. It will cause $D$ to be restrained more. The case for a single $e$ and $i$ is the heart of the problem. We need not refer back to this master tree, as it simply tells us that we may perform the construction for a specific $A=W_{i}=\Phi_{e}^{D}$ and that we may weave these constructions together to get the general proof.

\section{AN OVERVIEW OF THE AUTOMORPHISM MACHINERY}

This section, which contains important definitions, is largely taken from the Harrington-Soare $\Delta_{3}^{0}$ automorphism paper [4. The few changes are primarily in differentiating between the $A$ and $\bar{A}$ parts of the proof.

5.1. Background. As mentioned previously, by [15, page 343], building an automorphism of $\mathcal{E}$ is equivalent to building one of $\mathcal{E}^{*}$, the quotient lattice of $\mathcal{E}$ modulo the ideal $\mathcal{F}$ of finite sets. To do this we fix two copies of the natural numbers $\omega$ and $\widehat{\omega}$. We let variables $x, y, \ldots(\hat{x}, \hat{y}, \ldots)$ range over $\omega(\widehat{\omega})$. Occasionally, we shall 
specify the definitions and action for only the $\omega$-side, meaning that the $\widehat{\omega}$-side will be entirely dual or simplified in an obvious way. However, there are quite a few differences between the $\omega$ and $\widehat{\omega}$ sides that were not needed in the Harrington-Soare paper [4], and we will make these differences explicit.

As mentioned in $\$ 4.2$, we view the construction of the automorphism $\Phi$ as a game between two players in the sense of Lachlan [7. The Red player produces two standard indexings $\left\{U_{n}\right\}_{n \in \omega}$ and $\left\{V_{n}\right\}_{n \in \omega}$ of the c.e. sets (up to finite difference), where we view $U_{n}$ as being on the $\omega$-side and $V_{n}$ on the $\widehat{\omega}$-side. The Blue player responds by building c.e. sets $\left\{\widehat{U}_{n}\right\}_{n \in \omega}$ on the $\widehat{\omega}$-side and $\left\{\widehat{V}_{n}\right\}_{n \in \omega}$ on the $\omega$-side. The condition necessary to show that this correspondence $\Phi\left(U_{n}\right)=\widehat{U}_{n}$ and $\widehat{V}_{n}=\Phi^{-1}\left(V_{n}\right)$ is an automorphism is best stated in terms of the following notion of full $e$-state. We restrict our indexings $\left\{U_{n}\right\}_{n \in \omega}$ to those where $U_{0}=W_{i}=A$.

Definition 5.1. Given two sequences of c.e. sets $\left\{X_{n}\right\}_{n \in \omega}$ and $\left\{Y_{n}\right\}_{n \in \omega}$, define $\nu(e, x)$, the full $e$-state of $x$ with respect to (w.r.t.) $\left\{X_{n}\right\}_{n \in \omega}$ and $\left\{Y_{n}\right\}_{n \in \omega}$ to be the triple $\langle e, \sigma(e, x), \tau(e, x)\rangle$, where

$$
\begin{gathered}
\sigma(e, x)=\left\{i: i \leq e \& x \in X_{i}\right\}, \text { and } \\
\tau(e, x)=\left\{i: i \leq e \& x \in Y_{i}\right\} .
\end{gathered}
$$

To see that $\Phi$ is an automorphism it suffices to satisfy the requirement,

$$
\begin{aligned}
& (\forall \nu)\left(\exists^{\infty} x \in \omega\right)\left[\nu(e, x)=\nu \text { w.r.t. }\left\{U_{n}\right\}_{n \in \omega} \text { and }\left\{\widehat{V}_{n}\right\}_{n \in \omega}\right] \\
& \Longleftrightarrow\left(\exists^{\infty} \hat{y} \in \hat{w}\right)\left[\nu(e, \hat{y})=\nu \text { w.r.t. }\left\{\widehat{U}_{n}\right\}_{n \in \omega} \text { and }\left\{V_{n}\right\}_{n \in \omega}\right] .
\end{aligned}
$$

Definition 5.2. Given computable enumerations $\left\{X_{s}\right\}_{s \in \omega}$ and $\left\{Y_{s}\right\}_{s \in \omega}$ of c.e. sets $X$ and $Y$, define $X \backslash Y=\left\{z:(\exists s)\left[z \in X_{s}-Y_{s}\right]\right\}$.

5.2. The $\alpha$-section $S_{\alpha}, \alpha$-region $R_{\alpha}$, and c.e. set $Y_{\alpha}$. We divide up the $\omega$-side into disjoint $\alpha$-sections, $S_{\alpha}$, for $\alpha \in T$. We shall define during the construction in $\$ 7$ a function $\alpha(x, s)$ with range $T$ which indicates that $x$ is in section $S_{\alpha(x, s)}$ at the end of stage $s$, and we shall guarantee that $\alpha(x)=\lim _{s} \alpha(s, x)$ exists. The $\alpha$-region $R_{\alpha}$ consists of all $S_{\gamma}$ such that $\alpha \preceq \gamma$. For each stage $s$ we define

$$
\begin{gathered}
S_{\alpha, s}=\{x: \alpha(x, s)=\alpha\}, \\
R_{\alpha, s}=\{x: \alpha \preceq \alpha(x, s)\}, \text { and } \\
Y_{\alpha, s}=\bigcup\left\{R_{\alpha, t}: t \leq s\right\} .
\end{gathered}
$$

Define $S_{\alpha, \infty}=\{x: \alpha(x)=\alpha\}$ and $R_{\alpha, \infty}=\{x: \alpha(x) \preceq \alpha\}$. Note that these sets contain all $x$ in either the $A$-tree or the $\bar{A}$-tree. When we need to distinguish between them, we will write them as $S_{\alpha, s}^{A}, S_{\alpha, s}^{\bar{A}}$, etc.

We shall guarantee that for all $\alpha \in T, \alpha \neq \lambda$,

$$
\begin{gathered}
Y_{\alpha} \backslash Y_{\alpha^{-}}=\emptyset \text {, and } \\
\alpha \prec f \Longrightarrow \begin{array}{c}
R_{\alpha, \infty}={ }^{*} Y_{\alpha}={ }^{*} \omega, \\
R_{\alpha, \infty}^{A}={ }^{*} Y_{\alpha}^{A}={ }^{*} A, \text { and } \\
R_{\alpha, \infty}^{\bar{A}}={ }^{*} Y_{\alpha}^{\bar{A}}={ }^{*} \bar{A} .
\end{array}
\end{gathered}
$$

We shall ensure (26) by making $x$ enter $S_{\alpha^{-}}$before $x$ enters $R_{\alpha}$. Also $x$ will enter $R_{\alpha}$ at most once (although $x$ may later leave $R_{\alpha}$ ). During the construction in $\$ 7$ we shall define a computable sequence $\left\{f_{s}\right\}_{s \in \omega}$ such that $f=\liminf _{s} f_{s}$. 
If $f_{s}<_{L} \alpha$ for some $s \geq x$ we say $x$ is $\alpha$-ineligible at all stages $t \geq s$, and we insist that $x \notin S_{\alpha, t}$. Hence, $R_{\alpha, \infty}=\emptyset$ for all $\alpha$ with $f<_{L} \alpha$. Secondly, $Y_{\alpha}$ will be finite for all $\alpha<_{L} f$. Finally, $S_{\alpha, \infty}$ will be finite for all $\alpha$. These three facts imply (27).

5.3. The $\alpha$-states $\nu(\alpha, x, s)$, and lists $\mathcal{E}_{\alpha}, \mathcal{F}_{\alpha}, \mathcal{M}_{\alpha}$. For conceptual simplicity we do as little action as possible at each node $\alpha \in T$. If $|\alpha| \equiv 1 \bmod 5(|\alpha| \equiv 2 \bmod 5)$, we consider one new $U$ set $(V$ set $)$. If $|\alpha| \equiv 3 \bmod 5(|\alpha| \equiv 4 \bmod 5)$, we consider new $\alpha$-states $\nu(\hat{\nu})$ which may be non-well-resided on $Y_{\alpha}\left(\widehat{Y}_{\alpha}\right)$. If $|\alpha| \equiv 0 \bmod 5$ we make no new commitments for the automorphism machinery but we instead act for the positive requirements. We shall arrange for all $n \in \omega$ that for $\alpha \prec f$,

$$
\begin{gathered}
|\alpha|=5 n+1 \Longrightarrow U_{\alpha}={ }^{*} U_{n}, \text { and } \\
|\alpha|=5 n+2 \Longrightarrow V_{\alpha}={ }^{*} V_{n} .
\end{gathered}
$$

We let $U_{\alpha}$ and $\widehat{U}_{\alpha}\left(V_{\alpha}\right.$ and $\left.\widehat{V}_{\alpha}\right)$ be undefined if $|\alpha| \not \equiv 1 \bmod 5(|\alpha| \not \equiv 2 \bmod 5)$. We let $e_{\alpha}\left(\hat{e}_{\alpha}\right)$ correspond to $n$ in (28) (respectively (29)). Specifically, define $e_{\lambda}=$ $\hat{e}_{\lambda}=-1$ and if $|\alpha| \equiv 1 \bmod 5$, then let $e_{\alpha}=e_{\alpha^{-}}+1$, and otherwise let $e_{\alpha}=e_{\alpha^{-}}$. Define $\hat{e}_{\alpha}$ similarly with $|\alpha| \equiv 2 \bmod 5$ in place of $|\alpha| \equiv 1 \bmod 5$. Hence, $e_{\alpha}>e_{\alpha^{-}}$ $\left(\hat{e}_{\alpha}>\hat{e}_{\alpha^{-}}\right)$if and only if $|\alpha| \equiv 1 \bmod 5(|\alpha| \equiv 2 \bmod 5)$.

Definition 5.3. An $\alpha$-state is a triple $\langle\alpha, \sigma, \tau\rangle$ where $\sigma \subseteq\left\{0, \ldots, e_{\alpha}\right\}$ and $\tau \subseteq$ $\left\{0, \ldots, \hat{e}_{\alpha}\right\}$. The only $\lambda$-state is $\nu_{-1}=\langle\lambda, \emptyset, \emptyset\rangle$.

The construction in $\$ 7$ will produce a simultaneous computable enumeration $U_{\alpha, s}, V_{\alpha, s}, \widehat{U}_{\alpha, s}, \widehat{V}_{\alpha, s}$, for $\alpha \in T$ and $s \in \omega$, of these c.e. sets which we use in the following definition.

Definition 5.4. (i) The $\alpha$-state of $x$ at stage $s, \nu(\alpha, x, s)$, is the triple $\langle\alpha, \sigma(\alpha, x, s)$, $\tau(\alpha, x, s)\rangle$, where

$$
\begin{aligned}
& \sigma(\alpha, x, s)=\left\{e_{\beta}: \beta \preceq \alpha \& e_{\beta}>e_{\beta^{-}} \& x \in U_{\beta, s}\right\}, \\
& \tau(\alpha, x, s)=\left\{\widehat{e}_{\beta}: \beta \preceq \alpha \& \widehat{e}_{\beta}>\widehat{e}_{\beta^{-}} \& x \in \widehat{V}_{\beta, s}\right\} .
\end{aligned}
$$

(ii) The final $\alpha$-state of $x$ is $\nu(\alpha, x)=\langle\alpha, \sigma(\alpha, x), \tau(\alpha, x)\rangle$, where $\sigma(\alpha, x)=$ $\lim _{s} \sigma(\alpha, x, s)$ and $\tau(\alpha, x)=\lim _{s} \tau(\alpha, x, s)$.

For each $\alpha \in T$ we define the following sets of $\alpha$-states called lists:

$$
\mathcal{E}_{\alpha}^{C}=\left\{\nu:\left(\exists^{\infty} x\right)(\exists s)\left[x \in S_{\alpha, s}^{C}-\bigcup\left\{S_{\alpha, t}: t<s\right\} \quad \& \nu(\alpha, x, s)=\nu\right]\right\},
$$

where $C$ is either $A$ or $\bar{A}$. We define $\mathcal{E}_{\alpha}=\mathcal{E}_{\alpha}^{A} \cup \mathcal{E}_{\alpha}^{\bar{A}}$. Let

$$
\mathcal{F}_{\alpha}=\left\{\nu:\left(\exists^{\infty} x\right)(\exists s)\left[x \in R_{\alpha, s} \& \nu(\alpha, x, s)=\nu\right]\right\} .
$$

We may also define $\mathcal{F}_{\alpha}^{A}$ and $\mathcal{F}_{\alpha}^{\bar{A}}$ by replacing $R_{\alpha, s}$ with $R_{\alpha, s}^{A}$ or $R_{\alpha, s}^{\bar{A}}$. Then $\mathcal{F}_{\alpha}=$ $\mathcal{F}_{\alpha}^{A} \cup \mathcal{F}_{\alpha}^{\bar{A}}$.

Note that $\mathcal{E}_{\alpha}$ consists of states well-visited by elements $x$ when they first enter $R_{\alpha}$, and $\mathcal{F}_{\alpha}$ those states well-visited while they remain in $Y_{\alpha}$, so $\mathcal{E}_{\alpha} \subseteq \mathcal{F}_{\alpha}$. Note that $\mathcal{E}_{\alpha}^{A}$ is the set of all states that are well-visited as entry states by elements that have never been in $S_{\alpha}$. This excludes entry states that are well-visited only by elements switching from $S_{\alpha}^{\bar{A}}$ to $S_{\alpha}^{A}$. However, we will show that in fact these entry states are included, as they will also be well-visited by elements that have not yet been in $S_{\alpha}$. Each $\alpha \in T$ will have an associated list $\mathcal{M}_{\alpha}$ which is roughly 
$\alpha$ 's "guess" at the true $\mathcal{F}_{\alpha}$ such that if $\alpha \prec f$, then $\mathcal{M}_{\alpha}=\mathcal{F}_{\alpha}$. For $\alpha \prec f$ we shall achieve $\mathcal{M}_{\alpha}=\mathcal{F}_{\alpha}$ by ensuring the following properties of $\mathcal{M}_{\alpha}$ :

$$
\mathcal{E}_{\alpha} \subseteq \mathcal{M}_{\alpha}
$$

$$
\begin{aligned}
& \left(\forall^{\infty} x\right)\left[\begin{array}{c}
\text { if } x \in Y_{\alpha, s}, \nu_{0}=\nu(\alpha, x, s) \in \mathcal{M}_{\alpha}, \\
\text { and Red causes enumeration of } x \text { so that } \\
\nu_{1}=\nu(\alpha, x, s+1), \text { then } \nu_{1} \in \mathcal{M}_{\alpha}
\end{array}\right], \\
& \left(\forall^{\infty} x\right)\left[\begin{array}{c}
\text { if } x \in Y_{\alpha, s}, \nu_{0}=\nu(\alpha, x, s) \in \mathcal{M}_{\alpha}, \\
\text { and Blue causes enumeration of } x \text { so that } \\
\nu_{1}=\nu(\alpha, x, s+1), \text { then } \nu_{1} \in \mathcal{M}_{\alpha}
\end{array}\right] .
\end{aligned}
$$

(Here $\left(\forall^{\infty} x\right)$ denotes "for almost every $x$ ".) Blue enumeration which satisfies (32) is called $\alpha$-legal. Two main constraints on Blue's moves will be (30) and (32). Clearly, (30), (31), and (32) guarantee

$$
\mathcal{F}_{\alpha} \subseteq \mathcal{M}_{\alpha}
$$

During Step 1 of the construction in $\$ 7$ we shall promptly pull elements $x \in Y_{\alpha^{-}, s}$ into $S_{\alpha, s+1}$ in order to ensure

$$
\mathcal{M}_{\alpha} \subseteq \mathcal{E}_{\alpha}
$$

Hence, by (33), (34), and $\mathcal{E}_{\alpha} \subseteq \mathcal{F}_{\alpha}$ we have

$$
\mathcal{M}_{\alpha}=\mathcal{F}_{\alpha}=\mathcal{E}_{\alpha} .
$$

On the $\widehat{\omega}$-side we have dual definitions for the above items by replacing $\omega, x, U_{\alpha}$, $\widehat{V}_{\alpha}, A, \bar{A}$ by $\widehat{\omega}, \widehat{x}, \widehat{U}_{\alpha}, V_{\alpha}, B, \bar{B}$, respectively. These dual items will be denoted by $\widehat{\nu}(\alpha, \widehat{x}, s), \widehat{S}_{\alpha}, \widehat{R}_{\alpha}, \widehat{Y}_{\alpha}, \widehat{\mathcal{E}}_{\alpha}, \widehat{\mathcal{F}}_{\alpha}$, and $\widehat{\mathcal{M}}_{\alpha}$. We write hats over the $\alpha$-states, e.g. $\widehat{\nu}_{1}=\nu(\alpha, \widehat{x}, s)$, to indicate $\alpha$-states for elements $\widehat{x} \in \widehat{\omega}$. We shall ensure

$$
\widehat{\mathcal{M}}_{\alpha}=\left\{\widehat{\nu}: \nu \in \mathcal{M}_{\alpha}\right\},
$$

which implies by (35) that the well-visited $\alpha$-states on both sides coincide. We also define $\mathcal{M}_{\alpha}^{A}$, etc. when we restrict to the $A$-tree or the $\bar{A}$-tree.

Definition 5.5. Given $\alpha$-states $\nu_{0}=\left\langle\alpha, \sigma_{0}, \tau_{0}\right\rangle$ and $\nu_{1}=\left\langle\alpha, \sigma_{1}, \tau_{1}\right\rangle$, we have:

(i) $\nu_{0} \leq_{R} \nu_{1}$ if $\sigma_{0} \subseteq \sigma_{1}$ and $\tau_{0}=\tau_{1}$.

(ii) $\nu_{0} \leq_{B} \nu_{1}$ if $\tau_{0} \subseteq \tau_{1}$ and $\sigma_{0}=\sigma_{1}$.

(iii) $\widehat{\nu}_{0} \leq_{R} \widehat{\nu}_{1}$ if $\widehat{\sigma}_{0}=\widehat{\sigma}_{1}$ and $\widehat{\tau}_{0} \subseteq \widehat{\tau}_{1}$.

(iv) $\widehat{\nu}_{0} \leq_{B} \widehat{\nu}_{1}$ if $\widehat{\sigma}_{0} \subseteq \widehat{\sigma}_{1}$ and $\widehat{\tau}_{0}=\widehat{\tau}_{1}$.

(v) $\nu_{0}<_{R} \nu_{1}\left(\nu_{0}<_{B} \nu_{1}\right)$ if $\nu_{0} \leq_{R} \nu_{1}\left(\nu_{0} \leq_{B} \nu_{1}\right)$ and $\nu_{0} \neq \nu_{1}$, and similarly for $\widehat{\nu}_{0}<_{R} \widehat{\nu}_{1}$ and $\widehat{\nu}_{0}<_{B} \widehat{\nu}_{1}$.

The intuition is that if $\nu_{0}=\nu(\alpha, x, s)$ and $\nu_{0}<_{R} \nu_{1}\left(\nu_{0}<_{B} \nu_{1}\right)$, then Red (Blue) can enumerate $x$ in the necessary $U$ sets $(\widehat{V}$ sets $)$ causing $\nu_{1}=\nu(\alpha, x, s+1)$. For $\widehat{\nu}_{0}$ and $\widehat{\nu}_{1}$ the role of $\sigma$ and $\tau$ is reversed because on the $\widehat{\omega}$-side Blue (Red) plays the $\widehat{U}$ sets $(V$ sets $)$, and hence

$$
\left[\nu_{0}<_{R} \nu_{1} \Longleftrightarrow \widehat{\nu}_{0}<_{B} \widehat{\nu}_{1}\right] \&\left[\nu_{0}<_{B} \nu_{1} \Longleftrightarrow \widehat{\nu}_{0}<_{R} \widehat{\nu}_{1}\right]
$$

Definition 5.6. Let $\beta \preceq \alpha \in T, \nu_{0}$ be an $\alpha$-state $\nu_{0}=\left\langle\alpha, \sigma_{0}, \tau_{0}\right\rangle$, and $\mathcal{C}_{\alpha}$ be a set of $\alpha$-states. Define

(i) $\nu_{0} \uparrow \beta=\left\langle\beta, \sigma_{1}, \tau_{1}\right\rangle$, where we define $\sigma_{1}=\sigma_{0} \cap\left\{0, \ldots, e_{\beta}\right\}$ and we define $\tau_{1}=\tau_{0} \cap\left\{0, \ldots, \widehat{e}_{\beta}\right\}$,

(ii) $\nu_{1} \preceq \nu_{0}$ (read " $\nu_{0}$ extends $\nu_{1}$ ") if $\nu_{0} \uparrow \beta=\nu_{1}$, and 
(iii) $\mathcal{C}_{\alpha} \uparrow \beta=\left\{\nu \uparrow \beta: \nu \in \mathcal{C}_{\alpha}\right\}$

(iv) Given a finite set of $\alpha$-states, $\left\{\nu\left(\alpha, \sigma_{i}, \tau_{i}\right)\right\}_{i \in I}$, we then define $\bigcup_{i \in I}\left\{\nu\left(\alpha, \sigma_{i}\right.\right.$, $\left.\left.\tau_{i}\right)\right\}:=\langle\alpha, \sigma, \tau\rangle$, where $\sigma=\bigcup_{i \in I} \sigma_{i}$, and $\tau=\bigcup_{i \in I} \tau_{i}$.

Definition 5.7. A node $\alpha \in T$ is $\mathcal{M}$-inconsistent if $e_{\alpha}>e_{\beta}$, where $\beta=\alpha^{-}$, and there are $\alpha$-states $\nu_{0}<_{B} \nu_{1}$ such that $\nu_{0} \in \mathcal{M}_{\alpha}$ and $\nu_{1} \uparrow \beta \in \mathcal{M}_{\beta}$ but $\nu_{1} \notin \mathcal{M}_{\alpha}$. Otherwise $\alpha$ is $\mathcal{M}$-consistent.

We shall take action in Step 3 of the construction in 97 to ensure that $\alpha$ is $\mathcal{M}$-consistent if $\alpha \prec f$.

5.4. Well-resided $\alpha$-states and the lists $\mathcal{R}_{\alpha}$ and $\mathcal{B}_{\alpha}$. Define the set of wellresided $\alpha$-states,

$$
\mathcal{K}_{\alpha}=\left\{\nu_{1}:\left(\exists^{\infty} x\right)\left[x \in Y_{\alpha} \& \nu(\alpha, x)=\nu_{1}\right]\right\} .
$$

Likewise define $\widehat{\mathcal{K}}_{\alpha}$ for the $\widehat{\omega}$-side. To satisfy the automorphism requirement (25) we must show for $\alpha \prec f$ that

$$
\widehat{\mathcal{K}}_{\alpha}=\left\{\widehat{\nu}: \nu \in \mathcal{K}_{\alpha}\right\} .
$$

To achieve (39) note that unlike $\mathcal{E}_{\alpha}$ and $\mathcal{F}_{\alpha}$ of $\$ 5.3, \mathcal{K}_{\alpha}$ is $\Pi_{3}^{0}$ not $\Pi_{2}^{0}$, so $\alpha$ cannot guess at $\mathcal{K}_{\alpha}$ directly. Instead we guess at disjoint sets $\mathcal{R}_{\alpha}$ and $\mathcal{B}_{\alpha}$ such that for $\alpha \prec f, \mathcal{M}_{\alpha}-\left(\mathcal{R}_{\alpha} \cup \mathcal{B}_{\alpha}\right)=\mathcal{K}_{\alpha}$. These sets, $\mathcal{R}_{\alpha}$ and $\mathcal{B}_{\alpha}$, correspond to those $\nu \in \mathcal{M}_{\alpha}$ that $\alpha$ believes are being emptied by Red and Blue, respectively.

To define $\mathcal{R}_{\alpha}$ and $\mathcal{B}_{\alpha}$ fix $\alpha \in T$, let $\beta=\alpha^{-}$, and assume that $\mathcal{R}_{\gamma}, \mathcal{B}_{\gamma}$ and their duals $\widehat{\mathcal{R}}_{\gamma}, \widehat{\mathcal{B}}_{\gamma}$ have been defined for all $\gamma \prec \alpha$. We decompose $\mathcal{R}_{\alpha}$ into the disjoint union,

$$
\begin{gathered}
\mathcal{R}_{\alpha}=\mathcal{R}_{\alpha}^{\alpha} \sqcup \mathcal{R}_{\alpha}^{<\alpha}, \text { where } \\
\mathcal{R}_{\alpha}^{<\alpha}:=\left\{\nu: \nu \in \mathcal{M}_{\alpha} \& \nu \uparrow \beta \in \mathcal{R}_{\beta}\right\}, \text { and } \\
\mathcal{R}_{\alpha}^{\alpha}:=\mathcal{R}_{\alpha}-\mathcal{R}_{\alpha}^{<\alpha} .
\end{gathered}
$$

Note that $\mathcal{R}_{\alpha}^{<\alpha}$ is determined by $\mathcal{R}_{\beta}, \beta \prec \alpha$, but $\mathcal{R}_{\alpha}^{\alpha}$ may contain new elements and for $\alpha \prec f$ it has the meaning described below in (44). Likewise, let $\mathcal{B}_{\alpha}=\mathcal{B}_{\alpha}^{\alpha} \sqcup \mathcal{B}_{\alpha}^{<\alpha}$, where $\mathcal{B}_{\alpha}^{<\alpha}$ is defined as in (41) but with $\mathcal{B}_{\beta}$ in place of $\mathcal{R}_{\beta}$.

If $|\alpha| \not \equiv 3 \bmod 5$ define $\mathcal{R}_{\alpha}^{\alpha}=\widehat{\mathcal{B}}_{\alpha}^{\alpha}=\emptyset$. If $|\alpha| \equiv 3 \bmod 5$ we let $\mathcal{M}_{\alpha}=\mathcal{M}_{\beta}$ (since $\alpha$-states are $\beta$-states because $e_{\alpha}=e_{\beta}$ and $\left.\widehat{e}_{\alpha}=\widehat{e}_{\beta}\right)$. We define the $\Pi_{2}^{0}$ predicate,

$$
F(\beta, \nu) \equiv(\forall x)\left[\left[x>|\beta| \& x \in Y_{\beta}\right] \Longrightarrow \nu(\alpha, x) \neq \nu\right],
$$

and we allow $\mathcal{R}_{\alpha}^{\alpha} \neq \emptyset$ with the intention that for $\alpha \prec f$,

$$
\mathcal{R}_{\alpha}^{\alpha}=\left\{\nu: \nu \in \mathcal{M}_{\alpha}-\left(\mathcal{R}_{\alpha}^{<\alpha} \cup \mathcal{B}_{\alpha}^{<\alpha}\right) \& F(\beta, \nu)\right\}
$$

We define

$$
\widehat{\mathcal{B}}_{\alpha}^{\alpha}:=\left\{\widehat{\nu}: \nu \in \mathcal{R}_{\alpha}^{\alpha}\right\} .
$$

If $|\alpha| \not \equiv 4 \bmod 5$ define $\widehat{\mathcal{R}}_{\alpha}^{\alpha}=\mathcal{B}_{\alpha}^{\alpha}=\emptyset$. If $|\alpha| \equiv 4 \bmod 5$ we allow $\widehat{\mathcal{R}}_{\alpha}^{\alpha} \neq \emptyset$ (using the duals of (40)-(44) where e.g. in the dual of (43) we use $\widehat{Y}_{\beta}$ in place of $Y_{\beta}$ ), and we define

$$
\mathcal{B}_{\alpha}^{\alpha}:=\left\{\nu: \widehat{\nu} \in \widehat{\mathcal{R}}_{\alpha}^{\alpha}\right\} .
$$

At most one of $\mathcal{R}_{\alpha}^{\alpha}$ and $\widehat{\mathcal{R}}_{\alpha}^{\alpha}$ is nonempty, so by (46), (45), and (44),

$$
\mathcal{R}_{\alpha}^{\alpha} \cap \mathcal{B}_{\alpha}^{\alpha}=\emptyset \&\left(\left(\mathcal{R}_{\alpha}^{\alpha} \cup \mathcal{B}_{\alpha}^{\alpha}\right) \cap\left(\mathcal{R}_{\alpha}^{<\alpha} \cup \mathcal{B}_{\alpha}^{<\alpha}\right)=\emptyset\right),
$$


and hence

$$
\mathcal{R}_{\alpha} \cap \mathcal{B}_{\alpha}=\emptyset .
$$

If $\alpha \prec f$, then $\nu \in \mathcal{R}_{\alpha}$ implies $F\left(\alpha^{-}, \nu\right)$ and hence

$$
\left(\forall \nu \in \mathcal{R}_{\alpha}\right)\left(\forall x \in Y_{\alpha}\right)(\forall s)[\nu(\alpha, x, s)=\nu \Longrightarrow(\exists t>s)[\nu(\alpha, x, t) \neq \nu]] .
$$

It will be Blue's responsibility to change the $\alpha$-state of $x$ if $\nu(\alpha, x, s) \in \mathcal{B}_{\alpha}$ and $x \in R_{\alpha}$. However, $\mathcal{B}_{\alpha} \cap \mathcal{R}_{\alpha}=\emptyset$ so if $\nu(\alpha, x, s)=\nu \in \mathcal{R}_{\alpha}$, then Blue can wait for Red to change the $\alpha$-state of $x$ to meet (49). That is,

$$
\left(\forall \nu \in \mathcal{R}_{\alpha}\right)\left(\forall x \in R_{\alpha}\right)(\forall s)\left[\begin{array}{c}
\text { if } \nu(\alpha, x, s)=\nu, \text { then it is an } \\
\alpha \text {-admissible move for Blue to restrain } \\
x \text { from further Blue enumeration until } \\
(\exists t>s)\left[\nu(\alpha, x, s)<_{R} \nu(\alpha, x, t)\right]
\end{array}\right] .
$$

Definition 5.8. A node $\alpha \in T$ is $\mathcal{R}$-consistent if

$$
\left(\forall \nu_{0} \in \mathcal{R}_{\alpha}\right)\left(\exists \nu_{1}\right)\left[\nu_{0}<_{R} \nu_{1} \quad \& \quad \nu_{1} \in \mathcal{M}_{\alpha}\right]
$$

and $\mathcal{R}$-inconsistent otherwise.

By applying (50), Blue will ensure that $\alpha$ is $\mathcal{R}$-consistent for $\alpha \prec f$. Now (51), (45), and (37) imply for $\alpha \prec f$ that

$$
\left(\forall \hat{\nu}_{0} \in \widehat{\mathcal{B}}_{\alpha}\right)\left(\exists \widehat{\nu}_{1}\right)\left[\widehat{\nu}_{0}<_{B} \widehat{\nu}_{1} \& \widehat{\nu}_{1} \in \widehat{\mathcal{M}}_{\alpha}\right] .
$$

By repeatedly applying (52), Blue can achieve $\widehat{\nu}_{1} \in \widehat{\mathcal{M}}_{\alpha}-\widehat{\mathcal{B}}_{\alpha}$. That is,

$$
\left(\exists \text { function } \widehat{h}_{\alpha}\right)\left[\widehat{h}_{\alpha}: \widehat{\mathcal{B}}_{\alpha} \rightarrow\left(\widehat{\mathcal{M}}_{\alpha}-\widehat{\mathcal{B}}_{\alpha}\right) \&\left(\forall \widehat{\nu} \in \widehat{\mathcal{B}}_{\alpha}\right)\left[\widehat{\nu}<_{B} \widehat{h}_{\alpha}(\widehat{\nu})\right]\right] .
$$

It is Blue's responsibility to move any $\widehat{x} \in \widehat{R}_{\alpha}$ for which $\nu(\alpha, \hat{x}, s)=\hat{\nu}_{0} \in \widehat{\mathcal{B}}_{\alpha}$ to the target state $\hat{\nu}_{1}=\widehat{h}_{\alpha}\left(\hat{\nu}_{0}\right)$ (and where $\widehat{h}$ is called the target function) so that Blue can achieve

$$
\left(\forall \widehat{x} \in \widehat{R}_{\alpha}\right)(\forall s)\left[\nu(\alpha, \hat{x}, s) \in \widehat{\mathcal{B}}_{\alpha} \Longrightarrow(\exists t>s)\left[\nu(\alpha, \hat{x}, t) \in \widehat{\mathcal{M}}_{\alpha}-\widehat{\mathcal{B}}_{\alpha}\right]\right],
$$

and hence Blue will cause every state $\hat{\nu}_{0} \in \widehat{\mathcal{B}}_{\alpha}$ to be emptied. To achieve (54) on $\widehat{R}_{\alpha}$ it suffices to achieve the following on $\widehat{S}_{\gamma}$ for each $\gamma \succeq \alpha$,

$$
\left(\forall \widehat{x} \in \widehat{S}_{\gamma}\right)(\forall s)\left[\nu(\gamma, \hat{x}, s) \in \widehat{\mathcal{B}}_{\gamma} \Longrightarrow(\exists t>s)\left[\nu(\gamma, \hat{x}, t) \in \widehat{\mathcal{M}}_{\gamma}-\widehat{\mathcal{B}}_{\gamma}\right]\right] .
$$

We often refer to the dual of (53), which asserts

$$
\left(\exists \text { function } h_{\alpha}\right)\left[h_{\alpha}: \mathcal{B}_{\alpha} \rightarrow\left(\mathcal{M}_{\alpha}-\mathcal{B}_{\alpha}\right) \&\left(\forall \nu \in \mathcal{B}_{\alpha}\right)\left[\nu<_{B} h_{\alpha}(\nu)\right]\right],
$$

and which enables us to achieve the dual of (55),

$$
\left(\forall x \in S_{\gamma}\right)(\forall s)\left[\nu(\gamma, x, s) \in \mathcal{B}_{\gamma} \Longrightarrow(\exists t>s)\left[\nu(\gamma, x, t) \in \mathcal{M}_{\gamma}-\mathcal{B}_{\gamma}\right]\right]
$$

Finally, we have ensured, for all $\gamma \prec f$ and all $\nu_{0} \in \mathcal{M}_{\gamma}$,

$$
\begin{gathered}
(\exists<\infty x)\left[x \in Y_{\gamma} \& \nu(\gamma, x)=\nu_{0}\right] \Longrightarrow \\
(\exists \alpha)_{\gamma \prec \alpha \prec f}\left[\left\{\nu_{1} \in \mathcal{M}_{\alpha}: \nu_{1}\left\lceil\gamma=\nu_{0}\right\} \subseteq \mathcal{R}_{\alpha} \cup \mathcal{B}_{\alpha}\right] .\right.
\end{gathered}
$$

To check (58) fix $\gamma \prec f$ and $\nu_{0} \in \mathcal{M}_{\gamma}$. By (27) $Y_{\gamma}={ }^{*} \omega$, so if the hypothesis of (58) holds, then we can choose $b$ such that

$$
(\forall x \in \omega)\left[x>b \Longrightarrow \nu(\gamma, x) \neq \nu_{0}\right]
$$


Choose $\alpha \prec f$ such that $\alpha \succ \gamma,|\alpha|>b$ and $|\alpha| \equiv 3 \bmod 5$. Consider any $\nu_{1} \in \mathcal{M}_{\alpha}$ such that $\nu_{1}\left\lceil\gamma=\nu_{0}\right.$. If $\nu_{1} \notin \mathcal{R}_{\alpha}^{<\alpha} \cup \mathcal{B}_{\alpha}^{<\alpha}$, then $F\left(\alpha^{-}, \nu_{1}\right)$ holds, so $\nu_{1} \in \mathcal{R}_{\alpha}^{\alpha}$ by (44), and hence $\nu_{1} \in \mathcal{R}_{\alpha}$ by (40).

Equations (45), (49), (54), (58) and their duals guarantee (39), as long as the well-visited $\alpha$-states are equal as well.

5.5. Verifying the automorphism requirement. We shall arrange that $\lim _{\alpha \prec f} \quad e_{\alpha}=\infty$. By (28) and (29) the sets $\left\{U_{\alpha}\right\}_{\alpha \prec f}$ and $\left\{V_{\alpha}\right\}_{\alpha \prec f}$ constitute skeletons for $\left\{W_{n}\right\}_{n \in \omega}$. By (35), its dual, and (36) we know that the well-visited $\alpha$-states on the $\omega$-side and $\widehat{\omega}$-side coincide. By (39) the well-resided $\alpha$-states also coincide, so (25) is satisfied. The construction in 87 and verification in 88 will demonstrate that the equations of $\$ 5.2, \$ 5.3$ and $\$ 5.4$ are satisfied. First we need a few more definitions.

\section{The TREe AND THE TRUE PATH}

In this section, we define the tree of strategies and the true path $f$ through the tree.

6.1. The well-visited states and $\mathcal{F}_{\beta}^{+}$. In 4.4 we said that every $\alpha \in T$ would have an associated set $\mathcal{M}_{\alpha}$ such that $\mathcal{M}_{\alpha}=\mathcal{F}_{\alpha}$ if $\alpha \prec f$. However, although this is the property we want $\mathcal{M}_{\alpha}$ to have, we cannot simply define $\mathcal{M}_{\alpha}$ to be $\alpha$ 's guess at $\mathcal{F}_{\alpha}$ because that definition would be circular. Rather we must define here a certain set $\mathcal{F}_{\beta}^{+}$which depends only on $\beta$, and then let $\mathcal{M}_{\alpha}$ be $\alpha$ 's guess at $\mathcal{F}_{\beta}^{+}$so that $\mathcal{M}_{\alpha}=\mathcal{F}_{\beta}^{+}\left(=\mathcal{F}_{\alpha}\right)$ for $\alpha \prec f$.

Fix $\alpha \in T$ such that $e_{\alpha}>e_{\beta}$ for $\beta=\alpha^{-}$. Define the c.e. set $Z_{e_{\alpha}}=\bigcup_{s} Z_{e_{\alpha}, s}$, where

$$
Z_{e_{\alpha}, s+1}:=\left\{x: x \in U_{e_{\alpha}, s+1} \& x \in Y_{\beta, s}\right\} .
$$

Define the $\alpha$-state function $\nu^{+}(\alpha, x, s)$ exactly as for $\nu(\alpha, x, s)$ in Definition 5.4 but with $Z_{e_{\alpha}, s}$ in place of $U_{\alpha, s}$.

Define

(61) $\mathcal{F}_{\beta}^{\bar{A}+}=\left\{\nu:\left(\exists^{\infty} x\right)(\exists s)\left[x \in Y_{\beta, s}^{\bar{A}}, \nu^{+}(\alpha, x, s)=\nu, 0 \notin \nu\right.\right.$, and $\left.\left.\widehat{\Psi}_{\alpha}(x)[s]=0\right]\right\}$ and

$$
\mathcal{F}_{\beta}^{A+}=\left\{\nu:\left(\exists^{\infty} x\right)(\exists s)\left[x \in Y_{\beta, s}^{A} \& \quad \nu^{+}(\alpha, x, s)=\nu\right]\right\} .
$$

Let $\mathcal{F}_{\beta}^{+}=\mathcal{F}_{\beta}^{\bar{A}+} \cup \mathcal{F}_{\beta}^{A+}$. Define

$$
k_{\beta}^{+}=\min \left\{y:(\forall x>y)(\forall s)\left[\left[x \in Y_{\beta, s} \quad \& \quad \nu^{+}(\alpha, x, s)=\nu_{1}\right] \Longrightarrow \nu_{1} \in \mathcal{F}_{\beta}^{+}\right]\right\} .
$$

If $e_{\alpha}>e_{\beta}$ we also define $\widehat{\mathcal{F}}_{\beta}^{+}=\left\{\widehat{\nu}: \nu \in \mathcal{F}_{\beta}^{+}\right\}$. (Note that $Z_{e_{\alpha}}$ and hence $\mathcal{F}_{\beta}^{+}$ and $k_{\beta}^{+}$depend only upon $\beta$ not $\alpha$ and thus $\alpha$ can make guesses $\mathcal{M}_{\alpha}$ and $k_{\alpha}$ for $\mathcal{F}_{\beta}^{+}$and $k_{\beta}^{+}$.)

If $\widehat{e}_{\alpha}>\widehat{e}_{\beta}$ we first define

$$
\widehat{\mathcal{F}}_{\beta}^{+}=\left\{\nu:\left(\exists^{\infty} x\right)(\exists s)\left[x \in Y_{\beta, s} \quad \& \quad \nu^{+}(\alpha, x, s)=\nu\right]\right\} .
$$

Define $k_{\beta}^{+}$using the dual of (63). Now we can define $\mathcal{F}_{\beta}^{+}=\left\{\nu: \widehat{\nu} \in \mathcal{F}_{\beta}^{+}\right\}$. (Note that there is no $\widehat{k}_{\beta}^{+}$only $k_{\beta}^{+}$.) 
Every $\alpha \in T$ will have associated items $\mathcal{M}_{\alpha}$ and $k_{\alpha}$ such that $\mathcal{M}_{\alpha}=\mathcal{F}_{\beta}^{+}$and $k_{\alpha}=k_{\beta}^{+}$for $\alpha \prec f$. We allow $x$ to enter $Y_{\alpha}$ only if $x>k_{\alpha}$. If $e_{\alpha}=e_{\beta}$ and $\widehat{e}_{\alpha}=\widehat{e}_{\beta}$ we define $\mathcal{F}_{\beta}^{+}=\mathcal{F}_{\beta}, \widehat{\mathcal{F}}_{\beta}^{+}=\widehat{\mathcal{F}}_{\beta}$, and $k_{\beta}^{+}=k_{\beta}$. If

$$
(\exists x)(\exists s)\left[x \in Y_{\alpha, s} \quad \& \quad \nu(\alpha, x, s) \notin \mathcal{M}_{\alpha}\right],
$$

then we say that $\alpha$ is provably incorrect at all stages $t \geq s$ and we ensure that $\alpha \nprec f$.

\subsection{The definition of the tree $T$.}

Definition 6.1. We say that $\alpha \in T$ is consistent if $\alpha$ is $\mathcal{M}$-consistent (Definition 5.7) and $\mathcal{R}$-consistent (Definition [5.8).

Note that by clause (i) in the following Definition 6.2 of $T$,

$$
\beta \in T \Longrightarrow[\beta \text { inconsistent } \Longleftrightarrow \beta \text { is a terminal node on } T] \text {. }
$$

We shall show that if $\alpha \prec f$, then $\alpha$ is consistent, and therefore $\lim _{\alpha \prec f} e_{\alpha}=\infty$, so the argument of $\$ 5.5$ applies.

Definition 6.2. Put $\lambda \in T$ and define $\mathcal{M}_{\lambda}=\mathcal{R}_{\lambda}=\mathcal{B}_{\lambda}=\langle\lambda, \emptyset, \emptyset\rangle$, and $k_{\lambda}=e_{\lambda}=$ $\widehat{e}_{\lambda}=-1$. If $\beta \in T$ we put $\alpha=\beta \wedge\left\langle\mathcal{M}_{\alpha}, \mathcal{R}_{\alpha}, \mathcal{B}_{\alpha}, k_{\alpha}\right\rangle$ in $T$ providing the following conditions hold:

(i) $\beta$ is consistent (as defined in Definition 6.1),

(ii) $\mathcal{M}_{\alpha}$ is a set of $\alpha$-states, $\mathcal{R}_{\alpha} \subseteq \mathcal{M}_{\alpha}, \mathcal{B}_{\alpha} \subseteq \mathcal{M}_{\alpha}$, and $\mathcal{R}_{\alpha} \cap \mathcal{B}_{\alpha}=\emptyset$,

(iii) $\left.\mathcal{M}_{\alpha}\right\rceil \beta \subseteq \mathcal{M}_{\beta}$,

(iv) $\left[e_{\alpha}=e_{\beta} \& \widehat{e}_{\alpha}=\widehat{e}_{\beta}\right] \Longrightarrow \mathcal{M}_{\alpha}=\mathcal{M}_{\beta} \quad \& \quad k_{\alpha}=k_{\beta}$,

(v) $\mathcal{R}_{\alpha}^{<\alpha}:=\left\{\nu \in \mathcal{M}_{\alpha}: \nu \uparrow \beta \in \mathcal{R}_{\beta}\right\} \subseteq \mathcal{R}_{\alpha}$,

(vi) $\mathcal{B}_{\alpha}^{<\alpha}:=\left\{\nu \in \mathcal{M}_{\alpha}: \nu \uparrow \beta \in \mathcal{B}_{\beta}\right\} \subseteq \mathcal{B}_{\alpha}$,

(vii) $\quad \mathcal{R}_{\alpha}^{\alpha}:=\mathcal{R}_{\alpha}-\mathcal{R}_{\alpha}^{<\alpha} \neq \emptyset \Longrightarrow|\alpha| \equiv 3 \bmod 5$,

(viii) $\quad \mathcal{B}_{\alpha}^{\alpha}:=\mathcal{B}_{\alpha}-\mathcal{B}_{\alpha}^{<\alpha} \neq \emptyset \Longrightarrow|\alpha| \equiv 4 \bmod 5$,

(ix) $|\alpha| \equiv 0 \bmod 5 \Longrightarrow\left\langle\mathcal{M}_{\alpha}, \mathcal{R}_{\alpha}, \mathcal{B}_{\alpha}, k_{\alpha}\right\rangle=\left\langle\mathcal{M}_{\beta}, \mathcal{R}_{\beta}, \mathcal{B}_{\beta}, k_{\beta}\right\rangle$. These nodes correspond to the positive requirement, so they are also associated with either a 0 representing the $\Pi_{2}$-outcome, or any $p \in \omega, p>0$, representing the $\Sigma_{2}$-outcomes. These latter have the $\omega^{*}$-ordering, with 0 the leftmost node and 1 the rightmost.

In addition, each $\alpha \in T$ has associated dual sets $\widehat{\mathcal{M}}_{\alpha}, \widehat{\mathcal{R}}_{\alpha}$, and $\widehat{\mathcal{B}}_{\alpha}$, which are determined from $\mathcal{M}_{\alpha}, \mathcal{B}_{\alpha}$, and $\mathcal{R}_{\alpha}$, by (36), (46), and (45), respectively. Also $\alpha$ has associated integers $e_{\alpha}$ and $\widehat{e}_{\alpha}$ (depending only on $\left.|\alpha|\right)$ as defined at the beginning of 95.3 . (We identify the finite object $\left\langle\mathcal{M}_{\alpha}, \mathcal{R}_{\alpha}, \mathcal{B}_{\alpha}, k_{\alpha}\right\rangle$ with an integer under some effective coding, so we may regard $T \subseteq \omega^{<\omega}$.) For $\mathcal{M}_{\alpha} \supset \mathcal{M}_{\alpha^{\prime}}$, where $|\alpha|=\left|\alpha^{\prime}\right|$, we order the nodes so that $\alpha<_{L} \alpha^{\prime}$ and similarly for $\mathcal{R}_{\alpha}$ and $\mathcal{B}_{\alpha}$. We also ensure that if $k_{\alpha}<k_{\alpha}^{\prime}$, then $\alpha<_{L} \alpha^{\prime}$.

Definition 6.3. The true path $f \in[T]$ is defined by induction on $n$. We define $f\left\lceil 1\right.$ to be the node $\rho$ of length 1 such that $\mathcal{M}_{\rho}$ contains all possible $\rho$-states and $k_{\rho}=0$. Note there are exactly two states in $\mathcal{M}_{\rho}$, the state containing $U_{\rho}={ }^{*} A$ and the state that does not contain $U_{\rho}$. For $\widehat{\mathcal{M}}_{\alpha}$, the two states correspond to the one containing $\widehat{U}_{\rho}=B$ and the one that does not. When we refer to $B_{s}$, we are actually referring to $\widehat{U}_{\rho, s}$. The approximation of the true path $f_{s}$ will go through $\rho$ for all $s$. 
Let $\beta=f\left\lceil n\right.$ be consistent. Then $f \uparrow(n+1)$ is the $<_{L}$-least $\alpha \in T, \alpha \succ \beta$, of length $m=n+1$ such that:

(i) For $m=5 e, f(n)=0$ if $\beta$ appoints infinitely many witnesses, and $f(n)=$ $p+1$ if $\beta$ enumerates $p$ witnesses into $D$,

(ii) $m \equiv 1 \bmod 5 \Longrightarrow \mathcal{M}_{\alpha}=\mathcal{F}_{\beta}^{+} \& k_{\alpha}=k_{\beta}^{+}$,

(iii) $m \equiv 2 \bmod 5 \Longrightarrow \widehat{\mathcal{M}}_{\alpha}=\widehat{\mathcal{F}}_{\beta}^{+} \quad \& \quad k_{\alpha}=k_{\beta}^{+}$,

(iv)

$$
m \equiv 3 \bmod 5 \Longrightarrow \begin{gathered}
\mathcal{R}_{\alpha}^{\alpha}=\left\{\nu: \nu \in \mathcal{M}_{\alpha}-\left(\mathcal{R}_{\alpha}^{<\alpha} \cap \mathcal{B}_{\alpha}^{<\alpha}\right) \& F(\beta, \nu)\right\} \\
\& \widehat{\mathcal{B}}_{\alpha}^{\alpha}=\left\{\widehat{\nu}: \nu \in \mathcal{R}_{\alpha}^{\alpha}\right\},
\end{gathered}
$$

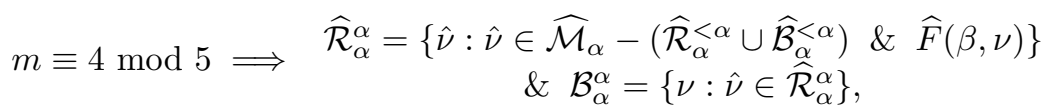

(vi) unless otherwise specified in (i)-(iv), $\mathcal{M}_{\alpha}, \mathcal{R}_{\alpha}, \mathcal{B}_{\alpha}$, and $k_{\alpha}$ take the values $\mathcal{M}_{\beta}, \mathcal{R}_{\beta}, \mathcal{B}_{\beta}$, and $k_{\beta}$, respectively.

For a consistent $\beta=f\left\lceil n\right.$, note that $\mathcal{F}_{\beta}^{+}$is just a finite set of states and $k_{\beta}^{+}$is an integer, so clearly $\alpha$ exists. We shall prove that if $\alpha \prec f$, then $\alpha$ is consistent, so the true path $f$ exists and is infinite. Note that each of the conditions in Definition 6.3 is $\Pi_{2}^{0}$. A computable approximation to the true path, the limit inferior of which will be the true path itself, is given along with the $D$-Module in the next section.

\section{The CONSTRUCTION}

Let $T$ be the tree defined in $\$ 6.2$. We simultaneously construct $D$ and the sets $U_{\alpha}, \widehat{U}_{\alpha}, V_{\alpha}, \widehat{V}_{\alpha}$. At each stage we also define a function $f_{s} \in T,\left|f_{s}\right|=2 s$, such that $f=\liminf _{s} f_{s}$. We say that stage $s$ is an $\alpha$-stage if $\alpha \prec f_{s}$.

7.1. Order of the construction. We can think of our construction happening on four identical trees, corresponding to $\bar{A}, \bar{B}, A$, and $B$. Every $x \in \omega$ begins on the $\bar{A}$ tree and moves through the nodes. If $x \in A$, it will eventually move from the $\bar{A}$ tree to the $A$ tree. This happens for each $\hat{x} \in \hat{\omega}$ for the $B$ side as well.

In the following construction, Steps 1-5 are for the $\bar{A}$ tree. For the $A$ tree, the Step 1-5 construction is exactly the construction in the Harrington-Soare paper, except without distinguishing between $S^{0}$ and $S^{1}$. Similarly, Steps $\widehat{1}-\widehat{5}$, which apply to the $\bar{B}$ and $B$ trees are also as in the Harrington-Soare construction in [4, except with the addition of switching elements from the $\bar{B}$ tree to the $B$ tree. These steps are identical (or dual) to the Steps 1-5 mentioned here, except that any mention of keeping elements out of $A$, such as in (1.8), is omitted.

At the beginning of each stage $s$, we perform one of Steps 1-5 of the $A$ or $\bar{A}$ tree construction if any applies. If no step can act, we perform one of Steps $\widehat{1}-\widehat{5}$ for the $B$ and $\bar{B}$ trees. Regardless of whether we performed any of these steps, we continue to Step B and the $D$-Module. If none of the Steps $1-5$ or $\widehat{1}-\widehat{5}$ has acted on any of the four trees, we proceed to Step 6 after performing Step B and the $D$-Module. Step 6 applies to all four trees. 
7.2. The automorphism construction. We perform the automorphism construction as in the $\Delta_{3}^{0}$ paper, with some important changes. We switch elements $x$ from the $\bar{A}$ tree to the $A$ tree under Step 4 when they enter $A=W_{i}$. Let $U_{0}=A=W_{i}$.

To initialize node $\alpha$ means: to remove every $x \in S_{\alpha, s}\left(\widehat{x} \in \widehat{S}_{\alpha, s}\right)$, and put $x$ in $S_{\beta}\left(\widehat{x}\right.$ in $\left.\widehat{S}_{\beta}\right)$ for $\beta=\alpha \cap f_{s+1}$ (where $\alpha \cap \delta$ denotes the longest $\gamma$ such that $\gamma \preceq \alpha$ and $\gamma \preceq \delta)$.

We present in the next section Steps $1-5$ for the construction and a final Step 6 at which we define $f_{s+1}$. These properties will produce the automorphism.

Besides adding the $D$ and $B$ constructions, we must also make some changes to the old automorphism construction. The primary difference between this construction and the Harrington-Soare construction of 4 is that we now must only allow elements to move in the $\bar{A}$ tree if they appear to be in $\bar{A}$. Each of Steps 1-5 now requires that the element in question $x$ must satisfy the condition that $\widehat{\Psi}^{D}(x)[s]=0$. This condition says that $D$ believes $x$ is in $\bar{A}$. This condition is actually only important for Step 1, but we must impose it on all steps because otherwise, another step, such as Step 3, could empty out a state before Step 1 has the ability to pull elements from that state down to the next node. By requiring that all steps wait for elements to appear to be in $\bar{A}$, Step 1 will have priority over the other steps.

Note that in order to ensure that Step 3 can act, we allow it to go immediately after Step 1, so that elements pulled into $S_{\alpha}$ by Step 1 will still appear to be in $\bar{A}$ when Step 3 acts. Otherwise, Step 3 may never have the ability to act. We only allow Step 3 to act in this way for half of the elements pulled into a state by Step 1, ensuring that the state is not prematurely emptied.

7.3. The steps of the construction. Stage $s=0$. For all $\alpha \in T$ define $U_{\alpha, 0}=$ $V_{\alpha, 0}=\widehat{U}_{\alpha, 0}=\widehat{V}_{\alpha, 0}=\emptyset$, and define $m(\alpha, 0)=0$. Define $Y_{\lambda, 0}=\widehat{Y}_{\lambda, 0}=\emptyset$, and $f_{0}=\lambda$.

Let $B_{0}=\emptyset$, and define $\delta_{0}=\lambda$, the empty node on $T$.

Stage $s+1$. Find the least $n<6$ such that Step $n$ applies to some $x \in Y_{\alpha, s}$, and perform the indicated action. This can apply to either the $A$ or $\bar{A}$ trees. If there is no such $n$, then likewise find the least $n<6$ such that Step $\widehat{n}$ applies to some $\widehat{x} \in \widehat{Y}_{\alpha, s}$, and perform the indicated action. This can apply to either the $B$ or $\bar{B}$ trees.

Next, check if Step B applies, and perform the indicated action if it does.

Then perform the $D$-Module. Note that both Step B and the $D$-Module should be performed after Steps $1-5$ and Steps $\widehat{1}-\widehat{5}$, regardless of whether these steps actually acted.

If none of Steps $1-5$ or Steps $\widehat{1}-\widehat{5}$ acted, then perform Step 6 after Step B and the $D$-Module. (It is important that these steps be performed in the indicated order.)

7.4. Steps 1-5. Step 1. (Prompt pulling of $x$ from $R_{\beta}$ to $S_{\alpha}$ to ensure $\mathcal{M}_{\alpha} \subseteq \mathcal{E}_{\alpha}$ ) Suppose $\left\langle\alpha, \nu_{i}\right\rangle$ is the first unmarked entry on the list $\mathcal{L}_{s}$ defined in Step 6 such that the following conditions hold for some $x$, where $\nu_{i}=\left\langle\alpha, \sigma_{i}, \tau_{i}\right\rangle$, and $A \notin \sigma_{i}$ unless $e_{\alpha}=0$.

(1.1) $x \in R_{\beta, s}-Y_{\alpha, s}$,

(1.2) $x>k_{\alpha}, x>|\alpha|$, and $x>p$ for $\alpha=\beta^{\wedge} p$ and $|\alpha|=5 e$, 
(1.3) $x$ is $\alpha$-eligible (i.e., $\neg(\exists t)\left[x \leq t \leq s \quad \& \quad f_{t}<\alpha\right]$ ),

(1.4) $\neg\left[\alpha(x, s)<_{L} \alpha\right]$,

(1.5) $x>m(\alpha, s)$,

(1.6) $\nu(\beta, x, s)=\nu_{i} \uparrow \beta$,

(1.7) $e_{\alpha}>e_{\beta} \Longrightarrow \nu^{+}(\alpha, x, s)=\nu_{i}$,

(1.8) if $A \notin \sigma_{i}, \widehat{\Psi}_{\alpha}(x)[s] \downarrow=0$.

Action. Choose the least $x$ corresponding to $\left\langle\alpha, \nu_{i}\right\rangle$, and do the following.

(1.9) Mark the $\alpha$-entry $\left\langle\alpha, \nu_{i}\right\rangle$ on $\mathcal{L}_{s}$, and suppose this is the $k^{\text {th }}$ occurrence of $\left\langle\alpha, \nu_{i}\right\rangle$ on $\mathcal{L}_{s}$.

(1.10) Move $x$ to $S_{\alpha}$.

(1.11) If $e_{\alpha}>e_{\beta}$ and $e_{\alpha} \in \sigma_{i}$, then enumerate $x$ in $U_{\alpha, s+1}$.

(1.12) If $\widehat{e}_{\alpha}>\widehat{e}_{\beta}$ and $\widehat{e}_{\alpha} \in \tau_{i}$, then enumerate $x$ in $\widehat{V}_{\alpha, s+1}$. (Hence, $\nu(\alpha, x, s+1)$

$=\nu_{i}$. Also $\nu_{i} \in \mathcal{M}_{\alpha}$ because $\left\langle\alpha, \nu_{i}\right\rangle \in \mathcal{L}$ implies $\nu_{i} \in \mathcal{M}_{\alpha}$.)

(1.13) If $k$ is odd and $A \notin \sigma_{i}$, immediately perform Step 3, if it applies.

(1.14) If $A \in \sigma_{i}$, switch $x$ to the $A$ tree, and enumerate $\langle\alpha, x, i\rangle$ in $\Lambda$.

Step 2. (Move $x$ from $S_{\beta}$ to $S_{\alpha}$, so $Y_{\alpha}={ }^{*} \omega$ ) Suppose there is an $x$ such that

(2.1) $x \in S_{\beta, s}$,

(2.2) $x>|\alpha|$ and $x>k_{\alpha}$, and $x>p$ for $\alpha=\beta^{\wedge} p$ and $|\alpha|=5 e$,

(2.3) $x$ is $\alpha$-eligible,

(2.4) $x<m(\alpha, s)$,

(2.5) $\widehat{\Psi}_{\alpha}(x)[s] \downarrow=0$,

(2.6) $\alpha$ is the $<_{L}$-least $\gamma \in T$ with $\gamma^{-}=\beta$ satisfying (2.1)-(2.5).

Action. Choose the least pair $\langle\alpha, x\rangle$ and

(2.7) move $x$ from $S_{\beta}$ to $S_{\alpha}$.

(In Step 2 we need (2.4), so $Y_{\alpha}$ will not grow while $\alpha$ is waiting for another prompt pulling under Step 1.)

Step 3. (For $\alpha \mathcal{M}$-inconsistent to ensure $\alpha \nprec f$ ) Suppose for $\alpha \in T$ there exists $x$ such that,

(3.1) $e_{\alpha}>e_{\beta}$,

(3.2) $x \in S_{\alpha, s}$,

(3.3) $\nu(\alpha, x, s)=\nu_{0} \in \mathcal{M}_{\alpha}$,

(3.4) $\left(\exists \nu_{1}\right)\left[\nu_{0}<_{\mathrm{B}} \nu_{1} \quad \& \quad \nu_{1}\left\lceil\beta \in \mathcal{M}_{\beta} \quad \& \quad \nu_{1} \notin \mathcal{M}_{\alpha}\right]\right.$,

(3.5) $\widehat{\Psi}_{\alpha}(x)[s] \downarrow=0$.

Action. Choose the least such pair $\langle\alpha, x\rangle$ and

(3.6) enumerate $x$ in $\widehat{V}_{\delta, s+1}$ for all $\delta \prec \alpha$ such that $e_{\delta} \in \tau_{1}$. (This action causes $\nu(\alpha, x, s+1)=\nu_{1}$. Hence, $\alpha$ is provably incorrect at all stages $t \geq s+1$ so $\alpha \nprec f$.)

Step 4. (Delayed Red enumeration into $U_{\alpha}$ ) Suppose $x \in R_{\alpha, s}$, or $x \in S_{\lambda, s}$ and let $\alpha=\rho$, and
(4.1) $e_{\alpha}>e_{\beta}$,
(4.2) $x \notin U_{\alpha, s}$,
(4.3) $x \in Z_{e_{\alpha}, s}:=U_{e_{\alpha}, s} \cap Y_{\beta, s-1}$, and
(4.3) if $\alpha \neq \rho$, then $\widehat{\Psi}_{\alpha}(x)[s] \downarrow=0$. 
Action. Choose the least such pair $\langle\alpha, x\rangle$ and

(4.5) enumerate $x$ in $U_{\alpha, s+1}$.

(4.6) If $e_{\alpha}=0$, switch $x$ to the $A$ tree, and enumerate $\langle\gamma, x, i\rangle$ in the list $\Lambda$ for $x \in S_{\gamma}, s$ for $\nu(\gamma, x, s)=\nu_{i}$.

Step 5. (Blue emptying of state $\nu \in \mathcal{B}_{\alpha}$ ) Suppose for $\alpha \in T$ there exists $x$ such that either Case 1 or Case 2 holds.

Case 1. Suppose

(5.1) $\nu(\alpha, x, s)=\nu_{0} \in \mathcal{B}_{\alpha}$, say $\nu_{0}=\left\langle\alpha, \sigma_{0}, \tau_{0}\right\rangle$,

(5.2) $x \in S_{\alpha, s}$,

(5.3) $\alpha$ is $\mathcal{M}$-consistent and $\mathcal{R}$-consistent,

(5.4) $\widehat{\Psi}_{\alpha}(x)[s] \downarrow=0$.

Action. Choose the least such pair $\langle\alpha, x\rangle$. Let $\nu_{1}=h_{\alpha}\left(\nu_{0}\right)>_{B} \nu_{0}$, where $h_{\alpha}$ is a target function satisfying (56). Let $\nu_{1}=\left\langle\alpha, \sigma_{1}, \tau_{1}\right\rangle$.

(5.5) Enumerate $x \in \widehat{V}_{\delta}$ for all $\delta \preceq \alpha$ such that $\widehat{e}_{\delta}>\widehat{e}_{\delta^{-}}$and also $e_{\delta} \in \tau_{1}-\tau_{0}$. (Hence, $\nu(\alpha, x, s+1)=\nu_{1}$.)

Case 2. Suppose that (5.1) holds and

(5.6) $x \in S_{\gamma, s}$ where $\gamma^{-}=\alpha$, and

(5.6) $\gamma$ is either $\mathcal{M}$-inconsistent or $\mathcal{R}$-inconsistent,

(5.7) $\widehat{\Psi}_{\alpha}(x)[s] \downarrow=0$.

Action. Perform the same action as in Case 1 to achieve $\nu(\alpha, x, s+1)=\nu_{1}$.

(In (5.6) note that by (66) $\gamma \in T$ implies (5.3) for $\alpha=\gamma^{-}$, so $h_{\alpha}$ exists in Case 2. Note in Step 5, Case 2 that the enumeration may not be $\gamma$-legal, i.e., perhaps $\nu(\gamma, x, s+1) \notin \mathcal{M}_{\gamma}$, but this will not matter because we shall prove that $\gamma \nprec f$ if $\gamma$ is inconsistent. Hence, it only matters that the enumeration is $\alpha$-legal, i.e., $\left.\nu(\alpha, x, s) \in \mathcal{M}_{\alpha}.\right)$

7.5. Step B and the D-Module. Step B. Let $\langle\alpha, x, e\rangle$ be the least entry on the list $\Lambda$ such that there is a $y$ that has never before caused action on this step, satisfying

(B.1) $y \in \widehat{S}_{\alpha}^{\bar{A}}$,

(B.2) $\widehat{\nu}(\alpha, y, s)=\widehat{\nu}_{e}$,

(B.3) for all $i<\langle\alpha, x, e\rangle, \varphi_{i}^{B}(i)[s] \downarrow \Longrightarrow \varphi_{i}^{B}(i)[s]<y$.

Action. (B.4) If $\langle\alpha, x, e\rangle$ is not checked, check $\langle\alpha, x, e\rangle$, and do not enumerate $y$ into $B$. If $\langle\alpha, x, e\rangle$ has been checked already, enumerate $y$ into $B$, switching $y$ to the $B$ tree, and remove $\langle\alpha, x, e\rangle$ from the list $\Lambda$. This will leave infinitely many elements in $\bar{B}$, while still matching the flow into $A$.

The $D$-Module (and approximation to the true path). Define $f_{s+1}$ by a series of substages $t, 0 \leq t \leq 2 s$, where we define $\delta^{t}$ at substage $t$, as follows, and later define $f_{s+1}=\delta^{2 s}$. (Note that in the full theorem as described in 4.7 . we must perform this for multiple different trees $T_{\alpha}$ at the same time to get the approximation to the true path.)

Substage $t, 0 \leq t \leq 2 s$. Define $\delta^{0}=\lambda$ and $\delta^{1}=\rho$ as in Definition 6.3. Given $\delta^{t}$, perform the following action. 
Case 1. $\left|\delta^{t}\right| \equiv 0$ (or 1) $\bmod 5$. Let $\beta=\delta^{t}$. Given $\delta^{t}$ let $v \leq s$ be maximal such that $\delta^{t} \preceq f_{v}$ if $v$ exists and let $v=0$ otherwise. For $\left|\delta^{t}\right| \equiv 0 \bmod 5$, let $\alpha$ be the leftmost node (i.e. the largest possible $\mathcal{M}_{\alpha}$ ) with $\alpha^{-}=\beta$ such that for all $\nu \in \mathcal{M}_{\alpha}^{\bar{A}}$,

$$
\begin{array}{r}
\left|\left\{x:(\exists t \leq s)\left[x \in Y_{\beta, t}^{\bar{A}}, \nu^{+}(\alpha, x, t)=\nu, 0 \notin \nu, \quad \& \widehat{\Psi}_{\alpha}(x)[t]=0\right]\right\}\right| \\
>\left|\left\{x:(\exists t \leq v)\left[x \in Y_{\beta, t}^{\bar{A}}, \nu^{+}(\alpha, x, t)=\nu, 0 \notin \nu, \quad \& \widehat{\Psi}_{\alpha}(x)[t]=0\right]\right\}\right|,
\end{array}
$$

for all $\nu \in \mathcal{M}_{\alpha}^{A}$,

$$
\begin{aligned}
\mid\left\{x:(\exists t \leq s)\left[x \in Y_{\beta, t}^{\bar{A}} \& \nu^{+}(\alpha, x, t)\right.\right. & =\nu]\} \mid \\
> & \left|\left\{x:(\exists t \leq v)\left[x \in Y_{\beta, t}^{\bar{A}} \& \nu^{+}(\alpha, x, t)=\nu\right]\right\}\right|,
\end{aligned}
$$

and

$$
k_{\alpha}=\begin{gathered}
(\mu u<s)\left(\forall s^{\prime}, u<s^{\prime}<s\right) \\
{\left[\left[x \in Y_{\beta, s^{\prime}} \& \nu^{+}\left(\alpha, x, s^{\prime}\right)=\nu_{1}\right] \Longrightarrow \nu_{1} \in \mathcal{M}_{\alpha}\right] .}
\end{gathered}
$$

Set $\delta^{t+1}=\alpha$. If no such $\alpha$ exists, let $\delta^{t+1}$ be the rightmost extension $\alpha$ of $\delta^{t}$ such that $k_{\alpha}=s$. The dual is similar, for $\left|\delta^{t}\right| \equiv 1 \bmod 5$, except that it uses only equations (68) and (69).

Case 2. $\left|\delta^{t}\right| \equiv 2($ or 3$) \bmod 5$. Let $\beta=\delta^{t}$. Given $\delta^{t}$ let $v \leq s$ be maximal such that $\delta^{t} \preceq f_{v}$ if $v$ exists and let $v=0$ otherwise. For each $\alpha$ with $\alpha^{-}=\beta$, let $g(\alpha, s)$ be the greatest $h<s$ such that for all $x \leq h, \nu(\beta, x, s) \notin \mathcal{R}_{\alpha}^{\alpha}$. Let $\delta^{t+1}$ be the leftmost $\alpha$ such that $g(\alpha, s)>g(\alpha, v)$. If no such $\alpha$ exists, let $\delta^{t+1}$ be the rightmost extension of $\beta$ of length $\left|\delta^{t}\right|+1$. The dual for $\left|\delta^{t}\right| \equiv 3 \bmod 5$ is similar.

Case 3. $\left|\delta^{t}\right| \equiv 4 \bmod 5$. Let $\beta=\delta^{t}$. Here $\beta$ is associated with the strategy for $P_{j}$ described in 3.2 . These correspond to what we called odd nodes in the construction of a nonlow low 2 set. (In particular, we assume a set $Z_{\beta}$ is being defined whose index $g(\beta)$ is known a priori, such that $W_{g(\beta)}=Z_{\beta}, g$ is the computable function as in 3.2 Step 1a but adjusted with $\beta$ in place of $j$, and that our objective is to arrange that either $\lim _{s} \widetilde{\varphi}_{j, s}(g(\beta)) \uparrow$, or gives the wrong answer about whether $W_{g(\beta)} \cap \bar{D} \neq \emptyset$.)

Subcase 1. (Like 33.2 Step 1a) Suppose $Z_{\beta, s} \cap \bar{D}_{s}=\emptyset, \widetilde{\varphi}_{j, s}(g(\beta)) \downarrow=0$, and there is no $\beta$-witness in $\bar{D}_{s}$. Let the $\beta$-witnesses previously appointed be $x_{1}^{\beta} \ldots x_{n-1}^{\beta}$. Suppose

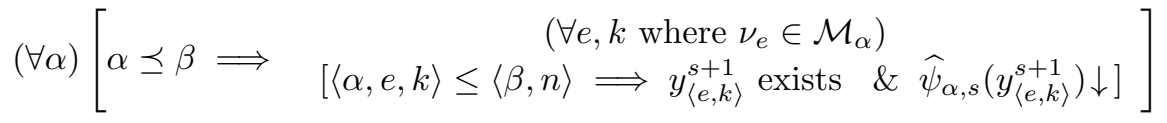

and for all $\xi$ with $|\xi| \equiv 4 \bmod 5$,

$$
\begin{array}{r}
\xi^{\prec} p \prec \beta \quad \Longrightarrow \quad x_{n^{\prime}}^{\xi} \in D_{s} \text { for all } n^{\prime}<p, \\
\text { and } \xi^{\prec} 0 \prec \beta \&\left\langle\xi, n^{\prime}\right\rangle<\langle\beta, n\rangle \quad \Longrightarrow \quad x_{n^{\prime}}^{\xi} \in D_{s} .
\end{array}
$$

Define $y$ to be the smallest $z \in \bar{D}_{s} \cap \omega^{[\beta]}$, where $\beta$ is identified with its Gödel number, such that $\Gamma_{s}^{\beta}<z<\Lambda_{s}^{\beta}$, and

$$
(\forall \alpha \preceq \beta)\left[\left(\forall e, k \text { with } \nu_{e} \in \mathcal{M}_{\alpha} \&\langle\alpha, e, k\rangle<\langle\beta, n\rangle\right)\left[\widehat{\psi}_{\alpha, s}\left(y_{\langle e, k\rangle}\right) \downarrow<z\right]\right]
$$
and $\left(\forall \gamma<_{L} \beta\right)[z$ is greater than any current witness at $\gamma]$. 
If $y$ exists, then appoint $y$ as the next $\beta$-witness, call it $x_{n}^{\beta}$, define $\Gamma_{s+1}^{\beta}=x_{n}^{\beta}$, and enumerate $x_{n}^{\beta}$ in $Z_{\beta, s+1}$.

Subcase 2. (Like 33.2 Step 1b) Suppose that $\widetilde{\varphi}_{j, s}(g(\beta)) \downarrow=1, \Gamma_{s}^{\beta}$ is defined and equal to some $\beta$-witness $x_{n}^{\beta}$, and that for all $\xi$ with $|\xi| \equiv 4 \bmod 5$,

$$
\xi^{\prec} 0 \prec \beta \Longrightarrow \Gamma_{s}^{\xi}=x_{p}^{\xi} \in D_{s+1} .
$$

Then enumerate $x_{n}^{\beta}$ in $D_{s+1}$, and define

$$
\Gamma_{s+1}^{\beta}=\max \left\{\Gamma_{s}^{\beta}, s+1\right\} .
$$

(Equation (73) says that for every $\xi$ with $|\xi| \equiv \bmod 5$ and $\xi^{\prec} 0 \prec \beta$, i.e., for which $\beta$ is guessing that $\xi$ has the $\Pi_{2}$-outcome of infinitely many witnesses, $\beta$ must wait until $\xi$ actually puts its witness $\Gamma_{s}=x_{p}^{\xi}$ into $D_{s+1}$ during an earlier substage of stage $s+1$ before $\beta$ is allowed to simultaneously contribute its own witness at a later substage of stage $s+1$.)

Subcase 3. Otherwise. If $\Gamma_{s}^{\beta}=x_{n}^{\beta}$ for some $\beta$-witness $x_{n}^{\beta}$, then define $\Gamma_{s+1}^{\beta}=\Gamma_{s}^{\beta}$, and if not, then define $\Gamma_{s+1}^{\beta}=\max \left\{\Gamma_{s}^{\beta}, s+1\right\}$.

To complete Case 3 define $\delta^{t+1}=\delta^{t \frown} 0$ if Subcase 2 holds, and $\delta^{t+1}=\delta^{t \frown} p+1$, otherwise, where $p$ is the number of $\beta$-witnesses that have been enumerated into $D$. Complete the construction by defining $f_{s+1}=\delta^{2 s}$ and by P-initializing every node $\beta,|\beta| \equiv \bmod 5$, such that $f_{s+1}<_{L} \beta$ or there exists $\alpha<_{L} \beta$ and some $x$ such that $\widehat{\psi}_{\alpha, s+1}(x) \downarrow$ and $\widehat{\psi}_{\alpha, s}(x) \uparrow$. P-initializing $\beta$ means canceling any $\beta$-witness, and defining $\Gamma_{s+1}^{\beta}=\max \left\{\Gamma_{s}^{\beta}, s+1\right\}$.

7.6. Step 6. (Defining $m(\alpha, s+1), \mathcal{L}_{s+1}$ and $\left.Y_{\lambda, s+1}\right)$ This step applies to all four copies of the tree. It should be performed only if none of Steps 1-5 ( $\widehat{1}-\widehat{5})$ applied at this stage.

Substep 6A. (Defining $m(\alpha, s+1), \mathcal{L}_{s+1}$, and their duals) For every $\alpha \preceq f_{s+1}$ if every $\alpha$-entry $\langle\alpha, \nu\rangle$ on $\mathcal{L}_{s}$ and every $\alpha$-entry $\langle\alpha, \widehat{\nu}\rangle$ on $\widehat{\mathcal{L}}_{s}$ is marked we say that the lists are $\alpha$-marked and we

(6.1) define $m(\alpha, s+1)=m(\alpha, s)+1$, and

(6.2) add to the bottom of list $\mathcal{L}_{s}\left(\widehat{\mathcal{L}}_{s}\right)$ a new (unmarked) $\alpha$-entry $\langle\alpha, \nu\rangle(\langle\alpha, \widehat{\nu}\rangle)$ for every such $\alpha \neq \lambda$ and every $\nu \in \mathcal{M}_{\alpha}$. Let the resulting list be $\mathcal{L}_{s+1}\left(\widehat{\mathcal{L}}_{s+1}\right)$.

If the lists are not both $\alpha$-marked, then let $m(\alpha, s+1)=m(\alpha, s), \mathcal{L}_{s+1}=\mathcal{L}_{s}$ and $\widehat{\mathcal{L}}_{s+1}=\widehat{\mathcal{L}}_{s}$.

Substep 6B. (Emptying $R_{\alpha}$ to the right of $f_{s+1}$ ) For every $\alpha$ such that $f_{s+1}<_{L} \alpha$, initialize $\alpha$.

Substep 6C. (Filling $Y_{\lambda}$ and $\left.\widehat{Y}_{\lambda}\right)$ Choose the least $x \notin Y_{\lambda, s}\left(\widehat{x} \notin \widehat{Y}_{\lambda, s}\right)$ and $x<s$. Put $x$ in $S_{\lambda}^{\bar{A}}\left(\widehat{x}\right.$ in $\left.\widehat{S}_{\lambda}^{\bar{B}}\right)$.

For each $x \in Y_{\lambda, s+1}\left(\widehat{x} \in \widehat{Y}_{\lambda, s+1}\right)$ let $\alpha(x, s+1)(\alpha(\widehat{x}, s+1))$ denote the unique $\gamma$ such that $x \in S_{\gamma, s+1}\left(\widehat{x} \in \widehat{S}_{\gamma, s+1}\right)$.

(Note that after each application of Step 6 , the other Steps $1-5$ and Steps $\widehat{1}-\widehat{5}$ can apply only finitely often until the next application of Step 6 as we prove in Lemma 8.7.) 


\section{THE VERIFICATION}

We now verify that this construction establishes the theorem. Many of the lemmas in this section are similar to lemmas in [4. The most significant and important new or altered lemmas are Lemma 8.8 and Lemma 8.9, which contain the key elements of combining the automorphism construction with the construction of a nonlow set. Lemma 8.12 also has important changes.

First define the true path $f$ of the construction by induction on $e$. Let $f\lceil 0=\lambda$, the empty node. Given $\beta=f\lceil 5 e$ define $f(5 e)=0$ if $\beta$ appoints infinitely many $\beta$-witnesses and $f(5 e)=p+1$ if $p \beta$-witnesses are ever enumerated into $D$. For $\alpha=f \uparrow n$, where $n \neq 5 e$, define $f(n)$ as in the automorphism construction in Harrington-Soare [4].

Lemma 8.1. At stage $s+1$,

(i) if $x$ enters $R_{\alpha}, \alpha \neq \lambda$, then Step 1 or Step 2 applies to $\alpha$ and $x$. (This does not hold for $R_{\alpha}^{A}$.)

(ii) if $x$ moves from $S_{\alpha}$ to $S_{\delta}$, then one of the following steps must apply to $x$ : Step $1_{\delta}$ for $\delta<_{L} \alpha$ or $\delta^{-}=\alpha$; Step $2_{\delta}$ for $\delta$ such that $\delta^{-}=\alpha$; or Step $6_{\alpha}$ Substep $B$ applying to $\alpha$, so $f_{s+1}<_{L} \alpha$;

(iii) if $x \in S_{\alpha, s}$ is enumerated in a red set $U_{\alpha}$ at stage $s+1$, then Step 1 or Step 4 must apply to $x$;

(iv) if $x \in S_{\alpha, s}$ is enumerated in a blue set $\widehat{V}_{\alpha}$, then Step 1, Step 3, or Step 5 must apply to $x$.

Proof. This follows directly from the construction.

Lemma 8.2 (True Path Lemma). $f=\liminf _{s} f_{s}$.

Proof. Since $f$ is $\Pi_{2}^{0}$, we know that such a computable approximation is possible. The definition of $f_{s}$ in the $D$-Module has been formulated precisely so that $f=$ $\liminf _{s} f_{s}$, where $f$ is as in Definition 6.3.

Lemma 8.3. $\mathcal{M}_{\rho}=\mathcal{F}_{\lambda}^{+}$and $k_{\rho}=k_{\lambda}^{+}$.

Proof. We know that $A$ is infinite and co-infinite, so there are infinitely many elements $x$ such that $\nu^{+}(\lambda, x, s)$ will contain $U_{0}$ and infinitely many that will not, and will appear to $D$ not to contain $U_{0}$. Thus, $\mathcal{F}_{\lambda}^{+}$contains both possible states. Because of this, it is easy to see that $k_{\lambda}^{+}$must be 0 , as it is not possible for any element to be in a state that is not well-visited, since both states are well-visited.

8.1. The lemmas of motion, $Y_{\alpha}$, and $\alpha(x, s)$. We now verify the properties we stated in the three subsections $\$ 5.2, \$ 5.3$, and $\$ 5.4$. For each lemma there are obvious dual lemmas with similar proofs unless we state and prove the dual explicitly.

Lemma 8.4. For all $\alpha \in T$,

(i) $f<_{L} \alpha \Longrightarrow R_{\alpha, \infty}=\emptyset$,

(ii) $\alpha<_{L} f \Longrightarrow Y_{\alpha}={ }^{*} \emptyset$,

(iii) $\alpha \prec f \Longrightarrow Y_{<\alpha}:=\bigcup\left\{Y_{\delta}: \delta<_{L} \alpha\right\}={ }^{*} \emptyset$.

Proof. (i) Given $x$ choose $s>x$ such that $f_{s}<_{L} \alpha$. By Step $6 \mathrm{~B}, R_{\alpha, s}=\emptyset$. Now $x$ is $\gamma$-ineligible for all $t \geq s$ and all $\gamma \succeq \alpha$, so $x \notin S_{\gamma, t}$ and hence $x \notin R_{\alpha, t}$ by (1.3) and (2.3). 
(ii) Assume $\alpha<_{L} f$. Then $\alpha \prec f_{s}$ for finitely many $s$ and there are only finitely many $\alpha$-entries $\langle\alpha, \nu\rangle$ on the list $\mathcal{L}$ under (6.2). Hence, finitely many $x$ enter $S_{\alpha}$ under Step 1 because every such $x$ must mark some unmarked $\alpha$-entry on $\mathcal{L}$. Thus, $m(\alpha):=\lim _{s} m(\alpha, s)<\infty$ since $\mathcal{L}$ will be $\alpha$-marked at most finitely often. Hence, by (2.4), Step 2 moves only finitely many $x$ into $R_{\alpha}$. But each $x$ enters $R_{\alpha}$ only under Step 1 or Step 2, so $Y_{\alpha}={ }^{*} \emptyset$.

(iii) Immediate by (ii) since $f_{s}<_{L} \alpha$ finitely often, so there are only finitely many $\beta<_{L} \alpha$ such that $Y_{\beta} \neq \emptyset$.

Lemma 8.5. For every $\alpha \in T$ if $\alpha \neq \lambda$ and $\beta=\alpha^{-}$, then

(i) $Y_{\alpha} \backslash Y_{\beta}=\emptyset$ and $Y_{\alpha} \subseteq Y_{\beta}$ (however, elements may enter $Y_{\alpha}^{A}$ at the same time as they enter $\left.Y_{\beta}^{A}\right)$,

(ii) $(\forall x)(\exists \leq 1 s)\left[x \in R_{\alpha, s+1}-R_{\alpha, s}\right]$,

(iii) $U_{\alpha} \backslash Y_{\alpha}=\widehat{V}_{\alpha} \backslash Y_{\alpha}=\emptyset$, except that elements may enter $A=U_{\rho}\left(B=\widehat{U}_{\rho}\right)$ before entering $Y_{\rho}\left(\widehat{Y}_{\rho}\right)$.

(iv) If $\alpha \prec f$, then

$$
\left(\exists v_{\alpha}\right)(\forall x)\left(\forall s \geq v_{\alpha}\right)\left[\begin{array}{c}
x \in R_{\alpha, s}^{\bar{A}} \Longrightarrow(\forall t \geq s)\left[x \in R_{\alpha, t}^{\bar{A}} \text { or } x \in R_{\alpha, t}^{A}\right] \\
\text { and } x \in R_{\alpha, s}^{A} \Longrightarrow(\forall t \geq s)\left[x \in R_{\alpha, t}^{A}\right]
\end{array}\right] .
$$

Proof. (i) Suppose $x \in Y_{\alpha, s+1}-Y_{\alpha, s}$. Then at stage $s+1$ either Step 1, Step 2, or switching applies to $x$ and $\alpha$, so $x \in Y_{\beta, s}$ by (1.1) and (2.1), unless switching occurred, in which case $x \in Y_{\beta, s+1}^{A}$.

(ii) Suppose $x \in R_{\alpha, s+1}-R_{\alpha, s}$ and $x \in R_{\alpha, t}-R_{\alpha, t+1}$ for some $t>s$. Then $x<s$ by Step 6C. Hence, by Lemma 8.1(ii) at stage $t+1$ either: (1) Step 6B applies to $\alpha$ and $x$, or (2) Step 1 applies to $\delta$ and $x$ for some $\delta<_{L} \alpha, \delta=\alpha(x, t+1)$. If (1), then $f_{s+1}<_{L} \alpha$, so $x$ is $\gamma$-ineligible at all stages $v \geq t+1$ and all $\gamma \succeq \alpha$, and $x$ can never reenter $R_{\alpha}$ because of (1.3) and (2.3). If (2), then by Lemma 8.1(ii), (1.4), and induction on $v \geq t$, either for all $v \geq t, \alpha(x, v)<_{L} \alpha$ so $x \notin R_{\alpha, v}$, or else Step 6B applies at stage $v+1$ to $x$ and some $\eta<_{L} \alpha, \eta=\alpha(x, v)$, in which case the argument for (1) shows that $x \notin R_{\alpha, w}$, for all $w \geq v$.

(iii) Enumeration of $x$ in $U_{\alpha, s+1}\left(\widehat{V}_{\alpha, s+1}\right)$ takes place only under Step 1, in which case $x \in Y_{\alpha, s+1}$, or under Step 4 (respectively, Step 3 or Step 5), in which case $x \in Y_{\alpha, s}$ already. However, $x$ may enter $U_{\rho, s+1}$ directly from $S_{\lambda}$ by Step 1 .

(iv) Assume $\alpha \prec f$. Choose $v_{\alpha}$ such that for $s \geq v_{\alpha}, f_{s} \nless_{L} \alpha$, and no $\beta<_{L} \alpha$ acts at stage $s$, and hence $Y_{<\alpha, s}=Y_{<\alpha}$. Thus, if $x \in R_{\alpha, s}$ for $s \geq v_{\alpha}$, then $x$ cannot be pulled to $S_{\gamma}$ for $\gamma<_{L} \alpha$ by Step $1_{\gamma}$ and $x$ cannot be removed from $R_{\alpha}$ by Step $6 \mathrm{~B}$, so $x$ must remain in $R_{\gamma, t}$ for all $t \geq s$ or else be enumerated into $A$ if it has not already been.

Lemma 8.6. For all $x \in \omega$,

(i) $\alpha(x):=\lim _{s} \alpha(x, s)$ exists, and

(ii) $x$ is enumerated in at most finitely many c.e. sets $U_{\gamma}, \widehat{V}_{\gamma}$, and hence for $\alpha=\alpha(x)$,

$$
\nu(\alpha, x):=\lim _{s} \nu(\alpha, x, s) \text { exists. }
$$

Proof. (i) By (1.2), (2.2), and Lemma 8.1(i), $x \in S_{\alpha, s}$ implies $x>|\alpha|$ and $x>p$ if $\alpha=\beta^{\wedge} p$ and $|\alpha|=5 e$. Fix $x$, let $\gamma=f\left\lceil x\right.$ and choose $s>v_{\gamma}$ (as defined in Lemma 8.5(iv)) such that $\gamma \prec f_{s}$. Let $\delta_{0}=\alpha(x, s)$. Clearly, $\delta_{0}<_{L} \gamma$ or $\delta_{0} \preceq \gamma$ by 
Step 6B. Also by induction on $t \geq s$, if $\delta_{k}=\alpha(x, t)$ and $\delta_{k+1}=\alpha(x, t+1)$, then $\delta_{k+1}<_{L} \delta_{k}$ or $\delta_{k+1} \succ \delta_{k}$ because Step 1 or Step 2 must have applied to $\delta_{k}$ and $x$ at stage $t+1$ since Step $6 \mathrm{~B}$ cannot apply to $x$ after stage $v_{\gamma}$. But there is no infinite sequence $\left\{\delta_{0}, \delta_{1}, \ldots\right\}$ such that for all $k, \delta_{k+1}<_{L} \delta_{k}$ or $\delta_{k+1} \succ \delta_{k}$, where $x$ still satisfies (1.2) and (2.2).

(ii) By (i) choose $t_{x} \geq v_{\gamma}$ such that $\alpha(x, s)=\alpha$ for all $s \geq t_{x}$. Then $\nu(\alpha, x, s) \subseteq$ $\nu(\alpha, x, s+1)$ for all $s \geq t_{x}$. Hence,

$$
\nu(\alpha, x)=\bigcup\left\{\nu(\alpha, x, s+1): s \geq t_{x}\right\},
$$

where this union is defined as in Definition 5.6(iv).

Lemma 8.7. (i) Step 6 applies infinitely often.

(ii) If the hypotheses of some Steps $1-5$ (Steps $\widehat{1}-\widehat{5})$ remain satisfied, then that step eventually applies.

Proof. (i) If Step 6 applies at stage $s$, then the finitely many $x \in Y_{\lambda, s}\left(\widehat{x} \in \widehat{Y}_{\lambda, s}\right)$ remain the same until the next application of Step 6. Each later application of Steps $1-5$ (Steps $\widehat{1}-\widehat{5})$ chooses some $x(\widehat{x})$ to change position or to be enumerated in some set $U_{\gamma}$ or $\widehat{V}_{\gamma}\left(\widehat{U}_{\gamma}\right.$ or $\left.V_{\gamma}\right)$. By Lemma 8.6 this can happen at most finitely often for each $x \in Y_{\lambda, s}\left(\widehat{x} \in \widehat{Y}_{\lambda, s}\right)$. Hence, Step 6 applies at some stage $t>s$.

(ii) Step 6 cannot apply at stage $t$ if the hypotheses for some Steps 1-5 (Steps $\widehat{1}-\widehat{5})$ are satisfied because the latter steps are performed before Step 6 by the basic construction in $₫ 7$

8.2. Showing that $\mathcal{M}_{\alpha} \subseteq \mathcal{E}_{\alpha}$. The following lemma is based on Lemma 3.3. While the statement may seem entirely different, both lemmas show the satisfaction of negative requirements, which are interacting with positive requirements in the same way.

Lemma 8.8. For all $\alpha \prec f$ and all $\nu_{e} \in \mathcal{M}_{\alpha}^{\bar{A}}$,

$$
\left(\exists^{\infty} s\right)\left[y_{\langle e, k\rangle}^{s} \text { exists }\right] \quad \Longrightarrow \quad \lim _{s} y_{\langle e, k\rangle}^{s} \text { exists and is in } \bar{A} \text {. }
$$

Proof. Induct on the length of $\alpha$ and on $k$. Let $\nu_{e} \in \mathcal{M}_{\alpha}^{\bar{A}}$. Assume true for all $\beta \prec \alpha$ and all $\langle e, i\rangle$ with $i<k$.

Let $s_{0}$ be such that $y_{\langle e, i\rangle}^{s} \in \bar{A}$ for all $s \geq s_{0}$ and all $i<k$.

Consider all the finitely many pairs $\langle\beta, n\rangle\langle\langle\alpha, e, k\rangle$. Let $p$ be the maximum of $|\beta|$ for such a $\beta$. Choose some $\xi$ associated with the positive requirement of making $D$ nonlow (i.e. $|\xi| \equiv 4 \bmod 5$ ), such that $|\xi|>p$, and $\alpha \prec \xi \prec 0 \prec f$. Choose $s_{1} \geq s_{0}$ such that for all $s \geq s_{1}$ : (a) if $\langle\beta, n\rangle\left\langle\langle\alpha, e, k\rangle\right.$, then the $\beta$-witness $x_{n}^{\beta}$ does not become newly defined or enter $D$ at stage $s$; (b) $f_{s} \nless_{L} \xi$ (so every $\beta<_{L} \xi$ with $|\beta| \equiv 4 \bmod 5$ has ceased to act by stage $s$ ); and (c) every $\beta$ with $\beta^{\prec p} \prec \xi$ for $p \neq 0$ has ceased to act by stage $s$. By choice of $s_{1}$ no witness $x_{n}^{\beta}$, for $\beta<_{L} \xi$ or $\beta \prec p \prec \xi$ for $p \neq 0$, will ever contribute a $\beta$-witness to $D$ after stage $s_{1}$.

Choose $s_{2} \geq s_{1}$ such that $\xi$ contributes a $\xi$-witness $x_{m}^{\xi}$ to $D$ at $s_{2}$. This action of $\xi$ causes P-initialization of all $\beta$ with $\xi<_{L} \beta$, and cancellation of all such $\beta$-witnesses at stage $s_{2}$. By (73), all $\beta, \beta^{\prec} 0 \prec \xi$, must have contributed their witnesses to $D$ at stage $s_{2}$, and so have no $\beta$-witnesses in existence at the end of stage $s_{2}$.

Choose $s_{3} \geq s_{2}$ such that $y_{\langle e, k\rangle}^{s_{3}}$ is defined. 
Consider $\xi \prec \beta$. Suppose $\langle\beta, n\rangle>\langle\alpha, e, k\rangle$ and $x_{n}^{\beta}$ is a witness that exists at stage $s_{3}$. If $x_{n}^{\beta}$ was appointed when $\widehat{\psi}_{\alpha, t}\left(y_{\langle e, k\rangle}^{t}\right) \geq \widehat{\psi}_{\alpha, s_{3}}\left(y_{\langle e, k\rangle}^{s_{3}}\right)$, then $x_{n}^{\beta}$ must be larger than $\widehat{\psi}_{\alpha, s_{3}}\left(y_{\langle e, k\rangle}^{s_{3}}\right)$, so it will not injure it. However, if $x_{n}^{\beta}$ was appointed when $\widehat{\psi}_{\alpha, t}\left(y_{\langle e, k\rangle}^{t}\right)<\widehat{\psi}_{\alpha, s_{3}}\left(y_{\langle e, k\rangle}^{s_{3}}\right)$, then at some stage after $s_{3}, x_{n}^{\beta}$ could injure the current computation. Now, the only way for this to happen is for something else to injure the computation before stage $s_{3}$ and after $x_{n}^{\beta}$ has been appointed. The injury could not come from a node $\gamma<_{L} \beta$ because that would reset the $\beta$-witness. It could not come from a node $\gamma \succ \beta$ because by (73), $\gamma$ must wait for $\beta$ to enumerate its witness before enumerating its own. Suppose the injury came from a node $\gamma$ to the right of $\beta$. Then to avoid the $\gamma$-witness being reset, $x_{n}^{\beta}$ must be defined before the $\gamma$-witness. Thus, by (72), $\gamma$ must have its witness larger than $x_{n}^{\beta}$, which means that $\gamma$ could not have injured the $\langle\alpha, y\rangle$ computation. Now, if $\gamma \widehat{\gamma} p \prec \beta$ for any $p \neq 0$, then by (71), $\beta$ must wait for all $\gamma$-witnesses to enter $D$ before defining its own witness. If $\gamma^{\wedge} 0 \prec \beta$, then by (72), $\beta$ must choose its witness smaller than the $\gamma$-witness, so $\gamma$ cannot injure the $\langle\alpha, y\rangle$ computation. Thus, no $\beta$-witness for $\xi \prec \beta$ appointed before stage $s_{1}$ can possibly injure $\widehat{\psi}_{\alpha, s_{3}}\left(y_{\langle e, k\rangle}^{s_{3}}\right)$.

By the time $y_{\langle e, k\rangle}^{s_{3}}$ is defined, the only $\beta$-witnesses in existence which have the priority to injure a computation $\widehat{\psi}_{\alpha, t}(x)$ for some $t \geq s_{3}$ are those which are permanently dormant and will never enter $D$.

Consider any new $\beta$-witness $x_{n}^{\beta}$ appointed at or after stage $s_{3}$. We cannot have $\beta<_{L} \xi$ by (b), or $\beta^{\prec} p \prec \xi$ by (c). If $\alpha \preceq \beta$, then we must have $\widehat{\psi}_{\alpha, s}\left(y_{\langle e, k\rangle}^{s}\right) \downarrow<x_{n}^{\beta}$ by (72). If $f<_{L} \beta$, then $x_{n}^{\beta}$ must exceed $\widehat{\psi}_{\alpha, s}\left(y_{\langle e, k\rangle}^{s}\right)$ if the latter converges when $x_{n}^{\beta}$ is appointed, and if not, $x_{n}^{\beta}$ must be canceled whenever it later converges. (Note that any $x_{n}^{\beta}$ newly appointed at $s$ must exceed $\widehat{\psi}_{\alpha}^{D}\left(y_{\langle e, k\rangle}^{s}\right)[s]$ because the former must exceed $s$ by the definition of $\Gamma_{s}^{\beta}$, and the latter must be less than $s$ by the usual convention on the use function.) $A$.

Hence, $\widehat{\psi}_{\alpha, s_{3}}\left(y_{\langle e, k\rangle}^{s_{3}}\right)$ is never injured, so $y_{\langle e, k\rangle}^{s_{3}}=\lim _{s} y_{\langle e, k\rangle}^{s}$ and does not enter

Lemma 8.9. Let $\alpha \prec f$. Then

(i) $\left(\forall \gamma<{ }_{L} f\right)\left[m(\gamma):=\lim _{s} m(\gamma, s)<\infty\right]$,

(ii) $m(\alpha):=\lim _{s} m(\alpha, s)=\infty$,

(iii) $\left(\forall \nu_{e} \in \mathcal{M}_{\alpha}^{\bar{A}}\right)(\forall k)\left(\exists^{\infty} s\right)\left[y_{\langle e, k\rangle}^{s}\right.$ exists $]$,

(iv) $\left(\forall \nu_{e} \in \mathcal{M}_{\alpha}^{\bar{A}}\right)(\forall k)\left[\lim _{s} y_{\langle e, k\rangle}^{s}\right.$ exists and is not in $\left.A\right]$,

(v) $\mathcal{E}_{\alpha} \supseteq \mathcal{M}_{\alpha}$, and

(vi) $\widehat{\mathcal{E}}_{\alpha} \supseteq \widehat{\mathcal{M}}_{\alpha}$.

Proof. (i) If $\gamma<_{L} f$, then $\gamma \prec f_{s}$ for finitely many $s$, so finitely many $\gamma$-entries are ever added to $\mathcal{L}$ and hence $\mathcal{L}$ is $\gamma$-marked finitely often and $m(\gamma)<\infty$.

For (ii)-(vi), induct on $\alpha$.

For the base case, $\alpha=\lambda$, we have (ii) because we never put any entries on the $\lambda$-list, so every time Step 6 acts, $m(\lambda, s)$ increases. Part (iii) follows by the fact that $\bar{A}$ is infinite, and Lemma 8.8 gives (iv). For (v) and (vi), we have $\mathcal{M}_{\lambda} \subset \mathcal{E}_{\lambda}$ and its dual simply because Step 6 acts infinitely often.

Assume the theorem is true for all $\gamma \prec \alpha$.

Suppose for a contradiction that $m(\alpha)<\infty$, say $m(\alpha, s)=m_{0}$ for all $s \geq s_{0}$. 
Claim 1. Every $\alpha$-entry $\left\langle\alpha, \nu_{1}\right\rangle$ on $\mathcal{L}\left(\left\langle\alpha, \widehat{\nu}_{1}\right\rangle\right.$ on $\left.\widehat{\mathcal{L}}\right)$ is eventually marked.

Proof.

Case 1. The first unmarked entry is in $\mathcal{M}_{\alpha}^{A}$.

Suppose that some $\alpha$-entry $\left\langle\alpha, \nu_{1}\right\rangle$ on $\mathcal{L}$ is never marked, where $\nu_{1} \in \mathcal{M}_{\alpha}^{A}$. Hence, by Step $6 \mathrm{~A}$ there are only finitely many $\alpha$-entries on $\mathcal{L}$. Choose $s_{1} \geq s_{0}$ such that every $\alpha$-entry on $\mathcal{L}$ and every entry on $\mathcal{L}$ preceding $\left\langle\alpha, \nu_{1}\right\rangle$ which will ever be marked is marked by stage $s_{1}, Y_{<\alpha, s_{1}}=Y_{<\alpha}$, and for all $x \leq m_{0}, x \in Y_{\alpha, s_{1}}$ iff $x \in Y_{\alpha}$.

Suppose $e_{\alpha}>e_{\beta}$. Then $\nu_{1} \in \mathcal{M}_{\alpha}=\mathcal{F}_{\beta}^{+}$since $\alpha \prec f$. Thus,

$$
\left(\exists^{\infty} x\right)\left(\exists s>s_{1}\right)\left[x \in Y_{\beta, s}^{A} \& \nu^{+}(\alpha, x, s)=\nu_{1}\right] .
$$

By the choice of $s_{1}$ almost every such $x$ also satisfies (1.1)-(1.8). Thus, some such $x$ is moved to $S_{\alpha}$ under Step 1 at some stage $s+1>s_{1}$ and the entry $\left\langle\alpha, \nu_{1}\right\rangle$ is then marked, contrary to hypothesis.

Suppose $e_{\alpha}=e_{\beta}$. Now $\nu_{1} \in \mathcal{M}_{\alpha}$, so $\nu_{1} \uparrow \beta \in \mathcal{M}_{\beta}$. By the induction hypothesis, $\nu_{1}\left\lceil\beta \in \mathcal{E}_{\beta}\right.$. Thus

$$
\left(\exists^{\infty} x\right)\left(\exists s>s_{1}\right)\left[x \in Y_{\beta, s}^{A} \& \nu(\beta, x, s)=\nu_{1}\lceil\beta] .\right.
$$

By the choice of $s_{1}$ almost every such $x$ also satisfies (1.1)-(1.8). Thus, some such $x$ is moved to $S_{\alpha}$ under Step 1 at some stage $s+1>s_{1}$ and the entry $\left\langle\alpha, \nu_{1}\right\rangle$ is then marked, contrary to hypothesis.

Case 2. The first unmarked entry is in $\mathcal{M}_{\alpha}^{\bar{A}}$.

Suppose that some $\alpha$-entry $\left\langle\alpha, \nu_{1}\right\rangle$ on $\mathcal{L}$ is never marked, where $\nu_{1} \in \mathcal{M}_{\alpha}^{\bar{A}}$. Hence, by Step $6 \mathrm{~A}$ there are only finitely many $\alpha$-entries on $\mathcal{L}$. Choose $s_{1} \geq s_{0}$ such that every $\alpha$-entry on $\mathcal{L}$ and every entry on $\mathcal{L}$ preceding $\left\langle\alpha, \nu_{1}\right\rangle$ which will ever be marked is marked by stage $s_{1}, Y_{<\alpha, s_{1}}=Y_{<\alpha}$, and for all $x \leq m_{0}, x \in Y_{\alpha, s_{1}}$ iff $x \in Y_{\alpha}$.

Suppose $e_{\alpha}>e_{\beta}$. Then $\mathcal{M}_{\alpha}=\mathcal{F}_{\beta}^{+}$since $\alpha \prec f$. So

$$
\left(\exists^{\infty} x\right)\left(\exists s>s_{1}\right)\left[x \in Y_{\beta, s}, \nu^{+}(\alpha, x, s)=\nu_{1}, \widehat{\Psi}_{\alpha}^{D}(x)[s] \downarrow=0\right] .
$$

By the choice of $s_{1}$ almost every such $x$ also satisfies (1.1)-(1.7) of Step 1. Thus, some such $x$ is moved to $S_{\alpha}$ under Step 1 at some stage $s+1>s_{1}$ and the entry $\left\langle\alpha, \nu_{1}\right\rangle$ is then marked, contrary to hypothesis.

Suppose $e_{\alpha}=e_{\beta}$. Now $\nu_{1} \in \mathcal{M}_{\alpha}$, so $\nu_{1}\left\lceil\beta \in \mathcal{M}_{\beta}\right.$. By the induction hypothesis,

$$
(\forall k)\left(\forall^{\infty} s\right)\left[\exists x=y_{\left\langle e^{\prime}, k\right\rangle}^{s} \text { where } x \notin A\right],
$$

where $\nu_{e^{\prime}}=\nu_{1}\left\lceil\beta\right.$. By the choice of $s_{1}$, almost all of these must stay in $S_{\beta}$. Since $x$ is not allowed to change states without appearing to not be in $A$, these $x$ values must at some stage be in $S_{\beta}$, appear to be in $\bar{A}$, and be in state $\nu_{1} \uparrow \beta$. By the choice of $s_{1}$ almost every such $x$ also satisfies (1.1)-(1.7). Thus, some $x$ is moved to $S_{\alpha}$ under Step 1 at some stage $s+1>s_{1}$ and the entry $\left\langle\alpha, \nu_{1}\right\rangle$ is then marked, contrary to hypothesis. This proves the claim.

To complete the proof of (ii) use the claim to find $s>s_{0}$ such that $\alpha \prec f_{s+1}$ and every $\alpha$-entry on $\mathcal{L}_{s}$ and $\widehat{\mathcal{L}}_{s}$ is marked. Now by Step $6 \mathrm{~A}, m(\alpha, s+1)>m(\alpha, s)=m_{0}$, contrary to the choice of $s_{0}$.

For (iii), induct on $k$. Suppose it is true for all $i<k$. Then by Theorem 8.8, if $k>0$, then $\lim _{s} y_{\langle e, k-1\rangle}^{s}=y_{\langle e, k-1\rangle}$ exists and is not in $A$. Since $m(\alpha)=\infty$ by part (ii), we know that infinitely many elements are pulled up into $\nu_{e}$ by Step 1. Thus, 
$y_{\langle e, k\rangle}^{s}$ will be infinitely often defined. (Note that $y_{\langle e, k\rangle}^{s}$ may be defined during stage $s$ before any elements enter $D$ in stage $s$.) Part (iv) follows by Theorem 8.8

Parts (v) and (vi) follow by (ii), since every element of $\mathcal{M}_{\alpha}$ is marked by Step 1.

\subsection{Completing the automorphism verification.}

Lemma 8.10. $\alpha \prec f \Longrightarrow R_{\alpha, \infty}={ }^{*} Y_{\alpha}={ }^{*} Y_{\lambda}=\omega$.

Proof. By Lemma 8.7(i), Step 6C must eventually put every element $x \in \omega$ into $Y_{\lambda}$. By induction we may assume $R_{\beta, \infty}={ }^{*} Y_{\beta}={ }^{*} \omega$, for $\beta^{-}=\alpha$. By Lemma 8.9, $m(\alpha)=\infty$ and $m(\gamma)<\infty$ for all $\gamma<_{L} \alpha$ with $\gamma^{-}=\beta$.

By Lemma 8.4, $Y_{<\alpha}={ }^{*} \emptyset$ and almost every $x \in R_{\beta}$ not yet in $R_{\alpha}$ must eventually lie in $S_{\beta}$. Hence, almost every $x \in R_{\beta}^{\bar{A}}$ not yet in $R_{\alpha}^{\bar{A}}$ must eventually satisfy the conditions of Step $2^{\bar{A}}$ and must eventually move to $S_{\alpha}$ by Step 2, or else be enumerated into $A$. Once in $A$, almost every $x \in R_{\beta}^{A}$ not yet in $R_{\alpha}^{A}$ must eventually satisfy the conditions of Step $2^{A}$ and be moved to $S_{\alpha}$ by Step 2. By Lemma 8.5)(iv) almost every such $x \in \bar{A}$ will remain in $R_{\alpha}$ forever.

Lemma 8.11. $\alpha \prec f \Longrightarrow \alpha$ is $\mathcal{M}$-consistent.

Proof. Let $\alpha \prec f$ and $\beta=\alpha^{-}$. Assume for a contradiction that $\alpha$ is not $\mathcal{M}$ consistent. Then $e_{\alpha}>e_{\beta}$ and there exist $\nu_{0} \in \mathcal{M}_{\alpha}, \nu_{1} \notin \mathcal{M}_{\alpha}, \nu_{0}<_{B} \nu_{1}$ and $\nu_{1} \uparrow \beta \in \mathcal{M}_{\beta}$.

By (66), $\alpha$ is a terminal node on $T$, so $S_{\alpha}=R_{\alpha}$. By Lemmas 8.10 and 8.5 (iv), $S_{\alpha, \infty}{ }^{*} \omega$ and no $x \in S_{\alpha, s}, s>v_{\alpha}$, later leaves $S_{\alpha}$. By Lemma 8.9, $\mathcal{E}_{\alpha} \supseteq \mathcal{M}_{\alpha}$, and

$\left(\exists^{\infty} x\right)(\exists s)\left[x \in S_{\alpha, s+1}-S_{\alpha, s}\right.$ by Step 1, and after Step 1, $x$ is in state $\left.\nu_{0}\right]$.

Choose any such $x$ and $s>v_{\alpha}$. At the end of Step 1, Step 3 will act and enumerate $x$ into such sets that $\nu(\alpha, x, s+1)=\nu_{1}$. Thus, $\alpha$ is provably incorrect at all stages $v>s+1$, so $\alpha \nprec f$.

Lemma 8.12. If $\alpha \prec f$, then

(i) $\widehat{\mathcal{M}}_{\alpha}=\left\{\widehat{\nu}: \nu \in \mathcal{M}_{\alpha}\right\}$,
(ii) $\mathcal{M}_{\alpha}=\mathcal{F}_{\alpha}=\mathcal{E}_{\alpha}$, and
(iii) $\widehat{\mathcal{M}}_{\alpha}=\widehat{\mathcal{F}}_{\alpha}=\widehat{\mathcal{E}}_{\alpha}$.

Proof. Fix $\alpha \prec f$, and let $\beta=\alpha^{-}$. Now (i) holds by the definitions of $\mathcal{M}_{\alpha}$ and $\widehat{\mathcal{M}}_{\alpha}$. Assume (ii) and (iii) for $\beta$. We know $\mathcal{E}_{\alpha} \subseteq \mathcal{F}_{\alpha}$ by their definitions, and $\mathcal{M}_{\alpha} \subseteq \mathcal{E}_{\alpha}$ by Lemma 8.9. Thus, to prove (ii) (and (iii)) it suffices to prove $\mathcal{F}_{\alpha} \subseteq \mathcal{M}_{\alpha}$ (and $\widehat{\mathcal{F}}_{\alpha} \subseteq \widehat{\mathcal{M}}_{\alpha}$ ). Induct on $\alpha$. Suppose the theorem holds for all $\beta \prec \alpha$.

Case 1. $e_{\alpha}=e_{\beta}$ and $\widehat{e}_{\alpha}=\widehat{e}_{\beta}$.

Then $\mathcal{M}_{\alpha}=\mathcal{M}_{\beta}$. Also $\mathcal{F}_{\alpha} \subseteq \mathcal{F}_{\beta}$ since $Y_{\alpha} \subseteq Y_{\beta}$. Finally, $\mathcal{M}_{\beta}=\mathcal{F}_{\beta}$ by the inductive hypothesis (ii) for $\beta$. Hence,

$$
\mathcal{F}_{\alpha} \subseteq \mathcal{F}_{\beta}=\mathcal{M}_{\beta}=\mathcal{M}_{\alpha},
$$

so (ii) holds for $\alpha$. Likewise, $\widehat{\mathcal{F}}_{\alpha} \subseteq \widehat{\mathcal{M}}_{\alpha}$, so (iii) holds for $\alpha$.

Before considering Cases 2 and 3 we need a technical sublemma. 
Sublemma. If $e_{\alpha}>e_{\beta}, \nu_{2}=\left\langle\alpha, \sigma_{2}, \tau_{2}\right\rangle \in \mathcal{F}_{\beta}^{+}$, and $\nu_{1}=\left\langle\alpha, \sigma_{1}, \tau_{2}\right\rangle$, where $\sigma_{1}=$ $\sigma_{2}-\left\{e_{\alpha}\right\}$, then $\nu_{1} \in \mathcal{F}_{\beta}^{+}$also.

Proof. Suppose $\nu_{2} \in \mathcal{F}_{\beta}^{+}$. Then $\nu_{3}=\nu_{2} \uparrow \beta \in \mathcal{M}_{\beta}$.

Suppose $\nu_{3} \in \mathcal{M}_{\beta}^{\bar{A}}$. By Lemma 8.9 , for every $k$, there is an $x$ that enters $\nu_{3}$ by Step 1, and immediately becomes the final $y_{\langle e, k\rangle}^{s}$ for the appropriate $e$. Hence,

$$
\left(\exists^{\infty} x\right)(\exists s)\left[x \in Y_{\beta, s}-Y_{\beta, s-1}, \nu(\beta, x, s)=\nu_{3}, \widehat{\Psi}_{\beta}^{D}(x)[s] \downarrow=0\right] .
$$

However, for each such $x$ and $s, x \notin Z_{e_{\alpha}, s}$ (by the definition of $Z_{e_{\alpha}, s}$ in 96.1 ) so $\nu^{+}(\alpha, x, s)=\nu_{1}$. Hence, $\nu_{1} \in \mathcal{F}_{\beta}^{+}$by the definition of $\mathcal{F}_{\beta}^{+}$because $\widehat{\psi}_{\beta, s}(x)=\widehat{\psi}_{\alpha, s}(x)$ since $\beta$ is not a positive requirement node.

Suppose $\nu_{3} \in \mathcal{M}_{\beta}^{A}$. Then $\nu_{3} \in \mathcal{F}_{\beta}^{A}=\mathcal{E}_{\beta}^{A}$ by the inductive hypothesis for $\beta$. Hence, by the definition of $\mathcal{E}_{\beta}^{A}$,

$$
\left(\exists^{\infty} x\right)(\exists s)\left[x \in Y_{\beta, s}^{A}-Y_{\beta, s-1} \quad \& \nu(\beta, x, s)=\nu_{3}\right] .
$$

But for each such $x$ and $s, x \notin Z_{e_{\alpha}, s}$ (by the definition of $Z_{e_{\alpha}, s}$ in 6 6.1) so $\nu^{+}(\alpha, x, s)=\nu_{1}$. Hence, $\nu_{1} \in \mathcal{F}_{\beta}^{+}$by the definition of $\mathcal{F}_{\beta}^{+}$.

The dual of the Sublemma is proved as in the $\mathcal{M}_{\beta}^{A}$ case above, with $B$ or $\bar{B}$ in place of $A$.

Case 2. $e_{\alpha}>e_{\beta}$.

We prove $\mathcal{F}_{\alpha} \subseteq \mathcal{M}_{\alpha}$ and its dual $\widehat{\mathcal{F}}_{\alpha} \subseteq \widehat{\mathcal{M}}_{\alpha}$ in the following claims. (The proof of Case $3, \widehat{e}_{\alpha}>\widehat{e}_{\beta}$, is similar, but slightly simpler. In particular, the proof of $\widehat{\mathcal{F}}_{\alpha} \subseteq \widehat{\mathcal{M}}_{\alpha}$ is dual to Claim 1 for the $A$-side of the tree, and it is not necessary to differentiate the $B$ and $\bar{B}$ sides in the proof. The proof of $\mathcal{F}_{\alpha} \subseteq \mathcal{M}_{\alpha}$ is dual to Claims 2a, 2b, and 2c.)

Claim 1. $\mathcal{F}_{\alpha} \subseteq \mathcal{M}_{\alpha}$.

Proof. Suppose $\nu_{1} \in \mathcal{F}_{\alpha}$. Let $\nu_{1}=\left\langle\alpha, \sigma_{1}, \tau_{1}\right\rangle$. Then

$$
\left(\exists^{\infty} x\right)(\exists s)\left[x \in Y_{\alpha, s} \quad \& \quad \nu(\alpha, x, s)=\nu_{1}\right] .
$$

Note that $Y_{\alpha, s} \subseteq Y_{\beta, s}$ and $\nu(\alpha, x, s) \leq_{\mathrm{R}} \nu^{+}(\alpha, x, s)$ because $U_{\alpha, s} \subseteq Z_{e_{\alpha}, s}$.

Subcase 1. $\nu_{1} \in \mathcal{F}_{\alpha}^{\bar{A}}$.

The $Y_{\alpha, s}$ in (76) can be taken to be $Y_{\alpha, s}^{\bar{A}}$. Now, we have

$$
\left(\exists^{\infty} x\right)(\exists s)\left[x \in Y_{\beta, s}^{\bar{A}} \& \nu(\beta, x, s)=\nu_{1} \uparrow \beta\right] .
$$

By the inductive hypothesis, $\nu_{1} \uparrow \beta \in \mathcal{F}_{\beta} \subset \mathcal{M}_{\beta}$. As in Lemma 8.9 (iv), infinitely many of these $x$ values stay in $\bar{A}$ forever, and appear to be in $\bar{A}$ from the moment they enter $S_{\beta}^{\bar{A}}$ in state $\nu_{1} \uparrow \beta$. For each such $x$ that enters $S_{\beta}^{\bar{A}}$, at $s$, either $\nu^{+}(\alpha, x, s)=\nu_{1}$, in which case $\nu_{1} \in \mathcal{F}_{\beta}^{+}$since $x$ appears to be in $\bar{A}$, or $\nu^{+}(\alpha, x, s)=\nu_{2}$, where $\nu_{2}=\left\langle\alpha, \sigma_{2}, \tau_{1}\right\rangle$ and where $e_{\alpha} \notin \sigma_{1}$ and $\sigma_{2}=\sigma_{1} \cup\left\{e_{\alpha}\right\}$. Now $\nu_{2} \in \mathcal{F}_{\beta}^{+}$since $Y_{\alpha, s} \subseteq Y_{\beta, s}$, so $\nu_{1} \in \mathcal{F}_{\beta}^{+}=\mathcal{M}_{\alpha}$ by the Sublemma. 
Subcase 2. $\nu_{1} \in \mathcal{F}_{\alpha}^{A}$.

The $Y_{\alpha, s}$ in (76) can be taken to be $Y_{\alpha, s}^{A}$. First suppose

$$
\left(\exists^{\infty} x\right)(\exists s)\left[x \in Y_{\alpha, s}^{A} \& \nu^{+}(\alpha, x, s)=\nu_{1}\right]
$$

Then $\nu_{1} \in \mathcal{F}_{\beta}^{+}$by definition of $\mathcal{F}_{\beta}^{+}$because $Y_{\alpha, s}^{A} \subseteq Y_{\beta, s}^{A}$, and $\mathcal{F}_{\beta}^{+}=\mathcal{M}_{\alpha}$ since $\alpha \prec f$.

If (78) fails, then for almost every $x$ in (76) $), \nu^{+}(\alpha, x, s)=\nu_{2}>_{R} \nu_{1}$, so $\nu_{2}=$ $\left\langle\alpha, \sigma_{2}, \tau_{1}\right\rangle$, where $e_{\alpha} \notin \sigma_{1}$ and $\sigma_{2}=\sigma_{1} \cup\left\{e_{\alpha}\right\}$. Now $\nu_{2} \in \mathcal{F}_{\beta}^{+}$since $Y_{\alpha, s}^{A} \subseteq Y_{\beta, s}^{A}$, so $\nu_{1} \in \mathcal{F}_{\beta}^{+}=\mathcal{M}_{\alpha}$ by the Sublemma.

Claim 2. $\widehat{\mathcal{F}}_{\alpha} \subseteq \widehat{\mathcal{M}}_{\alpha}$.

Proof. We establish Claim 2 by the next three claims.

Claim 2a. $\widehat{\mathcal{E}}_{\alpha} \subseteq \widehat{\mathcal{M}}_{\alpha}$.

Proof. Assume $\widehat{\nu}_{1} \in \widehat{\mathcal{E}}_{\alpha}$. Hence,

$$
\left(\exists^{\infty} \widehat{x}\right)(\exists s)\left[\widehat{x} \in \widehat{S}_{\alpha, s+1}-\widehat{Y}_{\alpha, s} \& \nu(\alpha, \widehat{x}, s+1)=\widehat{\nu}_{1}\right] .
$$

For every such $\widehat{x}$ and $s, \widehat{x}$ must have entered $\widehat{S}_{\alpha, s+1}$ under Step $\widehat{1}$ or Step $\widehat{2}$. If Step $\widehat{1}$ applied, then we marked an entry $\left\langle\alpha, \widehat{\nu}_{1}\right\rangle$ on $\widehat{\mathcal{L}}_{s}$, so $\widehat{\nu}_{1} \in \widehat{\mathcal{M}}_{\alpha}$ by the definition of $\widehat{\mathcal{L}}$ in Step 6. If Step $\hat{2}$ applied, then $\widehat{x} \notin \widehat{U}_{\alpha, s+1}$ because $\widehat{x} \notin \widehat{U}_{\alpha, s}$ by Lemma 8.5 (iii) and no enumeration takes place at stage $s+1$ under Step $\widehat{2}$. Hence, $e_{\alpha} \notin \sigma_{1}$, where $\nu_{1}=\left\langle\alpha, \sigma_{1}, \tau_{1}\right\rangle$.

Let $\nu_{3}=\nu_{1} \uparrow \beta$. Now $\widehat{\nu}_{3} \in \widehat{\mathcal{F}}_{\beta}=\widehat{\mathcal{M}}_{\beta}$, so $\nu_{3} \in \mathcal{M}_{\beta}=\mathcal{F}_{\beta}$ and thus either $\nu_{1} \in \mathcal{F}_{\beta}^{+}$ or $\nu_{2} \in \mathcal{F}_{\beta}^{+}$, where $\nu_{2}=\left\langle\alpha, \sigma_{1} \cup\left\{e_{\alpha}\right\}, \tau_{1}\right\rangle$, by the same reasoning in Claim 1a. But if $\nu_{2} \in \mathcal{F}_{\beta}^{+}$, then $\nu_{1} \in \mathcal{F}_{\beta}^{+}$by the Sublemma. In either case $\nu_{1} \in \mathcal{F}_{\beta}^{+}=\mathcal{M}_{\alpha}$, so $\widehat{\nu}_{1} \in \widehat{\mathcal{M}}_{\alpha}$.

Claim 2b. If $\widehat{x} \in \widehat{Y}_{\alpha, s}, \widehat{\nu}_{1}=\widehat{\nu}(\alpha, \widehat{x}, s) \in \mathcal{M}_{\alpha}, s>v_{\alpha}$ of Lemma 8.5(iv), and Red causes enumeration of $\widehat{x}$ so that $\widehat{\nu}_{2}=\widehat{\nu}(\alpha, \widehat{x}, s+1)$, then $\widehat{\nu}_{2} \in \widehat{\mathcal{M}}_{\alpha}$.

Proof. Suppose this enumeration occurs. Then $\widehat{\nu}_{1}<_{R} \widehat{\nu}_{2}$, so $\nu_{1}<_{B} \nu_{2}$ by (37). Now $\widehat{\nu}_{1} \in \widehat{\mathcal{M}}_{\alpha}$, so $\nu_{1} \in \mathcal{M}_{\alpha}$. But $\alpha$ is $\mathcal{M}$-consistent by Lemma 8.11, so $\nu_{2} \in \mathcal{M}_{\alpha}$, and hence $\widehat{\nu}_{2} \in \widehat{\mathcal{M}}_{\alpha}$.

Claim 2c. If $\widehat{x} \in \widehat{Y}_{\alpha, s}, \widehat{\nu}_{1}=(\alpha, \widehat{x}, s) \in \widehat{\mathcal{M}}_{\alpha}, s>v_{\alpha}$ of Lemma 8.5(iv), and Blue causes enumeration of $\widehat{x}$ so that $\widehat{\nu}_{2}=\widehat{\nu}(\alpha, \widehat{x}, s+1)$, then $\widehat{\nu}_{2} \in \widehat{\mathcal{M}}_{\alpha}$.

Proof. Suppose $\widehat{x} \in \widehat{Y}_{\alpha, s}$ and Blue causes this enumeration at stage $s+1$, so $\widehat{\nu}_{1}<_{B} \widehat{\nu}_{2}$. Since $s>v_{\alpha}, \widehat{x} \in \widehat{R}_{\alpha, s} \cap \widehat{R}_{\alpha, s+1}$. Hence, Step $\widehat{1}$, Step $\widehat{3}$, or Step $\widehat{5}$ applies to $\widehat{x}$ at stage $s+1$ for some $\gamma \succeq \alpha$. If Step $\widehat{1}_{\gamma}$ or Step $\widehat{5}_{\gamma}$ applies, then $\widehat{\nu}_{3}=\widehat{\nu}(\gamma, \widehat{x}, s+1) \in \widehat{\mathcal{M}}_{\gamma}$, so $\widehat{\nu}_{2}=\widehat{\nu}_{3} \mid \alpha \in \widehat{\mathcal{M}}_{\alpha}$. (Here Step $\widehat{5}_{\gamma}$ means Step $\widehat{5}$, Case 1 for $\widehat{x} \in \widehat{Y}_{\gamma, s}$ or Step $\widehat{5}$, Case 2 for $\widehat{x} \in \widehat{Y}_{\delta, s}$ where $\gamma=\delta^{-}$.) If Step $\widehat{3}_{\gamma}$ applies, then $\gamma \nsucceq \alpha$ (since $\alpha$ is $\mathcal{M}$-consistent and $\gamma$ is not) and $\widehat{\nu}_{3}=\widehat{\nu}\left(\gamma^{-}, \widehat{x}, s+1\right) \in \widehat{\mathcal{M}}_{\gamma^{-}}$by (3.4), so $\widehat{\nu}_{2}=\widehat{\nu}_{3}\left\lceil\alpha \in \widehat{\mathcal{M}}_{\alpha}\right.$. This completes the proof of Claim 2c.

Claims 2a, 2b, and 2c establish Claim 2 because Claim 2a shows that any entry $\alpha$-state is in $\widehat{\mathcal{M}}_{\alpha}$, and any $\alpha$-state that is well-visited by elements coming from states in $\widehat{\mathcal{M}}_{\alpha}$ must also be in $\widehat{\mathcal{M}}_{\alpha}$. So $\widehat{\mathcal{F}}_{\alpha} \subseteq \widehat{\mathcal{M}}_{\alpha}$. 
Lemma 8.13. $\alpha \subset f \Longrightarrow \alpha$ is $\mathcal{R}$-consistent.

Proof. Assume for a contradiction that $\alpha \prec f$ and $\alpha$ is not $\mathcal{R}$-consistent. Now $\alpha$ is either of length $3 \bmod 5$ or length $4 \bmod 5$. Assume the former, so the $\mathcal{R}$ inconsistency occurs on the $A$-side of the construction. Choose $\nu_{e} \in \mathcal{R}_{\alpha}$ such that for all $\nu \in \mathcal{M}_{\alpha}, \nu_{e} \nless_{R} \nu$. By (66) $\alpha$ is a terminal node on $T$, so $S_{\alpha}=R_{\alpha}$. By Lemmas 8.10 and 8.5(iv), $S_{\alpha, \infty}={ }^{*} \omega$ and no $x \in S_{\alpha, s}, s>v_{\alpha}$, later leaves $S_{\alpha}$. Now $\nu_{e} \in \mathcal{R}_{\alpha} \subseteq \mathcal{M}_{\alpha}=\mathcal{E}_{\alpha}$ by Lemma 8.12, so

$$
\left(\exists^{\infty} x\right)\left(\exists s>v_{\alpha}\right)\left[x \in S_{\alpha, s+1}-Y_{\alpha, s} \quad \& \quad \nu(\alpha, x, s)=\nu_{e}\right] .
$$

Note that for states associated with $\bar{A}$, infinitely many of these $x$ values are in fact $y_{\langle e, k\rangle}$ values for some $k \in \omega$, and thus stay in $\bar{A}$. For each such $x$ and $s$, since $\alpha$ is a terminal node, neither Step 1 nor Step 2 can apply to $x$ at any stage $t>s+1$. Now Step 3 cannot apply to $x \in S_{\alpha, t}$ because $\alpha$ is $\mathcal{M}$-consistent by Lemma 8.11. Furthermore, Step 5 cannot apply to $x \in S_{\alpha, t}$ while $\nu(\alpha, x, t)=\nu_{e}$ because $\nu_{e} \in \mathcal{R}_{\alpha}$ and $\mathcal{R}_{\alpha} \cap \mathcal{B}_{\alpha}=\emptyset$. But if $\nu(\alpha, x, t)=\nu_{e}$ for all $t \geq s$, then $x$ witnesses that $F\left(\alpha^{-}, \nu_{e}\right)$ fails, so $\nu_{e} \in \mathcal{R}_{\alpha}$ contradicts $\alpha \prec f$. Hence, either $x$ is enumerated in $A$ or Step 4 applies to $x$. There must be infinitely many $x$ such that Step 4 applies since infinitely many elements in $\nu_{e}$ never enter $A$. For the dual case, we get that $B$ cannot empty $\nu_{e}$ because for every element that we enumerate into $B$ from $\nu_{e}$, we leave one element in $\bar{B}$. Hence, for infinitely many $x$, Step 4 applies to $x \in S_{\alpha, t}$ at some stage $t+1>s+1$ such that $\nu_{e}=\nu(\alpha, x, s)=\nu(\alpha, x, t)$, $\nu=\nu(\alpha, x, t+1)$, and $\nu_{e}<_{R} \nu$. Choose $\nu$ such that this happens for infinitely many $x \in S_{\alpha}$. Now $\nu \in \mathcal{F}_{\alpha}$, so $\nu \in \mathcal{M}_{\alpha}$ by Lemma 8.12 .

Lemma 8.14. If $\alpha \prec f$ and $\nu_{1} \in \mathcal{B}_{\alpha}$, then $\left\{x: x \in Y_{\alpha} \& \nu(\alpha, x)=\nu_{1}\right\}={ }^{*} \emptyset$.

Proof. Fix $\alpha \prec f$ and $\nu_{1} \in \mathcal{B}_{\alpha}$. Let $v_{\alpha}$ be as in Lemma 8.5(iv). Assume for a contradiction that $x \in R_{\alpha, s}$ for some $s>v_{\alpha}$ and that for all $t \geq s, \gamma=\alpha(x, t)$, and $\nu_{1}=\nu(\alpha, x, t)$. Now $\gamma \succeq \alpha$ and $\alpha \in T$, so by the condition on $T$ in Definition 6.2 (vi) we have $\nu_{1}^{\prime} \in \mathcal{B}_{\gamma}$ for all $\nu_{1}^{\prime} \in \mathcal{M}_{\gamma}$ such that $\nu_{1}^{\prime}\left\lceil\alpha=\nu_{1}\right.$.

Case 1. $\gamma$ is consistent. Then Step 5, Case 1 applies to $x$ and $\gamma$ at some stage $t+1>s$, so $\nu_{1}^{\prime}=\nu(\gamma, x, t), \nu_{2}^{\prime}=\nu(\gamma, x, t+1), \nu_{1}^{\prime}<_{B} \nu_{2}^{\prime}$, and $\nu_{2}^{\prime} \in \mathcal{M}_{\gamma}-\mathcal{B}_{\gamma}$. Hence, $\nu_{2}=\nu_{2}^{\prime}\left\lceil\alpha \in \mathcal{M}_{\alpha}-\mathcal{B}_{\alpha}\right.$, and $\nu(\alpha, x, t+1)=\nu_{2}>_{B} \nu_{1}$.

Case 2. Otherwise. Then at some stage $t+1>s$, Step 5, Case 2 applies to $x$ and $\delta=\gamma^{-} \succeq \alpha$, so $\nu(\alpha, x, t+1)=\nu_{2}>_{B} \nu_{1}$ as in Case 1 but with $\delta$ in place of $\gamma$.

Lemma 8.15. The correspondence $U_{\alpha} \leftrightarrow \widehat{U}_{\alpha}$ and $\widehat{V}_{\alpha} \leftrightarrow V_{\alpha}, \alpha \prec f$, defines an automorphism of $\mathcal{E}^{*}$.

Proof. Choose $\alpha \prec f$. By Lemmas 8.11 and 8.13, $\alpha$ is $\mathcal{M}$-consistent and $\alpha$ is also $\mathcal{R}$-consistent. Hence, $\alpha$ is consistent by Definition 6.1. Thus, by the Definition 6.3. $f$ is infinite, and hence $\lim _{\alpha \prec f} e_{\alpha}=\infty$.

By Lemma 8.10, $Y_{\alpha}={ }^{*} \omega$; by Lemma 8.12, we have (35), its dual, and (36) (so the well-visited $\alpha$-states on $\omega$ coincide with those on $\widehat{\omega}$ ); and by Lemma 8.14 and its dual, we have (39). It immediately follows that the automorphism requirement (25) is satisfied as remarked in $\$ 5.5$ 
8.4. Showing that $D$ is not low and that $B$ is low. The next lemma is a slight modification of Lemma 3.4 .

Lemma 8.16. For every $j \in \omega$ the strategy of every positive requirement node $\beta \prec f$ has satisfied the requirement,

$$
P_{j}: \quad \widehat{\varphi}_{j} \neq \text { characteristic function of }\left\{x: W_{x} \cap \bar{D} \neq \emptyset\right\},
$$

so that $D$ is not low.

Proof. Fix $\beta=f \uparrow(5 j+4)$. Suppose that $\widehat{\varphi}_{j}$ is the $\Delta_{2}^{0}$ characteristic function of $\left\{i: W_{i} \cap \bar{D} \neq \emptyset\right\}$. Hence, for all $i, \lim _{s} \widetilde{\varphi}_{j, s}(i)$ exists and equals $\widehat{\varphi}_{j}(i)$. (Here $\widehat{\varphi}_{j}$ and $\widetilde{\varphi}_{j, s}(i)$ were defined in 3.1 )

Suppose $\widetilde{\varphi}_{j, s}(g(\beta)) \downarrow=0$ for all $s \geq s_{0}$ for some $s_{0}$. Since $\beta \prec f$ there is some stage $s_{1}>s_{0}$ after which $\beta$ is never P-initialized as in Subcase 3 of the $D$-Module because if $\widehat{\psi}_{\gamma}(x)$ is ever defined for $\gamma<_{L} \beta$ after the true path permanently leaves $\gamma$, all witnesses to the right of $\gamma$ get reset to prevent injury, so $\widehat{\psi}_{\gamma}(x)$ will never be redefined. Also for all $\alpha, \alpha \preceq \beta, \alpha$ guesses correctly at the well-visited states, and by Lemma 8.9, for each $e \in \mathcal{M}_{\alpha}$ and each $k, y_{\langle e, k\rangle}^{s}$ is defined at some stage. Hence, $\beta$ waits for a witness $y=x_{n}^{\beta}$ in (72) to appear at some stage $x>s_{1}$. Then according to Case 3 , Subcase 1 of the $D$-Module, $\beta$ will enumerate $x_{n}^{\beta}$ into $Z_{\beta}=W_{g(\beta)}$ causing $Z_{\beta} \cap \bar{D} \neq \emptyset$. Since $\widetilde{\varphi}_{j, t}(g(\beta))=\widetilde{\varphi}_{j, s}(g(\beta))$ for all $t>s, \beta$ will never enumerate $x_{n}^{\beta}$ into $D$, so $\lim _{s} \widetilde{\varphi}_{j, s}(g(\beta))=0$, but $W_{g(\beta)} \cap \bar{D} \neq \emptyset$, a contradiction.

Next suppose $\lim _{s} \widetilde{\varphi}_{j, s}(g(\beta)) \downarrow=1$ for all $s \geq s_{0}$ for some $s_{0}$. For every $s$ there can be at most one element in $Z_{s}^{\beta}-D_{s}$ and that must be $\Gamma_{s}=x_{n}^{\beta}$ for some $n$. Since $\beta \prec f$ we know every $\xi, \xi^{\wedge} 0 \prec \beta$, will have the $\Pi_{2}$-outcome. Hence, for each such $x_{n}^{\beta}$, there will come a stage by (73) at which $x_{n}^{\beta}$ is enumerated in $D$ under Subcase 2. But no new $\beta$-witness can be appointed after stage $s_{0}$ under Subcase 1. Hence, $\lim _{s} \widetilde{\varphi}_{j, s}(g(\beta)) \downarrow=1$, but $W_{g(\beta)} \cap \bar{D}=\emptyset$, a contradiction.

Lemma 8.17. The set $B$ is low .

Proof. Suppose there exist infinitely many $s$ such that $\Phi_{i}^{B}(i)[s] \downarrow$.

Let $s_{0}$ be the least $s$ such that for all $\langle\alpha, x, e\rangle \leq i,\langle\alpha, x, e\rangle$ has either been removed from the list $\Lambda$ already or will never be removed from the list (this can happen if it is never added to the list, or if it is added but never matched). Since each $\langle\alpha, x, e\rangle$ can enter $\Lambda$ only once, then after stage $s_{0}$, no $y$ will enter $B$ in order to match $\langle\alpha, x, e\rangle$. Let $s>s_{0}$ be some stage with $\Phi_{i}^{B}(i)[s] \downarrow$. Then by (B.3), nothing can enter $B$ below the use of this computation. So $(\forall t>s)\left[\Phi_{i}^{B}(i)[t] \downarrow\right]$. So either $\left(\forall^{\infty} s\right)\left[\Phi_{i}^{B}(i)[s] \downarrow\right]$ or $\left(\forall^{\infty} s\right)\left[\Phi_{i}^{B}(i)[s] \uparrow\right]$. Thus, $B$ is low.

We have constructed a set $D$ that is nonlow (by Lemma 8.16) such that for $A=W_{i}=\Psi^{D}$, there is a $B$ that is low (by Lemma 8.17) such that an automorphism of $\mathcal{E}^{*}$ takes $A$ to $B$ (by Lemma 8.15). By [15, page 343], building an automorphism of $\mathcal{E}^{*}$ is equivalent to building one of $\mathcal{E}$. By 4.7 , every c.e. $A \leq_{\mathrm{T}} D$ can be taken to a low set $B$ by an automorphism of $\mathcal{E}$. In particular, every c.e. set $A$ of the same degree as $D$ is automorphic to a low set $B$. Thus, the nonlow degrees cannot be invariant, and so cannot be definable. This completes the proof of Theorem 1.3 , The nonlow degrees are the only upward closed jump class that is not definable. 


\section{REFERENCES}

[1] P. A. Cholak, Automorphisms of the Lattice of Recursively Enumerable Sets, Mem. Amer. Math. Soc. 113 (1995). MR1227497 (95f:03064)

[2] P. A. Cholak and L. A. Harrington, On the Definability of the Double Jump in the Computably Enumerable Sets, J. Math. Log. 2 (2002), 261-296. MR1938925 (2003h:03063)

[3] L. Harrington and R. I. Soare, Definability, automorphisms, and dynamic properties of computably enumerable sets, Bull. Symbolic Logic, 2 (1996), 199-213. MR.1396855 (97d:03057)

[4] L. Harrington and R. I. Soare, The $\Delta_{3}^{0}$ automorphism method and noninvariant classes of degrees, J. Amer. Math. Soc., 9 (1996), 617-666. MR.1311821 (96j:03060)

[5] L. Harrington and R. I. Soare, Definable properties of the computably enumerable sets, Proceedings of the Oberwolfach Conference on Computability Theory, Ann. Pure Appl. Logic, 94 (1998), 97-125. MR1640265 (99f:03055)

[6] A. H. Lachlan, Degrees of recursively enumerable sets which have no maximal superset, $J$. Symbolic Logic 33 (1968), 431-443. MR0236016 (38:4314)

[7] A. H. Lachlan, On some games which are relevant to the theory of recursively enumerable sets, Ann. of Math. (2) 91 (1970), 291-310. MR0284333(44:1562)

[8] D. A. Martin, Classes of recursively enumerable sets and degrees of unsolvability, Z. Math. Logik Grundlagen Math., 12 (1966), 295-310. MR0224469 (37:68)

[9] A. Nies, R. A. Shore, and T. A. Slaman. Definability in the recursively enumerable degrees. Bull. Symbolic Logic 2 (1996), 392-404. MR1460314 (98g:03105)

[10] S. L. Post, Recursively enumerable sets of positive integers and their decision problems, Bull. Amer. Math. Soc., 50 (1944), 284-316. Reprinted in Davis (1965), 304-337. MR0010514 $(6: 29 f)$

[11] J. R. Shoenfield, Degrees of classes of r.e. sets, J. Symbolic Logic 41 (1976), 695-696. MR0485293(58:5140)

[12] G. E. Sacks, Recursive enumerability and the jump operator, Trans. Amer. Math. Soc. 108 (1963), 223-239. MR0155747 (27:5681)

[13] R. I. Soare, Automorphisms of the lattice of recursively enumerable sets, Part I: Maximal sets, Ann. of Math. (2) 100 (1974), 80-120. MR0360235 (50:12685)

[14] R. I. Soare, Automorphisms of the lattice of recursively enumerable sets, Part II: Low sets, Ann. Math. Logic 22 (1982), 69-107. MR661478 (83k:03048)

[15] R. I. Soare, Recursively Enumerable Sets and Degrees: A Study of Computable Functions and Computably Generated Sets, Springer-Verlag, Heidelberg, 1987. MR882921(88m:03003)

Department of Mathematics, Faculty of Arts and Sciences, Harvard University, 1 Oxford Street, Cambridge, Massachusetts 02138

E-mail address: repstein@math.harvard.edu 